\title{
A Geometric Theory of Nonlinear Morphoelastic Shells*
}

\author{
Souhayl Sadik ${ }^{1}$, Arzhang Angoshtari ${ }^{2}$, Alain Goriely ${ }^{3}$, and Arash Yavari $^{\dagger 1,4}$ \\ ${ }^{1}$ School of Civil and Environmental Engineering, Georgia Institute of Technology, Atlanta, GA, USA \\ ${ }^{2}$ Department of Civil and Environmental Engineering, The George Washington University, Washington, DC, USA \\ ${ }^{3}$ Mathematical Institute, University of Oxford, Oxford, UK \\ ${ }^{4}$ The George W. Woodruff School of Mechanical Engineering, Georgia Institute of Technology, Atlanta, GA, USA
}

March 25, 2016

\begin{abstract}
Many thin three-dimensional elastic bodies can be reduced to elastic shells: two-dimensional elastic bodies whose reference shape is not necessarily flat. More generally, morphoelastic shells are elastic shells that can remodel and grow in time. These idealized objects are suitable models for many physical, engineering, and biological systems. Here, we formulate a general geometric theory of nonlinear morphoelastic shells that describes both the evolution of the body shape, viewed as an orientable surface, as well as its intrinsic material properties such as its reference curvatures. In this geometric theory, bulk growth is modeled using an evolving referential configuration for the shell, the so-called material manifold. Geometric quantities attached to the surface, such as the first and second fundamental forms are obtained from the metric of the three-dimensional body and its evolution. The governing dynamical equations for the the body are obtained from variational consideration by assuming that both fundamental forms on the material manifold are dynamical variables in a Lagrangian field theory. In the case where growth can be modeled by a Rayleigh potential, we also obtain the governing equations for growth in the form of kinetic equations coupling the evolution of the first and the second fundamental forms with the state of stress of the shell. We apply these ideas to obtain stress-free growth fields of a planar sheet, the time-evolution of a morphoelastic circular cylindrical shell subject to time-dependent internal pressure, and the residual stress of a morphoelastic planar circular shell.
\end{abstract}

\section{Contents}

1 Introduction 2

2 Differential geometry of shells 3

2.1 Geometry of an embedded surface . . . . . . . . . . . . . . . . . . . . . . . 3

2.2 Idealization of a thin body . . . . . . . . . . . . . . . . . . . . . . . 4

2.3 Evolving geometry of a morphoelastic shell . . . . . . . . . . . . . . . . . . . 6

3 Kinematics of Shells 7

3.1 Strain measures . . . . . . . . . . . . . . . . . . . . . . . . . . 8

3.2 Compatibility equations of shells . . . . . . . . . . . . . . . . . . . . . 9

3.3 Stress-free shell growth . . . . . . . . . . . . . . . . . . . . . . . . . . . . 9

3.4 Velocity and acceleration . . . . . . . . . . . . . . . . . . . . . . . 10

4 The governing equations of motion 11

4.1 Balance of mass . . . . . . . . . . . . . . . . . . . . . . . . . . . 12

4.2 Balance laws . . . . . . . . . . . . . . . . . . . . . . . . . . . . . 13

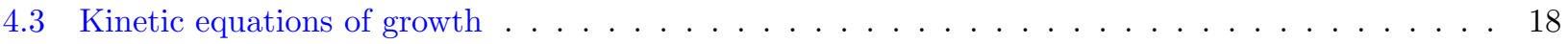

${ }^{*}$ To appear in the Journal of Nonlinear Science.

${ }^{\dagger}$ Corresponding author, e-mail: arash.yavari@ce.gatech.edu 
5 Examples $\quad \mathbf{2 0}$

5.1 Stress-free growth fields for an initially flat simply-connected shell . . . . . . . . . . . . . . 20

5.2 An infinitely long morphoelastic circular cylindrical shell . . . . . . . . . . . . . . . . . . 24

5.3 A morphoelastic circular shell . . . . . . . . . . . . . . . . . . . . . . . . 28

6 Concluding Remarks

\section{Introduction}

Growth and remodeling are particularly important processes in many physical and biological systems [Hori et al., 1986; Silberberg et al., 1989; Pollack et al., 1996; Delsanto et al., 2004; Geitmann and Ortega, 2009], and their interplay with mechanical stress is a well established fact [Hsu, 1968; Skalak, 1982; Skalak et al., 1982; Fung, 1983, 1991, 1995; Taber, 1995; Helmlinger et al., 1997; Humphrey, 2002]. Growth and remodeling of a body can happen in such a way (non-uniformly) that a relaxed state may not exist in the physical space, and since the body is constrained to deform in the Euclidean space, this leads to a state of residual stresses. Such stresses are in fact residual as they persist even when all the external loads are removed [Skalak et al., 1996; Takamizawa and Matsuda, 1990]. Note that the presence of residual stresses in certain biological tissues has been experimentally verified [Chuong and Fung, 1986; Omens and Fung, 1990; Han and Fung, 1991]. Also, as highlighted by Fung [1991], residual stresses are of crucial importance to the working conditions and physiological functions of living organs.

In continuum mechanics, stress is related to a measure of strain, e.g. the deformation gradient, with respect to a stress-free reference configuration. However, in some cases, such a configuration may not be realized in the Euclidean three-dimensional space, i.e., the material manifold is not necessarily Euclidean. This issue has been traditionally addressed by assuming a decomposition of the deformation gradient $\boldsymbol{F}=\boldsymbol{F}_{e} \boldsymbol{F}_{p}$ into an elastic part $\boldsymbol{F}_{e}$ and a non-elastic part $\boldsymbol{F}_{p}$, thus, performing a conceptual local release of stress to a locally stress-free intermediate configuration followed by an elastic deformation to a configuration in the physical space where residual stresses occur [Eckart, 1948; Kröner, 1959; Stojanović et al., 1964]. In the context of growth mechanics, most of the existing formulations rely on this multiplicative decomposition. The non-elastic part of the deformation gradient $\boldsymbol{F}_{p}=\boldsymbol{F}_{g}$ characterizes the growth of the reference configuration and transforms it to a locally relaxed intermediate configuration [Kondaurov and Nikitin, 1987; Rodriguez et al., 1994; Lubarda and Hoger, 2002; Ben Amar and Goriely, 2005]. See [Lubarda, 2004; Ambrosi et al., 2011] for an extensive review and a comprehensive bibliography on the subject and Sadik and Yavari [2016] for a historical perspective on the decomposition of deformation gradient in anelasticity. Recently, Yavari [2010] introduced a geometric theory of growing nonlinear elastic solids in which the stress-free material configuration is a Riemannian manifold with an evolving geometry. This geometrical approach provides a mathematically precise framework to study bulk growth and the induced residual stresses, and leads to a systematic method to find the stress-free growth distributions in nonlinear elasticity.

Research interest in elastic shells was mainly triggered by the experimental work of Chladni [1830] on the tones of vibrating plates, which led to several attempts to lay down a theoretical framework to explain his findings. In 1809, the French Académie des sciences sponsored a prize on the theoretical investigation of the vibration of elastic surfaces and it was won by Germain [1821] whose work proved later on to be partially incorrect (see the historical introduction by Love [1892]). The first attempts to formulate a general theory and obtain the governing equations for the deformation of elastic shells are attributed to Aron [1874] and Mathieu [1882]. However, it was Love [1888] who first obtained a consistent general theory for small strains of linear elastic shells based on the work of Kirchhoff [1850] on the vibrations of plates.

The governing equations of elastic shells in terms of stress and couple stress tensors were first derived in rectangular Cartesian coordinates by Cosserat and Cosserat [1909] (see [Ericksen and Truesdell, 1958] for an extension of Cosserats' work). The coordinate-free expression of these equations was, however, presented in its full generality by Synge and Chien [1941]. They localized the integral balance laws of shells valid in a Cartesian coordinate chart to obtain the governing differential equations and then obtained the coordinate-free expressions of these differential equations. Alternative derivations for the governing equations of elastic shells in terms of stress and couple stress tensors were later proposed by Novozhilov [1964]; Green and Zerna [1950]; Naghdi [1963] starting from the general three-dimensional equations of equilibrium and by [Koiter, 1966] by means of the principle of virtual work. 
While considerable progress has been made in the modeling of three-dimensional growing elastic bodies,a complete continuum theory for morphoelastic shells is not yet available. In particular, there is no general fomulation for the computation of residual stresses and couple stresses due to bulk growth in shells. All the aforementioned shell theories are restricted to model shells in the context of mass-conserving elasticity. It is worthwhile, however, to mention the work by Goriely and Ben Amar [2005] on a growing shell embedded in an elastic medium and the ongoing effort on modeling morphoelastic plates [Efrati et al., 2009; Dervaux et al., 2009; McMahon et al., 2011a,b].

More recently, Pezzulla et al. [2015a,b] investigated geometry-driven growth-like morphing of thin bilayer shells. Remarkably, they were able to predict and experimentally obtain domes or a saddle surface in a shrink-fit problem in thin circular disks [Pezzulla et al., 2015a]. They also showed that a large isotropic expansion of one layer with respect to the other leads to a cylindrical bending of the bilayer sheet [Pezzulla et al., 2015b].

In this paper, following Yavari [2010]'s approach of an evolving material manifold to model bulk growth, we develop a geometric nonlinear theory of morphoelastic shells. We model bulk growth in orientable surfaces using evolving first and second fundamental forms in the material manifold. In $\S 2$, we discuss the idealization of a thin body to a shell and its evolving referential geometry as an embedded hyperplane to model growth through evolving first and second fundamental forms. In $\S 3$, we present the kinematics of shells, include a discussion on the compatibility equations, and introduce a systematic method to find those growth fields that leave a stressfree shell stress-free. In $\S 4$, we derive the balance of mass for morphoelastic shells. Following a Lagrangian field theory, we also derive the balance laws for morphoelastic shells and the kinetic equations for the evolution of growth such that the evolution of the first and the second fundamental forms is coupled with the state of stress of the shell. In $\S 5$ we look at a few examples to demonstrate the capability of the proposed geometric theory in calculation of the time evolution of residual stresses induced by growth. First, we consider a planar shell and look for a family of growth fields that leave the shell stress-free. We numerically find embeddings of the evolving surfaces in $\mathbb{R}^{3}$. We find that these stress-free growth distributions of the initially planar shell can force it to evolve to either another flat sheet (e.g. cylindrical sheets), sheets with positive curvature (e.g. spherical sheets), or sheets with negative curvature (e.g. saddle-like sheets). Next we look at the problems of a morphoelastic infinitely long circular cylindrical shell subject to a time-dependent internal pressure and that of a morphoelastic initially planar circular disk. We numerically solve the kinetic equations for the evolution of growth and observe the coupling between the state of stress of the shell and the evolution of its curvature. We consequently obtain the evolution of the geometry of the shells and the induced residual stress and couple-stress fields.

\section{Differential geometry of shells}

In this section, we review a few elements of the differential geometry of shells as embedded surfaces in threedimensional manifolds, discuss the idealization of a thin body as a shell, and the evolving geometry of a morphoelastic shell.

\subsection{Geometry of an embedded surface}

In this section, we tersely review some elements of the geometry of two-dimensional embedded surfaces in threedimensional manifolds (see for example [Hicks, 1965; do Carmo, 1992] for a detailed account on the subject). Let $(\mathcal{B}, \bar{G})$ be an orientable three-dimensional Riemannian manifold and let $(\mathcal{H}, \boldsymbol{G})$ be an orientable two-dimensional Riemannian submanifold of $(\mathcal{B}, \bar{G})$, i.e., $\boldsymbol{G}=\left.\overline{\boldsymbol{G}}\right|_{\mathcal{H}}$. Let $\mathfrak{X}(\mathcal{H})$ be the space of smooth tangent vector fields on $\mathcal{H}$. Using the decomposition $T_{X} \mathcal{B}=T_{X} \mathcal{H} \oplus\left(T_{X} \mathcal{H}\right)^{\perp}, \forall X \in \mathcal{H}$, we define the space of smooth normal vector fields $\mathfrak{X}(\mathcal{H})^{\perp} \subset \mathfrak{X}(\mathcal{B})$. Let $\boldsymbol{N} \in \mathfrak{X}(\mathcal{H})^{\perp}$ be the smooth unit normal vector field of $\mathcal{H}$. The orientation of the unit normal vector field $\boldsymbol{N}$ is chosen such that the orientation induced by the local coordinate chart of the surface $\mathcal{H}$ and the unit normal vector field as the last coordinate on $\mathcal{B}$ is consistent with the orientation of $\mathcal{B}$. Let $\nabla^{\mathcal{H}}$ and $\bar{\nabla}$ be the Levi-Civita connections of $(\mathcal{H}, \boldsymbol{G})$ and $(\mathcal{B}, \bar{G})$, respectively. Note that the Levi-Civita connection $\nabla^{\mathcal{H}}$ of the metric $\boldsymbol{G}$ is precisely the connection induced by the Levi-Civita connection $\bar{\nabla}$ of the metric $\overline{\boldsymbol{G}}$. The connection $\nabla^{\mathcal{H}}$ in terms of the connection $\bar{\nabla}$ is given by

$$
\nabla_{\boldsymbol{X}}^{\mathcal{H}} \boldsymbol{Y}=\bar{\nabla}_{\bar{X}} \overline{\boldsymbol{Y}}-\overline{\boldsymbol{G}}\left(\bar{\nabla}_{\overline{\mathbf{X}}} \overline{\boldsymbol{Y}}, \boldsymbol{N}\right) \boldsymbol{N}, \forall \boldsymbol{X}, \boldsymbol{Y} \in \mathfrak{X}(\mathcal{H}),
$$


where $\overline{\boldsymbol{X}} \in \mathfrak{X}(\mathcal{B})$ and $\overline{\boldsymbol{Y}} \in \mathfrak{X}(\mathcal{B})$ are any local extensions of $\boldsymbol{X}$ and $\boldsymbol{Y}$, respectively, i.e., $\overline{\boldsymbol{X}}(X)=\boldsymbol{X}(X)$, $\forall X \in \mathcal{H}$. The second fundamental form of $\mathcal{H}$ is defined as the symmetric tensor $\boldsymbol{B} \in \Gamma\left(S^{2} T^{*} \mathcal{H}\right)$ given by

$$
\boldsymbol{B}(\boldsymbol{X}, \boldsymbol{Y})=\overline{\boldsymbol{G}}\left(\bar{\nabla}_{\overline{\boldsymbol{X}}} \overline{\boldsymbol{Y}}, \boldsymbol{N}\right)=-\overline{\boldsymbol{G}}\left(\bar{\nabla}_{\overline{\boldsymbol{X}}} \boldsymbol{N}, \overline{\boldsymbol{Y}}\right), \forall \boldsymbol{X}, \boldsymbol{Y} \in \mathfrak{X}(\mathcal{H}) .
$$

The connection $\nabla$ on $T \mathcal{H}$ induces a connection on $S^{2} T^{*} \mathcal{H}$ defined by

$$
\left(\nabla_{\boldsymbol{X}} \boldsymbol{A}\right)(\boldsymbol{Y}, \boldsymbol{Z})=\boldsymbol{X}(\boldsymbol{A}(\boldsymbol{Y}, \boldsymbol{Z}))-\boldsymbol{A}\left(\nabla_{\boldsymbol{X}} \boldsymbol{Y}, \boldsymbol{Z}\right)-\boldsymbol{A}\left(\boldsymbol{Y}, \nabla_{\boldsymbol{X}} \boldsymbol{Z}\right), \quad \forall \boldsymbol{A} \in \Gamma\left(S^{2} T^{*} \mathcal{H}\right) .
$$

The curvature tensor $\mathcal{R}$ of a Riemannian manifold $(M, \boldsymbol{G})$ is defined as

$$
\mathcal{R}(\boldsymbol{X}, \boldsymbol{Y}, \boldsymbol{Z}, \boldsymbol{W})=\boldsymbol{G}(\boldsymbol{R}(\boldsymbol{X}, \boldsymbol{Y}) \boldsymbol{Z}, \boldsymbol{W}), \forall \boldsymbol{X}, \boldsymbol{Y}, \boldsymbol{Z}, \boldsymbol{W} \in \mathfrak{X}(\mathcal{M}),
$$

where $\boldsymbol{R}$ is given in terms of the Levi-Civita connection $\nabla^{M}$ by

$$
\boldsymbol{R}(\boldsymbol{X}, \boldsymbol{Y}) \boldsymbol{Z}=\nabla_{[\boldsymbol{X}, \boldsymbol{Y}]}^{M} \boldsymbol{Z}-\nabla_{\boldsymbol{X}}^{M} \nabla_{\boldsymbol{Y}}^{M} \boldsymbol{Z}+\nabla_{\boldsymbol{Y}}^{M} \nabla_{\boldsymbol{X}}^{M} \boldsymbol{Z} .
$$

In components, the curvature tensor reads

$$
\mathcal{R}_{A B C D}=\mathcal{R}\left(\partial_{A}, \partial_{B}, \partial_{C}, \partial_{D}\right)=\left(\partial_{B} \Gamma^{K}{ }_{A C}-\partial_{A} \Gamma^{K}{ }_{B C}+\Gamma^{L}{ }_{A C} \Gamma^{K}{ }_{B L}-\Gamma^{L}{ }_{B C} \Gamma^{K}{ }_{A L}\right) G_{K D} .
$$

Given the symmetries of the curvature tensor, if $n$ is the dimension of the manifold $M$, its curvature tensor $\mathcal{R}$ has $n^{2}\left(n^{2}-1\right) / 12$ independent components. In particular, for a two-dimensional surface $(n=2)$, the curvature tensor has one independent component $\mathcal{R}_{1221}$.

We denote the Riemann curvature tensors of $\mathcal{H}$ and $\mathcal{B}$ by $\mathcal{R}^{\mathcal{H}}$ and $\overline{\mathcal{R}}$, respectively. The Gauss equation gives a relation between the Riemann curvature tensor and the second fundamental form of $\mathcal{H}$, and the Riemann curvature tensor of $\mathcal{B}$ as

$$
\overline{\mathcal{R}}(\boldsymbol{X}, \boldsymbol{Y}, \boldsymbol{Z}, \boldsymbol{W})=\mathcal{R}^{\mathcal{H}}(\boldsymbol{X}, \boldsymbol{Y}, \boldsymbol{Z}, \boldsymbol{W})-\boldsymbol{B}(\boldsymbol{X}, \boldsymbol{Z}) \boldsymbol{B}(\boldsymbol{Y}, \boldsymbol{W})+\boldsymbol{B}(\boldsymbol{X}, \boldsymbol{W}) \boldsymbol{B}(\boldsymbol{Y}, \boldsymbol{Z}) .
$$

The second fundamental form also satisfies the Codazzi-Mainardi equation that can be written as

$$
\overline{\mathcal{R}}(\boldsymbol{X}, \boldsymbol{Y}, \boldsymbol{Z}, \boldsymbol{N})=\left(\nabla_{\boldsymbol{Y}}^{\mathcal{H}} \boldsymbol{B}\right)(\boldsymbol{X}, \boldsymbol{Z})-\left(\nabla_{\boldsymbol{X}}^{\mathcal{H}} \boldsymbol{B}\right)(\boldsymbol{Y}, \boldsymbol{Z}) .
$$

Let $\left(X^{1}, X^{2}, X^{3}\right)$ be a local coordinate chart for $\mathcal{B}$ such that at any point of the hypersurface $\mathcal{H},\left\{X^{1}, X^{2}\right\}$ is a local coordinate chart for $\mathcal{H}$ and the normal vector field $\boldsymbol{N}$ to $\mathcal{H}$ is tangent to the coordinate curve $X^{3}$. We say that such a chart is compatible with $\mathcal{H}$. Note that given the symmetries of the curvature tensor and the second fundamental form, the Gauss and Codazzi-Mainardi equations reduce in components to

$$
\begin{aligned}
\overline{\mathcal{R}}_{1212}-\mathcal{R}_{1212}^{\mathcal{H}} & =B_{11} B_{22}-B_{12} B_{12}, \\
\overline{\mathcal{R}}_{1213} & =B_{11 \mid 2}-B_{21 \mid 1}, \\
\overline{\mathcal{R}}_{2123} & =B_{22 \mid 1}-B_{12 \mid 2},
\end{aligned}
$$

where we denote by a stroke $\mid$ the covariant derivative corresponding to the Levi-Civita connection of $(\mathcal{H}, \boldsymbol{G})$, i.e., $B_{A B \mid C}=B_{A B, C}-\Gamma^{K}{ }_{C A} B_{K B}-\Gamma^{K}{ }_{C B} B_{A K}$, where $\Gamma^{C}{ }_{A B}$ is the Christoffel symbol of the connection $\nabla^{\mathcal{H}}$ in the local chart $\left\{X^{1}, X^{2}\right\}$.

The fundamental theorem of surface theory, first proved by Bonnet [1865], implies that the geometry of a surface is fully described by its metric and its second fundamental form [do Carmo, 1976; Ivey and Landsberg, 2003].

\subsection{Idealization of a thin body}

Let $B$ be a three-dimensional thin body (i.e. its thickness is negligible compared to the other two dimensions) identified with an orientable three-dimensional Riemannian manifold $\mathcal{B}$ endowed with the metric $\overline{\boldsymbol{G}}$. Let $H$ - the mid-surface of $B$-be identified with $(\mathcal{H}, \boldsymbol{G}, \boldsymbol{B})$, a two-dimensional Riemannian submanifold of $(\mathcal{B}, \overline{\boldsymbol{G}})$ with first and second fundamental forms $\boldsymbol{G}$ and $\boldsymbol{B}$ (see Figure 1). We assume in the following that $\mathcal{H}$ is an orientable hypersurface of $\mathcal{B}$. We show that the natural isometric embedding of $\mathcal{H}$ in $\mathcal{B}$ induces independent in-plane and out-of-plane geometries for the hypersurface $\mathcal{H}$. 


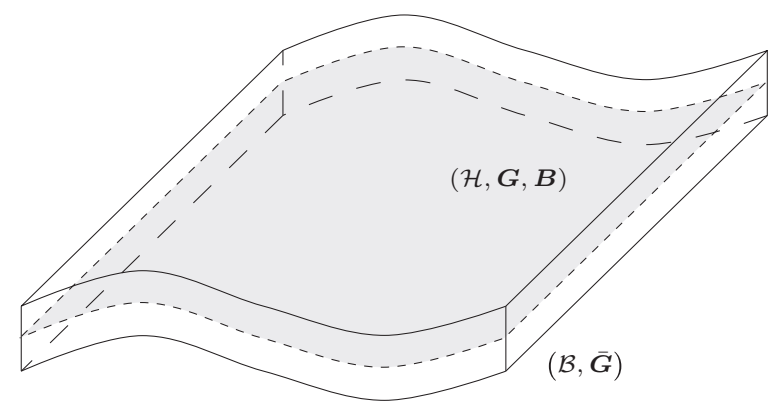

Figure 1: The mid-surface $(\mathcal{H}, \boldsymbol{G})$ modeled as a Riemannian submanifold of $(\mathcal{B}, \overline{\boldsymbol{G}})$.

Let $\left(X^{1}, X^{2}, X^{3}\right)$ be a local coordinate chart compatible with $\mathcal{H}$. In this coordinate chart, at any point $X \in \mathcal{B}$, the metric $\overline{\boldsymbol{G}}$ of $\mathcal{B}$ has the following representation:

$$
\overline{\boldsymbol{G}}(X)=\left(\begin{array}{ccc}
\bar{G}_{11}(X) & \bar{G}_{12}(X) & \bar{G}_{13}(X) \\
\bar{G}_{12}(X) & \bar{G}_{22}(X) & \bar{G}_{23}(X) \\
\bar{G}_{13}(X) & \bar{G}_{23}(X) & \bar{G}_{33}(X)
\end{array}\right)
$$

If $X \in \mathcal{H}$, we have

$$
\overline{\boldsymbol{G}}(X)=\left(\begin{array}{ccc}
\bar{G}_{11}(X) & \bar{G}_{12}(X) & 0 \\
\bar{G}_{12}(X) & \bar{G}_{22}(X) & 0 \\
0 & 0 & 1
\end{array}\right) .
$$

Thus, the metric $\boldsymbol{G}$ of $\mathcal{H}$, referred to as the first fundamental form, has the following representation

$$
\boldsymbol{G}(X)=\left(\begin{array}{ll}
\bar{G}_{11}(X) & \bar{G}_{12}(X) \\
\bar{G}_{12}(X) & \bar{G}_{22}(X)
\end{array}\right), \forall X \in \mathcal{H}
$$

and the second fundamental form of $\mathcal{H}$ has the following components

$$
B_{A B}(X)=\bar{\Gamma}_{A B}^{3}(X), A, B=1,2, \forall X \in \mathcal{H},
$$

where $\bar{\Gamma}_{A B}^{C}=\frac{1}{2} \sum_{K} \bar{G}^{C K}\left(\partial_{A} \bar{G}_{K B}+\partial_{B} \bar{G}_{K A}-\partial_{K} \bar{G}_{A B}\right)$ is the Christoffel symbol of the Levi-Civita connection of $(\mathcal{B}, \bar{G})$. Therefore

$$
B_{A B}(X)=-\left.\frac{1}{2} \frac{\partial \bar{G}_{A B}}{\partial X^{3}}\right|_{\mathcal{H}}(X), A, B=1,2, \forall X \in \mathcal{H}
$$

where $\bar{G}_{A B}$ should be thought of as a function on the coordinate curve $X^{3}$ and $\left.\frac{\partial \bar{G}_{A B}}{\partial X^{3}}\right|_{\mathcal{H}}$ is evaluated at the point where the curve $X^{3}$ meets the hypersurface $\mathcal{H}$. Since $\bar{G}_{A B}$ and $\frac{\partial \bar{G}_{A B}}{\partial X^{3}}$ can be prescribed independently, equations (2.5) and (2.6) demonstrate that independent first and second fundamental forms $\mathbf{G}$ and $\mathbf{B}$ of the hypersurface $\mathcal{H}$ can be obtained from the metric $\overline{\boldsymbol{G}}$ of the embedding space $\mathcal{B}$. Therefore, we only need to specify the components $\bar{G}_{A B}$ for $A, B=1,2$ to characterize the geometry of $\mathcal{H}$. We introduce the following notation

$$
\overline{\boldsymbol{G}}_{\mathcal{H}}(X):=\left(\begin{array}{ll}
\bar{G}_{11}(X) & \bar{G}_{12}(X) \\
\bar{G}_{12}(X) & \bar{G}_{22}(X)
\end{array}\right), \forall X \in \mathcal{B}
$$

Remark 2.1. In the local coordinate chart $\left(X^{1}, X^{2}, X^{3}\right)$, note that the components $\bar{G}_{A 3}, A=1,2,3$ of the metric $\overline{\boldsymbol{G}}$ do not affect the geometry of $\mathcal{H}$. Indeed, from equations (2.5) and (2.6), the geometry of $\mathcal{H}$ depends only on the restriction of the metric $\overline{\boldsymbol{G}}$ to $\mathcal{H}$ (i.e., $\left.\left.\bar{G}_{A B}\right|_{\mathcal{H}}, A, B=1,2\right)$ and its first order derivative along the normal to $\mathcal{H}$ (i.e., $\left.\frac{\partial \bar{G}_{A B}}{\partial X^{3}}\right|_{\mathcal{H}}, A, B=1,2$ ). Therefore, higher order variations of $\overline{\boldsymbol{G}}_{\mathcal{H}}$ along the thickness of $B$ are not captured by the geometry of $\mathcal{H}$. As an example, let $\mathcal{B}$ be a thin body in $\mathbb{R}^{3}$ with the coordinate chart $\left(X^{1}, X^{2}, X^{3}\right)$ such that the hypersurface $X^{3}=0$ contains the mid-surface $\mathcal{H}$. Two different metrics for $\mathcal{B}$ such that $\bar{G}_{A B}^{1}(X)=e^{X^{3}} \delta_{A B}$, and $\bar{G}_{A B}^{2}(X)=\left(1+X^{3}\right) \delta_{A B}, \forall X \in \mathcal{B}, A, B=1,2$, correspond to the same 
geometry for $\mathcal{H}$ given by $G_{A B}=\delta_{A B}$ and $B_{A B}=-\frac{1}{2} \delta_{A B}, A, B=1,2$. Also, if we consider an evolving metric such that $\bar{G}_{A B}(X, t)=\left(1+\left(X^{3}\right)^{2} f(t)\right) \delta_{A B}, \forall X \in \mathcal{B}, A, B=1,2$, where $f$ is a given function of time, we find that the geometry of $\mathcal{H}$ does not capture this evolution as it remains unchanged both in-plane and out-of-plane $(\boldsymbol{G}=\boldsymbol{\delta}$ and $\boldsymbol{B}=\mathbf{0})$.

\subsection{Evolving geometry of a morphoelastic shell}

In order to model bulk growth of the body $\mathcal{B}$, we assume, following Yavari [2010], an evolving metric for the material manifold $(\mathcal{B}, \bar{G})$, i.e., we leave the manifold $\mathcal{B}$ fixed and endow it with an evolving metric ${ }^{1} \overline{\boldsymbol{G}}$, i.e., $\overline{\boldsymbol{G}}=\overline{\boldsymbol{G}}(X, t)$, such that at $t=0$, we have $\overline{\boldsymbol{G}}(X, 0)=\overline{\boldsymbol{G}}^{0}(X)$ the metric of a natural stress-free configuration of $\mathcal{B}$. In this paper, however, we are interested in growth of thin bodies, and hence, we consider the manifold $(\mathcal{B}, \overline{\boldsymbol{G}})$ with an evolving metric such that, in a local coordinate chart $\left(X^{1}, X^{2}, X^{3}\right)$ as introduced in $\S 2.2$, only $\overline{\boldsymbol{G}}_{\mathcal{H}}$ is evolving. ${ }^{2}$ Now, we leave the mid-surface manifold $\mathcal{H}$ fixed and let its evolving first and second fundamental forms $\boldsymbol{G}$ and $\boldsymbol{B}$ be induced from its natural isometric embedding in $\mathcal{B}$. Therefore, in the local coordinate chart $\left(X^{1}, X^{2}, X^{3}\right)$, the metric reads

$$
\boldsymbol{G}(X, t)=\left(\begin{array}{cc}
\bar{G}_{11}(X, t) & \bar{G}_{12}(X, t) \\
\bar{G}_{12}(X, t) & \bar{G}_{22}(X, t)
\end{array}\right), \quad \forall X \in \mathcal{H}
$$

and the second fundamental form of $\mathcal{H}$ is written as

$$
B_{A B}(X, t)=-\left.\frac{1}{2} \frac{\partial \bar{G}_{A B}}{\partial X^{3}}\right|_{\mathcal{H}}(X, t), A, B=1,2, \forall X \in \mathcal{H} .
$$

We will discuss, in $\S 4.2$, the governing equations for the evolution of the first and the second fundamental forms of shells and see how the evolving geometry of the material manifold, i.e., the growth of the morphoelastic shell, is coupled with its current state of stress. Note that the evolving fundamental forms $\boldsymbol{G}$ and $\boldsymbol{B}$ are compatible in the manifold $(\mathcal{B}, \overline{\boldsymbol{G}})$, i.e., they satisfy the Gauss and Codazzi-Mainardi equations (2.2) and (2.3).

To illustrate the evolving geometry of a surface, we consider a flat thin body that can be represented by a planar surface $(\mathcal{H}, \boldsymbol{G})$ in $\left(\mathbb{R}^{3}, \overline{\boldsymbol{G}}\right)$. Let $\left(X^{1}, X^{2}, X^{3}\right)$ be the standard coordinate chart for $\mathbb{R}^{3}$ such that the hyperplane $X^{3}=0$ contains the surface $\mathcal{H}$. If we assume that the body undergoes a growth that is uniform through its thickness, then we can model this growth by an evolving metric $\overline{\boldsymbol{G}}_{\mathcal{H}}$ such that $\bar{G}_{A B}(X, t)=$ $\bar{G}_{A B}\left(X^{1}, X^{2}, t\right), \forall X \in \mathcal{B}, A, B=1,2$ (i.e., $\bar{G}_{A B}$ 's do not depend on $X^{3}$ ), then, we obtain an evolving geometry for $\mathcal{H}$ with an evolving metric $G_{A B}(X, t)=\bar{G}_{A B}(X, t), \forall X \in \mathcal{H}$ and a vanishing second fundamental form. As an example $\bar{G}_{A B}\left(X^{1}, X^{2}, t\right)=f(t) \delta_{A B}, A, B=1,2$, for some function $f$ of time, models a uniform inplane growth with no out-of-plane geometry change (i.e., a vanishing second fundamental form). However, if we assume that the body undergoes a growth that is not uniform through its thickness we obtain an evolving geometry for $\mathcal{H}$ such that the second fundamental form evolves with time: $B_{A B}(X, t)=-\left.\frac{1}{2} \frac{\partial \bar{G}_{A B}}{\partial X^{3}}\right|_{\mathcal{H}}(X, t)$. As an example, we let $\bar{G}_{A B}(X, t)=f\left(X^{3}, t\right) \delta_{A B}, A, B=1,2$, for some function $f$ of time and $X^{3}$ such that $f(0, t)=1, \frac{\partial f}{\partial X^{3}}(0,0)=0$, and $\frac{\partial f}{\partial X^{3}}(0, t) \neq 0$ for $t \neq 0$ (e.g. $f\left(X^{3}, t\right)=e^{X^{3} t}$ ). Then we have an evolving geometry for $\mathcal{H}$ such that the metric of $\mathcal{H}$ remains unchanged: $G_{A B}=\delta_{A B}$ while its out-of-plane geometry evolves with time: $B_{A B}=-\frac{1}{2} \frac{\partial f}{\partial X^{3}}(0, t) \delta_{A B}$.

Remark 2.2. Given the fact (discussed in Remark 2.1) that the geometry of $\mathcal{H}$ depends only on the restriction of the metric $\bar{G}$ to $\mathcal{H}$ and its first order derivative along the normal to $\mathcal{H}$, we are bound to model a restrictive class of material evolutions. We assume that the evolving material manifold $(\mathcal{H}, \boldsymbol{G})$ at time $t$ is diffeomorphic to the reference manifold $\left(\mathcal{H}, G^{0}\right)$ at time $t=0$, so that this diffeomorphism can be extended to a neighborhood of $\mathcal{H}$ in $\mathcal{B}$ in such a way that the push-forward of the reference normal vector field $\boldsymbol{N}^{0}$ of $\mathcal{H}$ in $\left(\mathcal{B}, \bar{G}^{0}\right)$ is precisely the evolving normal vector field $\boldsymbol{N}$ of $\mathcal{H}$ in $\left(\mathcal{B}, \bar{G}^{0}\right)$. Note that this implies that during the material evolution of the shell, at any point of $\mathcal{H}$, the normal to $\mathcal{H}$ remains normal.

We can write the evolving metric $\overline{\boldsymbol{G}}_{\mathcal{H}}$ in the form

$$
\overline{\boldsymbol{G}}_{\mathcal{H}}(X, t)=\overline{\boldsymbol{G}}_{\mathcal{H}}^{0}(X) e^{2 \overline{\boldsymbol{\omega}}(X, t)}, \quad \forall X \in \mathcal{B},
$$

\footnotetext{
${ }^{1}$ Other examples of evolving material metrics in mechanics have been introduced in [Ozakin and Yavari, 2010; Yavari and Goriely, 2012a,b, 2013a, 2015, 2013b, 2014; Sadik and Yavari, 2015].

${ }^{2}$ cf. (2.7) where the notation $\overline{\boldsymbol{G}}_{\mathcal{H}}$ was introduced.
} 
where $\bar{\omega}$ is a smooth $\left(\begin{array}{l}1 \\ 1\end{array}\right)$-rank tensor characterizing growth of the thin body $\mathcal{B}$ such that $\bar{\omega}(X, 0)=\mathbf{0}$. Following (2.8), the evolving first fundamental form of $\mathcal{H}$ is given by

$$
\boldsymbol{G}(X, t)=\overline{\boldsymbol{G}}_{\mathcal{H}}^{0}(X) e^{2 \boldsymbol{\omega}(X, t)}, \quad \forall X \in \mathcal{H},
$$

where $\omega=\left.\bar{\omega}\right|_{\mathcal{H}}$. Following (2.9), for $X \in \mathcal{H}$, the evolving second fundamental form of $\mathcal{H}$ is given by

$$
\begin{aligned}
\boldsymbol{B}(X, t) & =-\frac{1}{2} \frac{\partial \overline{\boldsymbol{G}}_{\mathcal{H}}}{\partial X^{3}}(X, t) \\
& =-\frac{1}{2} \frac{\partial}{\partial X^{3}}\left[\overline{\boldsymbol{G}}_{\mathcal{H}}^{0} e^{2 \overline{\boldsymbol{\omega}}}\right](X, t) \\
& =-\frac{1}{2} \frac{\partial \overline{\boldsymbol{G}}_{\mathcal{H}}^{0}}{\partial X^{3}}(X) e^{2 \overline{\boldsymbol{\omega}}(X, t)}-\overline{\boldsymbol{G}}_{\mathcal{H}}^{0}(X) \frac{\partial \overline{\boldsymbol{\omega}}}{\partial X^{3}}(X, t) e^{2 \overline{\boldsymbol{\omega}}(X, t)} .
\end{aligned}
$$

For $X \in \mathcal{H}$, we introduce the following notations:

$$
\boldsymbol{G}^{0}(X):=\overline{\boldsymbol{G}}_{\mathcal{H}}^{0}(X), \quad \boldsymbol{B}^{0}(X):=-\frac{1}{2} \frac{\partial \overline{\boldsymbol{G}}_{\mathcal{H}}^{0}}{\partial X^{3}}(X), \quad \omega(X, t):=\bar{\omega}(X, t), \text { and } \quad \boldsymbol{K}(X, t)=\frac{\partial \overline{\boldsymbol{\omega}}}{\partial X^{3}}(X, t),
$$

and hence we write the evolving first and second fundamental forms of $\mathcal{H}$ as

$$
\boldsymbol{G}(X, t)=\boldsymbol{G}^{0}(X) e^{2 \boldsymbol{\omega}(X, t)}, \quad \boldsymbol{B}(X, t)=\boldsymbol{B}^{0}(X) e^{2 \boldsymbol{\omega}(X, t)}-\boldsymbol{G}^{0}(X) \boldsymbol{K}(X, t) e^{2 \boldsymbol{\omega}(X, t)},
$$

such that $\boldsymbol{\omega}(X, 0)=\mathbf{0}$ and $\boldsymbol{K}(X, 0)=\mathbf{0}$, so that at $t=0, \boldsymbol{G}(t=0)=\boldsymbol{G}^{0}$ and $\boldsymbol{B}(t=0)=\boldsymbol{B}^{0}$. For isotropic growth, we assume $\omega_{1}=\omega_{2}=\omega$ and $K_{1}=K_{2}=K$, recalling that for $A=\{1,2\}, K_{A}=\frac{\partial \bar{\omega}_{A}}{\partial X^{3}}$. Therefore, we have

$$
\boldsymbol{G}(X, t)=\boldsymbol{G}^{0}(X) e^{2 \omega(X, t)}, \quad \boldsymbol{B}(X, t)=\boldsymbol{B}^{0}(X) e^{2 \omega(X, t)}-K(X, t) \boldsymbol{G}^{0}(X) e^{2 \omega(X, t)},
$$

such that $\omega(X, 0)=0$ and $K(X, 0)=0$.

Remark 2.3. The Riemannian surface form-i.e., the volume form of the surface $\mathcal{H}$-associated with the metric $\boldsymbol{G}$ is written as

$$
d S(X, \boldsymbol{G})=\sqrt{\operatorname{det} \boldsymbol{G}} d X^{1} \wedge d X^{2}=\sqrt{\operatorname{det} \boldsymbol{G}^{0}} e^{\operatorname{tr}(\boldsymbol{\omega}(X, T))} d X^{1} \wedge d X^{2}=e^{\operatorname{tr}(\boldsymbol{\omega}(X, t))} d S_{0}(X),
$$

where $d S_{0}$ is the Riemannian surface form associated with the metric $\boldsymbol{G}^{0}$. Using the identity $\operatorname{det} e^{\boldsymbol{A}}=e^{\operatorname{tr}(\boldsymbol{A})}$, the rate of change of the volume element due to the evolving metric is given by

$$
\frac{d}{d t} d S(X, \boldsymbol{G})=\frac{d}{d t}[\operatorname{tr}(\boldsymbol{\omega}(X, t))] d S(X, \boldsymbol{G}) .
$$

Alternatively, by using the identity $\frac{d}{d t}[\operatorname{det} \boldsymbol{A}(t)]=\operatorname{det} \boldsymbol{A}(t) \operatorname{tr}\left[\boldsymbol{A}^{-1}(t) \frac{d}{d t} \boldsymbol{A}(t)\right]$, we find that

$$
\frac{d}{d t} d S(X, \boldsymbol{G})=\frac{1}{2} \operatorname{tr}\left(\frac{d \boldsymbol{G}}{d t}\right) d S(X, \boldsymbol{G}) .
$$

\section{Kinematics of Shells}

Let the ambient space be $\mathcal{S}=\mathbb{R}^{3}$ endowed with the standard Euclidean metric $\tilde{\boldsymbol{g}}$. Recall that the Riemannian surface $(\mathcal{H}, \boldsymbol{G}, \boldsymbol{B})$ is an orientable two-dimensional Riemannian submanifold of $(\mathcal{B}, \overline{\boldsymbol{G}})$. A configuration of $\mathcal{H}$ in $\mathcal{S}$ is a smooth embedding $\varphi: \mathcal{H} \rightarrow \mathcal{S}$. We denote the set of all configurations of $\mathcal{H}$ in $\mathcal{S}$ by $\mathcal{C}$. As shown in Figure 2, the Riemannian manifold $(\varphi(\mathcal{H}), \boldsymbol{g}, \boldsymbol{\beta})$, where $\boldsymbol{g}:=\left.\tilde{\boldsymbol{g}}\right|_{\varphi(\mathcal{H})}$ and $\boldsymbol{\beta} \in \Gamma\left(S^{2} T^{*} \varphi(\mathcal{H})\right)$ the second fundamental form of $\varphi(\mathcal{H})$, is a hypersurface in $\mathcal{S}$. Let $\nabla$ and $\tilde{\nabla}$ be the Levi-Civita connections of $\boldsymbol{g}$ and $\tilde{\boldsymbol{g}}$, respectively. Let $\boldsymbol{n} \in \mathfrak{X}(\varphi(\mathcal{H}))^{\perp}$ be the smooth unit normal vector field of $\varphi(\mathcal{H})$ and $\mathcal{R} \in \Gamma\left(S^{4} T^{*} \varphi(\mathcal{H})\right)$ be the Riemannian curvature of the surface $\varphi(\mathcal{H})$. Since the ambient space $\mathcal{S}=\mathbb{R}^{3}$ is flat, the Gauss and Codazzi-Mainardi equations for the Riemannian manifold $(\varphi(\mathcal{H}), \boldsymbol{g})$ read

$$
\begin{aligned}
\mathcal{R}(\boldsymbol{x}, \boldsymbol{y}, \boldsymbol{z}, \boldsymbol{w}) & =\boldsymbol{\beta}(\boldsymbol{x}, \boldsymbol{z}) \boldsymbol{\beta}(\boldsymbol{y}, \boldsymbol{w})-\boldsymbol{\beta}(\boldsymbol{x}, \boldsymbol{w}) \boldsymbol{\beta}(\boldsymbol{y}, \boldsymbol{z}) \\
\left(\nabla_{\boldsymbol{x}} \boldsymbol{\beta}\right)(\boldsymbol{y}, \boldsymbol{z}) & =\left(\nabla_{\boldsymbol{y}} \boldsymbol{\beta}\right)(\boldsymbol{z}, \boldsymbol{z}) .
\end{aligned}
$$




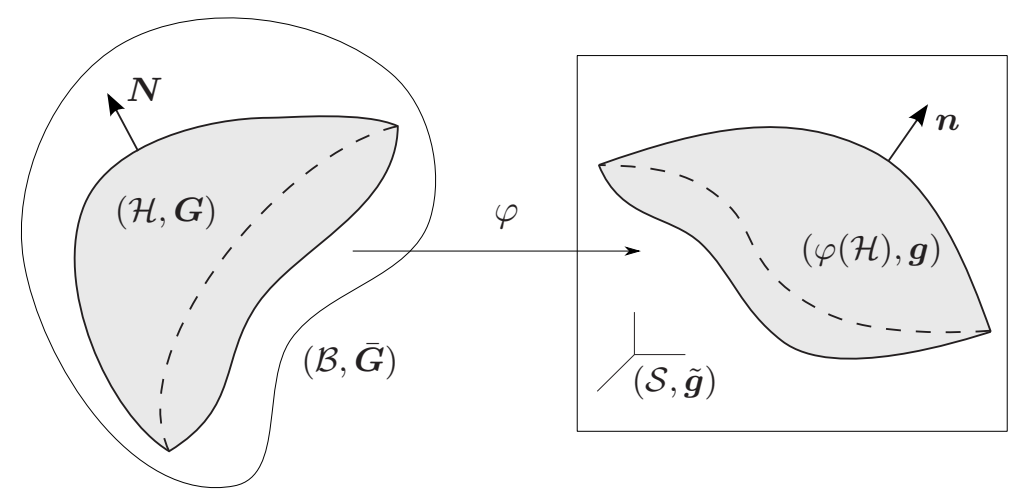

Figure 2: A configuration $\varphi: \mathcal{H} \rightarrow \mathcal{S}$ of a Riemannian surface $(\mathcal{H}, \boldsymbol{G})$ in the ambient space $(\mathcal{S}, \tilde{\boldsymbol{g}})$. The vector fields $\boldsymbol{N}$ and $\boldsymbol{n}$ are the unit normal vector fields of $\mathcal{H}$ and $\varphi(\mathcal{H})$, respectively.

We denote objects and indices by uppercase characters in the material manifold (e.g., $X \in \mathcal{H}$ for a material point) and by lowercase characters in the spatial manifold (e.g., $x \in \varphi(\mathcal{H})$ for a spatial point). In the remainder of the paper, unless stated otherwise, all indices (material and spatial) take values in the range 1,2. We adopt the standard Einstein convention of summation over repeated indices.

\subsection{Strain measures}

We define the deformation gradient $\boldsymbol{F}$ as the tangent map of $\varphi: \mathcal{H} \rightarrow \varphi(\mathcal{H})$, i.e., $\boldsymbol{F}(X):=T_{X} \varphi: T_{X} \mathcal{H} \mapsto$ $T_{\varphi(X)} \varphi(\mathcal{H})$. The right Cauchy-Green deformation tensor $\boldsymbol{C} \in \Gamma\left(S^{2} T^{*} \mathcal{H}\right)$ is defined as the pull back of the spatial metric [Marsden and Hughes, 1983], $\boldsymbol{C}(X):=\varphi^{*} \boldsymbol{g}(X): T_{X} \mathcal{H} \mapsto T_{X} \mathcal{H}$, i.e., $\boldsymbol{C}(\boldsymbol{X}, \boldsymbol{Y})=\boldsymbol{g}\left(\varphi_{*} \boldsymbol{X}, \varphi_{*} \boldsymbol{Y}\right)$, $\forall \boldsymbol{X}, \boldsymbol{Y} \in \mathfrak{X}(\mathcal{H})$. In components, $C_{A B}=F^{a}{ }_{A} F^{b}{ }_{B} g_{a b}$. The Jacobian of the motion $J$ relates the material and spatial Riemannian surface forms $d S(X, \boldsymbol{G})$ and $d s(\varphi(X), \boldsymbol{g})$ by

$$
\varphi^{*} d s=J d S .
$$

It can be shown that [Marsden and Hughes, 1983]

$$
J=\sqrt{\frac{\operatorname{det} \varphi^{*} \boldsymbol{g}}{\operatorname{det} \boldsymbol{G}}} .
$$

The material strain tensor $\boldsymbol{E} \in \Gamma\left(S^{2} T^{*} \mathcal{H}\right)$ is given by $\boldsymbol{E}=\frac{1}{2}(\boldsymbol{C}-\boldsymbol{G})$. The spatial strain tensor $\boldsymbol{e} \in$ $\Gamma\left(S^{2} T^{*} \varphi(\mathcal{H})\right)$ is defined as $\boldsymbol{e}=\frac{1}{2}(\boldsymbol{g}-\boldsymbol{c})$, where $\boldsymbol{c}=\varphi_{*} \boldsymbol{G}$. Note that $\boldsymbol{e}=\varphi_{*} \boldsymbol{E}$. The material and spatial strain tensors are intrinsic in the sense that they are determined by the metrics of the reference and the final configurations of the surface. We introduce extrinsic strain tensors for configurations of surfaces that depend on the second fundamental form as follows. The extrinsic deformation tensor $\Theta \in \Gamma\left(S^{2} T^{*} \mathcal{H}\right)$ is defined as the pull back of the spatial second fundamental form

$$
\Theta:=\varphi^{*} \boldsymbol{\beta} .
$$

In components, $\Theta_{A B}=F^{a}{ }_{A} F^{b}{ }_{B} \beta_{a b}$. We define the extrinsic material strain tensor as $\boldsymbol{H}:=\frac{1}{2}(\boldsymbol{\Theta}-\boldsymbol{B})$, and the extrinsic spatial strain tensor as $\boldsymbol{\eta}:=\frac{1}{2}(\boldsymbol{\beta}-\boldsymbol{\theta})$, where $\boldsymbol{\theta}:=\varphi_{*} \boldsymbol{B}$. Note that $\boldsymbol{\eta}=\varphi_{*} \boldsymbol{H}$. As an example, consider two different configurations of a sheet shown in Figure 3. The configuration $\varphi_{1}$ is an isometry between the sheet and a section of a cylinder, and therefore, $\boldsymbol{E}=\mathbf{0}$. However, note that since the out-of-plane geometry has changed, we have $\boldsymbol{H} \neq \mathbf{0}$. On the other hand, $\varphi_{2}$ is an in-plane deformation of the sheet with $\boldsymbol{E} \neq \mathbf{0}$, and $\boldsymbol{H}=\mathbf{0}$. 


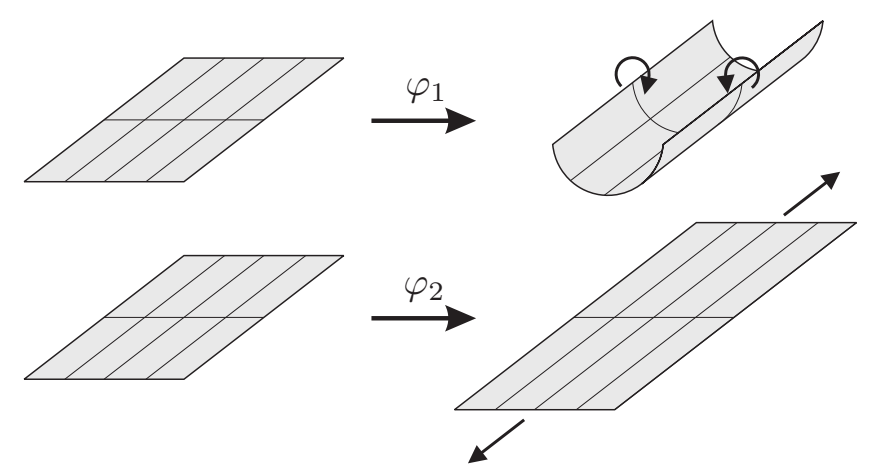

Figure 3: Strains of two different configurations of a sheet: (i) The configuration $\varphi_{1}$ maps the sheet to a section of a cylinder with $\boldsymbol{E}=\mathbf{0}$, and $\boldsymbol{H} \neq \mathbf{0}$, (ii) the configuration $\varphi_{2}$ is an in-plane extension of the sheet with $\boldsymbol{E} \neq \mathbf{0}$, and $\boldsymbol{H}=\mathbf{0}$.

\subsection{Compatibility equations of shells}

The pull-back of the Gauss and the Codazzi equations $(3.1)$ of the surface $(\varphi(\mathcal{H}), \boldsymbol{g})$ by $\varphi$ read $[$ Angoshtari and Yavari, 2015]

$$
\begin{aligned}
\mathcal{R}^{C}(\boldsymbol{X}, \boldsymbol{Y}, \boldsymbol{Z}, \boldsymbol{W}) & =\boldsymbol{\Theta}(\boldsymbol{X}, \boldsymbol{Z}) \boldsymbol{\Theta}(\boldsymbol{Y}, \boldsymbol{W})-\boldsymbol{\Theta}(\boldsymbol{X}, \boldsymbol{W}) \boldsymbol{\Theta}(\boldsymbol{Y}, \boldsymbol{Z}), \\
\left(\nabla_{\boldsymbol{X}}^{C} \boldsymbol{\Theta}\right)(\boldsymbol{Y}, \boldsymbol{Z}) & =\left(\nabla_{\boldsymbol{Y}}^{\boldsymbol{C}} \boldsymbol{\Theta}\right)(\boldsymbol{X}, \boldsymbol{Z}),
\end{aligned}
$$

where $\nabla^{C}$ and $\mathcal{R}^{C}$ are, respectively, the Levi-Civita connection and the Riemannian curvature of the Riemannian manifold $(\mathcal{H}, \boldsymbol{C})$. Given a metric $\boldsymbol{C} \in \Gamma\left(S^{2} T^{*} \mathcal{H}\right)$ and a symmetric tensor $\boldsymbol{\Theta} \in \Gamma\left(S^{2} T^{*} \mathcal{H}\right)$, the relations (3.3) express the compatibility equations for these tensors when $\mathcal{H}$ is simply-connected, i.e., they are the necessary and locally sufficient conditions for the existence of a configuration of $\mathcal{H}$ with the given deformation tensors that is unique up to isometries of $\mathcal{S}=\mathbb{R}^{3}$ when $\mathcal{H}$ is simply-connected [Ivey and Landsberg, 2003; Angoshtari and Yavari, 2015]. Hence, we observe that if $\varphi_{1}$ and $\varphi_{2}$ are different configurations of the surface $\mathcal{H} \subset \mathbb{R}^{3}$ with the same deformation tensors, then $\varphi_{1} \circ \varphi_{2}^{-1}$ and $\varphi_{2} \circ \varphi_{1}^{-1}$ are rigid body motions of $\mathcal{H}$ in $\mathbb{R}^{3}$. Note that similar to (2.4), given the symmetries of the curvature tensor $\mathcal{R}^{C}$ and the extrinsic deformation tensor, the compatibility equations reduce in components to

$$
\begin{aligned}
& \mathcal{R}_{1212}^{C}=\Theta_{11} \Theta_{22}-\Theta_{12} \Theta_{12}, \\
& \Theta_{11 \| 2}=\Theta_{12 \| 1}, \\
& \Theta_{22 \| 1}=\Theta_{12 \| 2},
\end{aligned}
$$

where we denote by a double stroke $\|$ the covariant derivative corresponding to the Levi-Civita connection of $(\mathcal{H}, \boldsymbol{C})$.

\subsection{Stress-free shell growth}

Given a thin body $\mathcal{B}$ and its idealization, the mid-surface $\mathcal{H}$, we want to find those growth fields that leave the shell "stress-free", by which we mean both stress and couple-stress free. As introduced earlier, given the smooth embedding $\varphi$ of the surface $(\mathcal{H}, \boldsymbol{G}, \boldsymbol{B})$ into the Euclidean space $\mathcal{S}$ to form a surface $(\varphi(\mathcal{H}), \boldsymbol{g}, \boldsymbol{\beta})$, the tensors $2 \boldsymbol{E}=\varphi^{*} \boldsymbol{g}-\boldsymbol{G}$ and $2 \boldsymbol{H}=\varphi^{*} \boldsymbol{\beta}-\boldsymbol{B}$, respectively, provide measures of in-plane and out-of-plane strains. Therefore, the surface is stress-free when these two measures are identically zero, i.e., $\varphi^{*} \boldsymbol{g}=\boldsymbol{G}$ and $\varphi^{*} \boldsymbol{\beta}=\boldsymbol{B}$. Noting that $\boldsymbol{C}=\varphi^{*} \boldsymbol{g}$ and $\boldsymbol{\Theta}:=\varphi^{*} \boldsymbol{\beta}$ are uniquely specified by (3.3) when $\mathcal{H}$ is simply-connected, it follows that a simply-connected shell $\mathcal{H}$ is stress-free if and only if $\boldsymbol{G}$ and $\boldsymbol{B}$ are specified by (3.3), i.e., we have

$$
\begin{aligned}
\mathcal{R}^{\mathcal{H}}(\boldsymbol{X}, \boldsymbol{Y}, \boldsymbol{Z}, \boldsymbol{W}) & =\boldsymbol{B}(\boldsymbol{X}, \boldsymbol{W}) \boldsymbol{B}(\boldsymbol{Y}, \boldsymbol{Z})-\boldsymbol{B}(\boldsymbol{X}, \boldsymbol{Z}) \boldsymbol{B}(\boldsymbol{Y}, \boldsymbol{W}) \\
\left(\nabla_{\boldsymbol{Y}}^{\mathcal{H}} \boldsymbol{B}\right)(\boldsymbol{X}, \boldsymbol{Z}) & =\left(\nabla_{\boldsymbol{X}}^{\mathcal{H}} \boldsymbol{B}\right)(\boldsymbol{Y}, \boldsymbol{Z}) .
\end{aligned}
$$




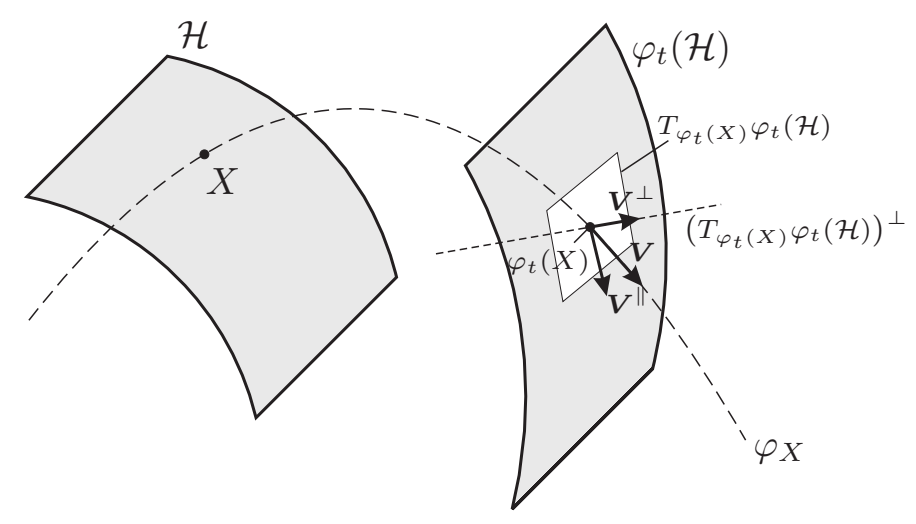

Figure 4: The decomposition of the material velocity $\boldsymbol{V}=\boldsymbol{V}^{\|}+\boldsymbol{V}^{\perp}$. The component $\boldsymbol{V}^{\|}(X, t)$ is an element of $T_{\varphi(X)} \varphi(\mathcal{H})$ and $\boldsymbol{V}^{\perp}(X, t)$ is normal to $T_{\varphi(X)} \varphi(\mathcal{H})$.

These are precisely the necessary and sufficient conditions for the simply-connected surface $(\mathcal{H}, \boldsymbol{G}, \boldsymbol{B})$ to be isometrically embeddable in $\mathbb{R}^{3}$. In components, equations (3.5) reduce to

$$
\begin{aligned}
\mathcal{R}_{1221}^{\mathcal{H}} & =B_{11} B_{22}-B_{12} B_{12}, \\
B_{11 \mid 2} & =B_{12 \mid 1}, \\
B_{22 \mid 1} & =B_{12 \mid 2} .
\end{aligned}
$$

\subsection{Velocity and acceleration}

We define a motion to be a smooth curve $t \mapsto \varphi_{t} \in \mathcal{C}$, i.e., $\varphi_{t}: \mathcal{H} \rightarrow \mathcal{S}$ and denote $\varphi(., t):=\varphi_{t}($.$) and$ $\varphi_{X}():.=\varphi(X,$.$) . At time t$, the surface $\varphi_{t}(\mathcal{H})$ has the metric $\boldsymbol{g}:=\left.\tilde{\boldsymbol{g}}\right|_{\varphi_{t}(\mathcal{H})}$, the Levi-Civita connection $\nabla$, the unit normal vector field $\boldsymbol{n} \in \mathfrak{X}\left(\varphi_{t}(\mathcal{H})\right)^{\perp}$, and the second fundamental form $\boldsymbol{\beta} \in \Gamma\left(S^{2} T^{*} \varphi_{t}(\mathcal{H})\right)$. The material velocity is the mapping $\boldsymbol{V}: \mathcal{H} \times \mathbb{R} \rightarrow T \mathcal{S},(X, t) \mapsto \boldsymbol{V}(X, t):=T_{t} \varphi_{X}\left[\partial_{t}\right], \forall X \in \mathcal{H}$. We denote for each $X \in \mathcal{H}, \boldsymbol{V}_{X}:=\boldsymbol{V}(X, \cdot)$ the vector field along the curve $\varphi_{X}$, i.e., $\boldsymbol{V}_{X} \in \mathfrak{X}\left(\varphi_{X}\right)$. Using the decomposition of $T \mathcal{S}$, the material velocity can be decomposed as $\boldsymbol{V}_{X}(t)=\boldsymbol{V}_{X}^{\|}(t)+\boldsymbol{V}_{X}^{\perp}(t)$, where $\boldsymbol{V}_{X}^{\|}(t) \in T_{\varphi_{t}}(X) \varphi_{t}(\mathcal{H})$ and $\boldsymbol{V}_{X}^{\perp}(t) \in\left(T_{\varphi_{t}(X)} \varphi_{t}(\mathcal{H})\right)^{\perp}$, i.e., $\boldsymbol{V}^{\|}$is parallel to $\varphi_{t}(\mathcal{H})$ and $\boldsymbol{V}^{\perp}$ is normal to $\varphi_{t}(\mathcal{H})$, see Figure 4 . The spatial velocity at a fixed time $t$ is a vector field along $\varphi_{t}(\mathcal{H})$ defined as $\boldsymbol{v}(x, t):=\boldsymbol{V}\left(\varphi_{t}^{-1}(X), t\right)$, where $x=\varphi_{t}^{-1}(X) \in \varphi_{t}(\mathcal{H}) \subset \mathcal{S}$. Note that even though for a fixed $t$, the mapping $\varphi_{t}: \mathcal{H} \rightarrow \mathcal{S}$ is a smooth embedding, the mapping $\varphi: \mathcal{H} \times \mathbb{R} \rightarrow \mathcal{S}$ is, in general, not even an immersion. In fact, it can be seen that $T_{(X, t)} \varphi$ is not necessarily injective. In $\left\{X^{A}\right\}$ and $\left\{x^{a}\right\}$, some local coordinate charts for $\mathcal{H}$ and $\mathcal{S}$, respectively, $T_{(X, t)} \varphi$ reads as follows

$$
T_{(X, t)} \varphi=\left(\begin{array}{lll}
\frac{\partial \varphi^{1}}{\partial X^{1}} & \frac{\partial \varphi^{1}}{\partial X^{2}} & \frac{\partial \varphi^{1}}{\partial t^{2}} \\
\frac{\partial \varphi^{2}}{\partial X_{1}^{1}} & \frac{\partial \varphi^{2}}{\partial X^{2}} & \frac{\partial \varphi^{2}}{\partial t_{3}} \\
\frac{\partial \varphi^{3}}{\partial X^{1}} & \frac{\partial \varphi^{3}}{\partial X^{2}} & \frac{\partial \varphi^{3}}{\partial t}
\end{array}\right)
$$

Now, if $\boldsymbol{V}(X, t)=\mathbf{0}$ (i.e., $\partial \varphi^{a} / \partial t=0$ for $a=1,2,3$ ), or $\varphi$ is an in-plane motion (i.e., in some coordinate chart for $\mathcal{S}$ such that $\partial_{3}=\boldsymbol{n}$ on $\varphi_{t}(\mathcal{H})$ we have $\left.\varphi^{3}=0\right), T_{(X, t)} \varphi$ is clearly not injective. However, if $T_{(X, t)} \varphi$ is injective, the implicit function theorem implies that $\varphi$ is a local diffeomorphism at $(X, t)$, and one can construct a local vector field $\mathcal{V}$ on $\mathcal{S}$ in a neighborhood of $\varphi(X, t)$ such that $\mathcal{V}(\varphi(X, t))=\boldsymbol{V}(X, t)=\boldsymbol{v}(\varphi(X, t), t)$. Hence, the material acceleration can be in this case unambiguously defined as

$$
\boldsymbol{A}(X, t)=D_{\varphi_{X}} \boldsymbol{V}_{X}:=\tilde{\nabla} \mathcal{v} \mathcal{V}(\varphi(X, t))
$$

where $D_{\varphi_{X}}$ is the covariant derivative along $\varphi_{X}$. Using the decomposition of the material velocity into parallel and normal components $\mathcal{V}=\mathcal{V}^{\|}+\mathcal{V}^{\perp}$ and assuming that $\varphi$ is a local diffeomorphism at $(X, t)$, one can write

$$
\boldsymbol{A}(X, t)=\tilde{\nabla} v\left(\mathcal{V}^{\|}+\mathcal{V}^{\perp}\right)=\tilde{\nabla} v \mathcal{V}^{\|}+\tilde{\nabla} v \mathcal{V}^{\perp}
$$


Since $\nabla$ is the Levi-Civita connection on $T \varphi(\mathcal{H})$ induced by $\boldsymbol{g}$, it is torsion free and hence

$$
\tilde{\nabla}_{\mathcal{v}} \mathcal{V}^{\|}=\left[\mathcal{V}, \mathcal{v}^{\|}\right]+\tilde{\nabla}_{\mathcal{v} \|} \mathcal{V}=\left[\mathcal{V}, \mathcal{v}^{\|}\right]+\tilde{\nabla}_{\mathcal{v} \|} \mathcal{V}^{\|}+\tilde{\nabla}_{\mathcal{v} \|} \mathcal{v}^{\perp}
$$

Note that since $\mathcal{V}=\mathcal{V}(\tilde{\varphi}(X, t))$ does not explicitly depend on time, hence, denoting the Lie derivative by $\boldsymbol{L}$, one can write $\left[\mathcal{V}, \mathcal{V}_{\|}\right]=\boldsymbol{L}_{\mathcal{V}} \mathcal{V}_{\|}$, which is tangent to $\mathcal{S}_{t} .{ }^{3}$ Following the definition of the second fundamental form, we have $\tilde{\nabla}_{\mathcal{V} \|} \mathcal{V}^{\|}=\nabla_{\mathcal{V} \|} \mathcal{V}^{\|}+\boldsymbol{\beta}\left(\mathcal{V}^{\|}, \mathcal{V}^{\|}\right) \boldsymbol{n}$. We let $\mathcal{V}^{n}=\tilde{\boldsymbol{g}}(\mathcal{V}, \boldsymbol{n})$, i.e., $\mathcal{V}^{\perp}=\mathcal{V}^{n} \boldsymbol{n}$. The metric compatibility of $\tilde{\nabla}$ and the fact that $\tilde{\boldsymbol{g}}(\boldsymbol{n}, \boldsymbol{n})=1$ imply that for any vector $\boldsymbol{W}$ along $\varphi_{t}(\mathcal{H})$ in $\mathcal{S}$, we have

$$
\frac{d}{d t}(\tilde{\boldsymbol{g}}(\boldsymbol{n}, \boldsymbol{n}))=2 \tilde{\boldsymbol{g}}\left(\tilde{\nabla}_{\boldsymbol{W}} \boldsymbol{n}, \boldsymbol{n}\right)=0,
$$

i.e., $\tilde{\nabla}_{\boldsymbol{W}} \boldsymbol{n} \in \mathfrak{X}(\varphi(\mathcal{H}))$. Thus $\tilde{\nabla}_{\mathcal{v} \|} \boldsymbol{n}=-\boldsymbol{g}^{\sharp} \cdot \boldsymbol{\beta} \cdot \boldsymbol{v}^{\|}$. Therefore

$$
\tilde{\nabla}_{\mathcal{V} \|} \mathcal{V}^{\perp}=\tilde{\nabla}_{\mathcal{V} \|}\left(\mathcal{V}^{n} \boldsymbol{n}\right)=\tilde{\nabla}_{\mathcal{V} \|}\left(\mathcal{V}^{n}\right) \boldsymbol{n}+\mathcal{V}^{n} \tilde{\nabla}_{\mathcal{V} \|} \boldsymbol{n}=\left(d \mathcal{V}^{n} \cdot \mathcal{V}^{\|}\right) \boldsymbol{n}-\mathcal{V}^{n} \boldsymbol{g}^{\sharp} \cdot \boldsymbol{\beta} \cdot \mathcal{V}^{\|}
$$

On the other hand, we have

$$
\tilde{\nabla} \mathcal{V} \mathcal{V}^{\perp}=\tilde{\nabla}_{\mathcal{V}}\left(\mathcal{V}^{n} \boldsymbol{n}\right)=\frac{d \mathcal{V}^{n}}{d t} \boldsymbol{n}+\mathcal{V}^{n} \tilde{\nabla}_{\mathcal{V}} \boldsymbol{n}
$$

However, as observed earlier, $\tilde{\nabla} v \boldsymbol{n} \in \mathfrak{X}(\varphi(\mathcal{H}))$, then, it follows that at $\varphi(X, t)$, one can write

$$
\left(\tilde{\nabla} \mathcal{V} \mathcal{V}^{\perp}\right)^{\perp}=\frac{d V^{n}}{d t} \boldsymbol{n}
$$

Let us now compute $\left(\tilde{\nabla} \mathcal{V} \mathcal{V}^{\perp}\right)^{\|}$. We consider an arbitrary vector field $\boldsymbol{U}$ in $\mathcal{S}$ such that $\boldsymbol{U}$ is tangent to $\mathcal{H}$ in a neighborhood of $\varphi(X, t)$, i.e., $\tilde{\boldsymbol{g}}\left(\mathcal{V}^{\perp}, \boldsymbol{U}\right)=0$. Hence, $\tilde{\boldsymbol{g}}\left(\tilde{\nabla} \mathcal{V} \mathcal{V}^{\perp}, \boldsymbol{U}\right)=-\tilde{\boldsymbol{g}}\left(\mathcal{V}^{\perp}, \tilde{\nabla} \mathcal{V} \boldsymbol{U}\right)$. However, at $\varphi(X, t)$, we have

$$
\begin{aligned}
\tilde{\nabla}_{\mathcal{V}} \boldsymbol{U} & =[\mathcal{V}, \boldsymbol{U}]+\tilde{\nabla}_{\boldsymbol{U}} \mathcal{V}=[\mathcal{V}, \boldsymbol{U}]+\tilde{\nabla}_{\boldsymbol{U}} \mathcal{V}^{\|}+\tilde{\nabla}_{\boldsymbol{U}} \mathcal{V}^{\perp} \\
& =[\mathcal{V}, \boldsymbol{U}]+\nabla_{\boldsymbol{U}} \boldsymbol{V}^{\|}+\boldsymbol{\beta}\left(\boldsymbol{V}^{\|}, \boldsymbol{U}\right) \boldsymbol{n}+\left(d V^{n} \cdot \boldsymbol{U}\right) \boldsymbol{n}+V^{n} \tilde{\nabla}_{\boldsymbol{U}} \boldsymbol{n}
\end{aligned}
$$

Hence $^{4}$

$$
\tilde{\boldsymbol{g}}\left(\mathcal{V}^{\perp}, \tilde{\nabla} \mathcal{V} \boldsymbol{U}\right)=V^{n} \boldsymbol{\beta}\left(\boldsymbol{V}^{\|}, \boldsymbol{U}\right)+V^{n}\left(d V^{n} \cdot \boldsymbol{U}\right)
$$

Thus, it follows from $\tilde{\boldsymbol{g}}\left(\tilde{\nabla} \mathcal{V} \mathcal{V}^{\perp}, \boldsymbol{U}\right)=-\tilde{\boldsymbol{g}}\left(\mathcal{V}^{\perp}, \tilde{\nabla} \mathcal{V} \boldsymbol{U}\right)$ and by arbitrariness of $\boldsymbol{U}$ that

$$
\left(\tilde{\nabla} \mathcal{V} \mathcal{V}^{\perp}\right)^{\|}=-\mathcal{V}^{n} \boldsymbol{g}^{\sharp} \cdot \boldsymbol{\beta} \cdot \mathcal{V}^{\|}-\mathcal{V}^{n}\left(d \mathcal{V}^{n}\right)^{\sharp} .
$$

Therefore, the parallel and normal components of the material acceleration read

$$
\begin{aligned}
\boldsymbol{A}^{\|} & =\left[\boldsymbol{V}, \boldsymbol{V}^{\|}\right]+\nabla_{\boldsymbol{V}^{\|}} \boldsymbol{V}^{\|}-2 V^{n} \boldsymbol{g}^{\sharp} \cdot \boldsymbol{\beta} \cdot \boldsymbol{V}^{\|}-V^{n}\left(d V^{n}\right)^{\sharp}, \\
\boldsymbol{A}^{\perp} & =\left[\frac{d V^{n}}{d t}+\boldsymbol{\beta}\left(\boldsymbol{V}^{\|}, \boldsymbol{V}^{\|}\right)+d V^{n} \cdot \boldsymbol{V}^{\|}\right] \boldsymbol{n} .
\end{aligned}
$$

\section{The governing equations of motion}

In this section we derive the governing equations of motion for morphoelastic shells that include: balance of mass, balance of linear and angular momenta, and the kinetic equations of growth.

\footnotetext{
${ }^{3}$ The Lie derivative along the vector field $\mathcal{V}$ is defined as $\boldsymbol{L}_{\mathcal{V}} \mathcal{V}_{\|}=\left.\frac{d}{d t}\right|_{t=s}\left[\left(\varphi_{t} \circ \varphi_{s}^{-1}\right)^{*} \mathcal{V}_{\|}\right]$, where $\varphi_{t} \circ \varphi_{s}^{-1}$ is the flow of $\mathcal{V}$

${ }^{4}$ Note that since the vector $\boldsymbol{U}$ is tangent to $\mathcal{H}$ at $\varphi(X, t)$, the vectors $[\mathcal{V}, \boldsymbol{U}]=\boldsymbol{L}_{\mathcal{V}} \boldsymbol{U}$ and $\tilde{\nabla}_{\boldsymbol{U}} \boldsymbol{n}$ are tangent to $\mathcal{H}$ as well.
} 


\subsection{Balance of mass}

We denote the material and spatial surface mass densities (mass per unit area) by $\rho$ and $\varrho$, respectively, and let $\mathcal{U}$ be any open set in $\mathcal{H}$ with a smooth boundary. For a growing body, the balance of mass for a motion $\varphi$ can be written as

$$
\frac{d}{d t} \int_{\mathcal{U}} \rho d S=\int_{\mathcal{U}} S_{m} d S
$$

where $S_{m}=S_{m}(X, t)$ is a given scalar field characterizing the material rate of change of mass per unit area. We postulate that the motion $\varphi$ conserves the mass of the system, i.e.

$$
\int_{\varphi_{t}(\mathcal{U})} \varrho d s=\int_{\mathcal{U}} \rho d S .
$$

Recalling that $d S=\sqrt{\operatorname{det} \boldsymbol{G}} d X^{1} \wedge d X^{2}$, it follows from (4.1) that $\rho=\rho(X, \boldsymbol{G}, t)$, and we find

$$
\frac{1}{\sqrt{\operatorname{det} \boldsymbol{G}}} \frac{d}{d t}(\sqrt{\operatorname{det} \boldsymbol{G} \rho})=S_{m},
$$

which, using the identity $\frac{d}{d t}[\operatorname{det} \boldsymbol{A}(t)]=\operatorname{det} \boldsymbol{A}(t) \operatorname{tr}\left[\boldsymbol{A}^{-1}(t) \frac{d}{d t} \boldsymbol{A}(t)\right]$, gives the material local form of the balance of mass for a growing body as

$$
\dot{\rho}+\frac{1}{2} \rho \operatorname{tr} \dot{\boldsymbol{G}}=S_{m},
$$

where the dot denotes total time differentiation. Note that if we write the evolving metric as in (2.10), i.e., $\boldsymbol{G}(X, t)=\boldsymbol{G}^{0}(X) e^{2 \boldsymbol{\omega}(X, t)}$, and use the identity $\operatorname{det}\left(e^{\boldsymbol{A}}\right)=e^{\operatorname{tr}(\boldsymbol{A})}$, then (4.3) reads

$$
\dot{\rho}+\rho \frac{d}{d t}[\operatorname{tr}(\boldsymbol{\omega})]=S_{m} .
$$

Now, since $J=\sqrt{\frac{\operatorname{det} \varphi^{*} \boldsymbol{g}}{\operatorname{det} \boldsymbol{G}}}=J(X, \varphi, \boldsymbol{G}, \boldsymbol{g})$, and $\rho=J \varrho$, it follows from (4.4) that $\varrho=\varrho(X, \varphi, \boldsymbol{G}, \boldsymbol{g}, t)$, and we find $^{5}$

$$
\dot{\varrho}+\varrho \frac{\dot{J}}{J}+\frac{1}{2} \varrho \operatorname{tr}\left(\frac{d \boldsymbol{G}}{d t}\right)=s_{m},
$$

where $s_{m}(X, t)=\frac{1}{J} S_{m}(X, t)$ is the spatial rate of change of mass per unit area. Using (3.2), one can write

$$
\dot{J}=\frac{1}{2} \frac{d}{d t}\left(\operatorname{det} \varphi^{*} \boldsymbol{g}\right) \frac{1}{\sqrt{\operatorname{det} \boldsymbol{G} \operatorname{det} \varphi^{*} \boldsymbol{g}}}-\frac{1}{2} \frac{d}{d t}(\operatorname{det} \boldsymbol{G}) \sqrt{\frac{\operatorname{det} \varphi^{*} \boldsymbol{g}}{(\operatorname{det} \boldsymbol{G})^{3}}} .
$$

Therefore

$$
\frac{\dot{J}}{J}=\frac{1}{2} \operatorname{tr}_{\boldsymbol{C}}\left(\frac{d \varphi^{*} \boldsymbol{g}}{d t}\right)-\frac{1}{2} \operatorname{tr}\left(\frac{d \boldsymbol{G}}{d t}\right),
$$

where $\operatorname{tr}_{\boldsymbol{C}}$ is the trace taken with respect to the metric $\boldsymbol{C}$. Recalling the decomposition $\boldsymbol{v}=\boldsymbol{v}^{\|}+v^{n} \boldsymbol{n}$, we have [Marsden and Hughes, 1983; Verpoort, 2008; Kadianakis and Travlopanos, 2013]

$$
\varphi_{*} \frac{d \varphi^{*} \boldsymbol{g}}{d t}=\boldsymbol{L}_{\boldsymbol{v}} \boldsymbol{g}=\boldsymbol{L}_{\boldsymbol{v} \|} \boldsymbol{g}-2 v^{n} \boldsymbol{\beta}
$$

and it follows that

$$
\operatorname{tr}_{\boldsymbol{C}}\left(\frac{d \varphi^{*} \boldsymbol{g}}{d t}\right)=2 \operatorname{div} \boldsymbol{v}^{\|}-2 v^{n} \operatorname{tr} \boldsymbol{\beta}
$$

where div denotes the divergence on the surface $\varphi_{t}(\mathcal{H})$. Therefore, $(4.5)$ gives the spatial local form of the balance of mass for a growing shell as $\dot{\varrho}+\varrho \operatorname{div} \boldsymbol{v}^{\|}-\varrho v^{n} \operatorname{tr} \boldsymbol{\beta}=s_{m}$.

${ }^{5}$ Note that (4.5) can also be obtained from (4.2) and (4.1) by writing

$$
\frac{d}{d t} \int_{\varphi_{t}(\mathcal{U})} \varrho d s=\int_{\mathcal{U}} S_{m} d S .
$$




\subsection{Balance laws}

Given the right Cauchy-Green deformation tensor $\boldsymbol{C}$ and the extrinsic deformation tensor $\boldsymbol{\Theta}$, the geometry of the deformed surface is uniquely defined (See $\S 3.2$ ). However, in order to specify the evolution of an element of the deformed surface, we need to know its position $\varphi$ and its orientation by means of the normal vector field $\mathcal{N}$. Therefore, in the classical theory of nonlinear elasticity of shells, we define the action functional as the map $S: \mathcal{C} \mapsto \mathbb{R}$

$$
S(\varphi)=\int_{t_{0}}^{t_{1}} \int_{\mathcal{H}} \mathcal{L}(X, \varphi(X, t), \mathcal{N} \circ \varphi(X, t), \dot{\varphi}(X, t), \tilde{\boldsymbol{g}} \circ \varphi(X, t), \boldsymbol{C}(X, t), \boldsymbol{\Theta}(X, t), \boldsymbol{G}(X)) d S(X) d t,
$$

where $\mathcal{L}=\mathcal{L}(X, \varphi, \mathcal{N}, \dot{\varphi}, \tilde{\boldsymbol{g}}, \boldsymbol{C}, \boldsymbol{\Theta}, \boldsymbol{G})$ is the Lagrangian density per unit surface area. ${ }^{6}$ The governing equations of motion follow from Hamilton's principle of least action, which states that the physical motion $\varphi$ of $\mathcal{H}$ between $t_{0}$ and $t_{1}$ is a critical point for the action functional, i.e.

$$
\delta S(\varphi)=0 .
$$

In the present geometric theory of morphoelastic shells, the material first and second fundamental forms are dynamical variables that vary independently of the motion. Therefore, the action functional is modified to read

$S(\varphi, \boldsymbol{G}, \boldsymbol{B})=\int_{t_{0}}^{t_{1}} \int_{\mathcal{H}} \mathcal{L}(X, \varphi(X, t), \mathcal{N} \circ \varphi(X, t), \dot{\varphi}(X, t), \tilde{\boldsymbol{g}} \circ \varphi(X, t), \boldsymbol{C}(X, t), \boldsymbol{\Theta}(X, t), \boldsymbol{G}(X, t), \boldsymbol{B}(X, t)) d S(X, t) d t$.

We recall that $d S(X, t)=\sqrt{\operatorname{det} \boldsymbol{G}(X, t)} d X^{1} \wedge d X^{2}$, and define the Lagrangian density $\mathcal{L}$ by

$$
\mathcal{L}(X, \varphi, \mathcal{N}, \dot{\varphi}, \tilde{\boldsymbol{g}}, \boldsymbol{C}, \boldsymbol{\Theta}, \boldsymbol{G}, \boldsymbol{B})=\frac{1}{2} \rho \tilde{\boldsymbol{g}}(\dot{\varphi}, \dot{\varphi})-\mathcal{W}(X, \boldsymbol{C}, \boldsymbol{\Theta}, \boldsymbol{G}, \boldsymbol{B})-\mathcal{V}(X, \varphi, \mathcal{N}, \tilde{\boldsymbol{g}}),
$$

where $\mathcal{W}=\mathcal{W}(X, \boldsymbol{C}, \boldsymbol{\Theta}, \boldsymbol{G}, \boldsymbol{B})$ is the elastic energy density per unit surface area (related to the elastic deformation of a surface element), and $\mathcal{V}=\mathcal{V}(X, \varphi, \mathcal{N}, \tilde{\boldsymbol{g}})$ is the potential energy density per unit surface area (related to the position - respectively orientation - of a surface element in the body force - respectively moment-fields). Similar to the coordinate chart $\left(X^{1}, X^{2}, X^{3}\right)$ previously defined for $\mathcal{B}$, let $\left(x^{1}, x^{2}, x^{3}\right)$ be a local coordinate chart for $\mathcal{S}$ such that at any point of the hypersurface $\varphi(\mathcal{H}),\left\{x^{1}, x^{2}\right\}$ is a local coordinate chart for $\varphi(\mathcal{H})$ and the normal vector field $\boldsymbol{n}$ to $\varphi(\mathcal{H})$ is tangent to the coordinate curve $x^{3}$. Therefore, the Lagrangian density in this coordinate chart reads

$$
\mathcal{L}(X, \varphi, \mathcal{N}, \dot{\varphi}, \tilde{\boldsymbol{g}}, \boldsymbol{C}, \boldsymbol{\Theta}, \boldsymbol{G}, \boldsymbol{B})=\frac{1}{2} \rho g_{a b} \dot{\varphi}^{a} \dot{\varphi}^{b}+\frac{1}{2} \rho\left(\dot{\varphi}^{n}\right)^{2}-\mathcal{W}(X, \boldsymbol{C}, \boldsymbol{\Theta}, \boldsymbol{G}, \boldsymbol{B})-\mathcal{V}(X, \varphi, \mathcal{N}, \tilde{\boldsymbol{g}})
$$

Also, because growth is, in general, a non-conservative process, we use Lagrange-d'Alembert's principle, which given non-conservative forces $\boldsymbol{F}_{\boldsymbol{\varphi}}, \boldsymbol{F}_{\mathcal{N}}, \boldsymbol{F}_{\boldsymbol{G}}$, and $\boldsymbol{F}_{\boldsymbol{B}}$, states that [Marsden and Ratiu, 1994]

$$
\delta S(\varphi, \boldsymbol{G}, \boldsymbol{B})+\int_{t_{0}}^{t_{1}} \int_{\mathcal{H}}\left(\boldsymbol{F}_{\varphi} \cdot \delta \varphi+\boldsymbol{F}_{\mathcal{N}} \cdot \delta \mathcal{N}+\boldsymbol{F}_{\boldsymbol{G}}: \delta \boldsymbol{G}+\boldsymbol{F}_{\boldsymbol{B}}: \delta \boldsymbol{B}\right) d S d t=0 .
$$

The sources of these forces depend on the particular underlying biological, biochemical, or physical processes leading to growth. Here, we assume the existence of a Rayleigh potential $\mathcal{R}=\mathcal{R}(\dot{\varphi}, \dot{\mathcal{N}}, \tilde{\boldsymbol{g}}, \dot{\boldsymbol{G}}, \dot{\boldsymbol{B}}, \boldsymbol{G})$ such that

$$
\boldsymbol{F}_{\varphi}=-\frac{\partial \mathcal{R}}{\partial \dot{\varphi}}, \quad \boldsymbol{F}_{\mathcal{N}}=-\frac{\partial \mathcal{R}}{\partial \dot{\mathcal{N}}}, \quad \boldsymbol{F}_{\boldsymbol{G}}=-\frac{\partial \mathcal{R}}{\partial \dot{\boldsymbol{G}}}, \quad \text { and } \quad \boldsymbol{F}_{\boldsymbol{B}}=-\frac{\partial \mathcal{R}}{\partial \dot{\boldsymbol{B}}} .
$$

In the context of our theory, we disregard non-conservative forces due to the variations of position and orientation and assume that it is only due to growth, i.e., we assume that $\mathcal{R}=\mathcal{R}(\dot{\boldsymbol{G}}, \dot{\boldsymbol{B}}, \boldsymbol{G})$.

In order to take variations, we let $\varphi_{\epsilon}$ be a 1-parameter family of motions such that $\varphi_{0, t}=\varphi_{t} \cdot{ }^{7}$ For fixed $X$ and $t$, we consider the curve $\varphi_{t, X}: \epsilon \mapsto \varphi_{t, X}(\epsilon):=\varphi_{\epsilon, t}(X)$, and define the variation of motion as the spatial vector field given by

$$
\delta \varphi(X, t)=\left.T_{\epsilon} \varphi_{t, X}\left[\partial_{\epsilon}\right]\right|_{\epsilon=0} \in T_{\varphi_{\epsilon, t}(X)} \mathcal{S}
$$

\footnotetext{
${ }^{6}$ Since the Lagrangian density is a scalar, it depends on the metrics $\boldsymbol{G}$ and $\tilde{\boldsymbol{g}}$

${ }^{7}$ For fixed $X$ and $t$, we let $\varphi_{\epsilon, t}(X):=\varphi_{\epsilon}(X, t)$.
} 
Similarly, we let $\boldsymbol{G}_{\epsilon}$ be a 1-parameter family of material metrics such that $\boldsymbol{G}_{\epsilon=0}=\boldsymbol{G}$ and for fixed $X$ and $t$, we define the variation of the metric by the material tensor given by

$$
\delta \boldsymbol{G}(X, t)=\left.\frac{d \boldsymbol{G}_{\epsilon}}{d \epsilon}\right|_{\epsilon=0}(X, t) .
$$

Also, let $\boldsymbol{B}_{\epsilon}$ be a 1-parameter family of second fundamental forms such that $\boldsymbol{B}_{\epsilon=0}=\boldsymbol{B}$ and for fixed $X$ and $t$, we define the variation of the second fundamental form by the material tensor given by

$$
\delta \boldsymbol{B}(X, t)=\left.\frac{d \boldsymbol{B}_{\epsilon}}{d \epsilon}\right|_{\epsilon=0}(X, t) .
$$

It follows from Lagrange-d'Alembert's principle that

$$
\begin{array}{r}
\int_{t_{0}}^{t_{1}} \int_{\mathcal{H}}\left(\frac{\partial \mathcal{L}}{\partial \varphi} \cdot \delta \varphi+\frac{\partial \mathcal{L}}{\partial \mathcal{N}} \cdot \delta \mathcal{N}+\frac{\partial \mathcal{L}}{\partial \dot{\varphi}} \cdot \delta \dot{\varphi}+\frac{\partial \mathcal{L}}{\partial \tilde{\boldsymbol{g}}}: \delta \tilde{\boldsymbol{g}} \circ \varphi+\frac{\partial \mathcal{L}}{\partial \boldsymbol{C}}: \delta \boldsymbol{C}+\frac{\partial \mathcal{L}}{\partial \boldsymbol{\Theta}}: \delta \boldsymbol{\Theta}+\frac{1}{\sqrt{\operatorname{det} \boldsymbol{G}}} \frac{\partial \sqrt{\operatorname{det} \boldsymbol{G} \mathcal{L}}}{\partial \boldsymbol{G}}: \delta \boldsymbol{G}\right. \\
\left.+\frac{\partial \mathcal{L}}{\partial \boldsymbol{B}}: \delta \boldsymbol{B}\right) d S d t=\int_{t_{0}}^{t_{1}} \int_{\mathcal{H}}\left(\frac{\partial \mathcal{R}}{\partial \dot{\boldsymbol{G}}}: \delta \boldsymbol{G}+\frac{\partial \mathcal{R}}{\partial \dot{\boldsymbol{B}}}: \delta \boldsymbol{B}\right) d S d t .
\end{array}
$$

Remark 4.1. Note that in taking the variation of the action, the variations of $\boldsymbol{C}$ and $\Theta$ must be such that they satisfy the compatibility equations (3.3). Since the variation of the action is taken by considering the variation of the deformation mapping $\varphi$, the resulting variations of $\boldsymbol{C}$ and $\boldsymbol{\Theta}$ (cf. (4.10) and (4.11)) are trivially compatible, i.e., they trivially satisfy the compatibility equations (3.3). Hence these compatibility equations are not constraints.

If we vary $\epsilon$, for fixed time $t$ and $X \in \mathcal{H}$, the material velocity $\dot{\varphi}_{\epsilon}$ and the unit normal $\mathcal{N} \circ \varphi_{\epsilon}$ lie in $T_{\varphi_{\epsilon, t}(X)} \mathcal{S}$. Therefore, their variations are given by their covariant derivatives along the curve $\varphi_{t, X}$ in $\mathcal{S}$ evaluated at $\epsilon=0$. By the symmetry lemma (see [do Carmo, 1992; Nishikawa, 2002]), we find the variation of the velocity as

$$
\delta \dot{\varphi}=\left.D_{\varphi_{t, X}(\epsilon)} \dot{\varphi}_{\epsilon}\right|_{\epsilon=0}=D_{\varphi_{t, X}(\epsilon)}\left[\left.T_{t} \varphi_{\epsilon, X}\left[\partial_{t}\right]\right|_{\epsilon=0}\right]=\left.D_{\varphi_{X}(t)}\left[T_{\epsilon} \varphi_{t, X}\left[\partial_{\epsilon}\right]\right]\right|_{\epsilon=0}=D_{\varphi_{X}(t)} \delta \varphi=: \frac{D \delta \varphi}{d t} .
$$

Following [Kadianakis and Travlopanos, 2013], the variation of the unit normal vector field is given by

$$
\delta \mathcal{N}=\left.D_{\varphi_{t, X}(\epsilon)} \mathcal{N}_{\epsilon}\right|_{\epsilon=0}=\tilde{\nabla}_{\delta \varphi \|} \mathcal{N}-\left(d\left(\delta \varphi^{n}\right)\right)^{\sharp},
$$

where $\sharp$ denotes the operation of raising indices (sharp operator). In components

$$
\delta \mathcal{N}^{a}=\delta \varphi^{b} \tilde{\nabla}_{\partial_{b}} \mathcal{N}-\frac{\partial\left(\delta \varphi^{n}\right)}{\partial x^{b}} g^{a b} \partial_{a}=-\left(\delta \varphi^{b} \beta^{a}{ }_{b}+\frac{\partial\left(\delta \varphi^{n}\right)}{\partial x^{b}} g^{a b}\right) \partial_{a} .
$$

For any fixed time $t$ and $X \in \mathcal{H}$, the right Cauchy-Green deformation tensor $\boldsymbol{C}_{\epsilon}$ lies in $S^{2} T_{X}^{*} \mathcal{H}$. Therefore, the variation of $\boldsymbol{C}$ is given by its total derivative with respect to $\epsilon$ evaluated at $\epsilon=0$ :

$$
\delta \boldsymbol{C}=\left.\frac{d \boldsymbol{C}_{\epsilon}}{d \epsilon}\right|_{\epsilon=0}=\left.\frac{d}{d \epsilon}\left(\varphi_{\epsilon}^{*} \boldsymbol{g}_{\epsilon}\right)\right|_{\epsilon=0}=\left.\frac{d}{d \epsilon}\left(\varphi^{*} \varphi_{*} \varphi_{\epsilon}^{*} \boldsymbol{g}_{\epsilon}\right)\right|_{\epsilon=0}=\varphi^{*} \boldsymbol{L}_{\delta \varphi} \boldsymbol{g}
$$

Note that [Marsden and Hughes, 1983; Verpoort, 2008; Kadianakis and Travlopanos, 2013] $\boldsymbol{L}_{\delta \varphi} \boldsymbol{g}=\boldsymbol{L}_{\delta \varphi \|} \boldsymbol{g}-$ $2 \delta \varphi^{n} \boldsymbol{\beta}$. Hence, we have

$$
\delta \boldsymbol{C}=\varphi^{*} \boldsymbol{L}_{\delta \varphi \|} \boldsymbol{g}-2 \delta \varphi^{n} \varphi^{*} \boldsymbol{\beta} .
$$

In components, it reads

$$
\delta C_{A B}=F^{a}{ }_{A} g_{a c} \delta \varphi^{c}{ }_{\mid B}+F^{b}{ }_{B} g_{b c} \delta \varphi^{c}{ }_{\mid A}-2 \delta \varphi^{n} F^{a}{ }_{A} F^{b}{ }_{B} \beta_{a b} .
$$

The extrinsic deformation tensor $\Theta_{\epsilon}$ lies in the same space $S^{2} T_{X}^{*} \mathcal{H}$ for fixed time $t$ and $X \in \mathcal{H}$. Hence, the variation of $\Theta$ is given by its total derivative with respect to $\epsilon$ evaluated at $\epsilon=0$ :

$$
\delta \boldsymbol{\Theta}=\left.\frac{d \boldsymbol{\Theta}_{\epsilon}}{d \epsilon}\right|_{\epsilon=0}=\left.\frac{d}{d \epsilon}\left(\varphi_{\epsilon}^{*} \boldsymbol{\beta}_{\epsilon}\right)\right|_{\epsilon=0}=\left.\frac{d}{d \epsilon}\left(\varphi^{*} \varphi_{*} \varphi_{\epsilon}^{*} \boldsymbol{\beta}_{\epsilon}\right)\right|_{\epsilon=0}=\varphi^{*} \boldsymbol{L}_{\delta \varphi} \boldsymbol{\beta} .
$$


The variation in terms of the Lie derivative of the second fundamental form is given by [Verpoort, 2008; Kadianakis and Travlopanos, 2013]

$$
\boldsymbol{L}_{\delta \varphi} \boldsymbol{\beta}=\boldsymbol{L}_{\delta \varphi \|} \boldsymbol{\beta}-\delta \varphi^{n} \mathcal{C}+\operatorname{Hess}_{\delta \varphi^{n}},
$$

where $\mathcal{C}$ denotes the third fundamental form of the surface $\varphi_{t}(\mathcal{H})$ and is defined for $\boldsymbol{x}, \boldsymbol{y} \in \mathfrak{X}\left(\varphi_{t}(\mathcal{H})\right)$ by

$$
\mathcal{C}(\boldsymbol{x}, \boldsymbol{y}):=\boldsymbol{g}\left(\tilde{\nabla}_{\boldsymbol{x}} \boldsymbol{n}, \tilde{\nabla}_{\boldsymbol{y}} \boldsymbol{n}\right)
$$

and $\operatorname{Hess}_{f}$ denotes the Hessian of the scalar-valued function $f$ and is defined for $\boldsymbol{x}, \boldsymbol{y} \in \mathfrak{X}\left(\varphi_{t}(\mathcal{H})\right)$ by

$$
\operatorname{Hess}_{f}(\boldsymbol{x}, \boldsymbol{y}):=\boldsymbol{g}\left(\tilde{\nabla}_{\boldsymbol{x}}(d f)^{\sharp}, \boldsymbol{y}\right) .
$$

Therefore, we have

$$
\delta \boldsymbol{\Theta}=\varphi^{*} \boldsymbol{L}_{\delta \varphi \|} \boldsymbol{\beta}-\delta \varphi^{n} \varphi^{*} \mathcal{C}+\varphi^{*} \operatorname{Hess}_{\delta \varphi^{n}},
$$

or, in components

$$
\delta \Theta_{A B}=F_{A}^{a} F_{B}^{b} \beta_{a b \mid c} \delta \varphi^{c}+F_{A}^{a} \beta_{a c} \delta \varphi^{c}{ }_{\mid B}+F_{B}^{b} \beta_{b c} \delta \varphi^{c}{ }_{\mid A}-\delta \varphi^{n} F_{A}^{a} F_{B}^{b} \beta_{a c} \beta_{b d} g^{c d}+F^{b}{ }_{A}\left(\frac{\partial \delta \varphi^{n}}{\partial x^{b}}\right)_{\mid B} .
$$

The variation of the ambient space metric vanishes identically since it is compatible with the connection, i.e.

$$
\delta \tilde{\boldsymbol{g}} \circ \varphi=D_{\varphi_{t, X}} \tilde{\boldsymbol{g}} \circ \varphi=\tilde{\nabla}_{\delta \varphi} \tilde{\boldsymbol{g}}=\mathbf{0} .
$$

In order to obtain the balance laws, we fix the first and the second fundamental forms, i.e., $\delta \boldsymbol{G}=\mathbf{0}$ and $\delta \boldsymbol{B}=\mathbf{0}$ and vary $\varphi$. Therefore, following (4.7) and by arbitrariness of $\delta \varphi$ and $\nabla^{\mathcal{H}} \delta \varphi^{n}$, we find the following Euler-Lagrange equations ${ }^{8}$

$$
\begin{gathered}
\frac{\partial \mathcal{L}}{\partial \varphi^{a}}-\left(\frac{\partial \mathcal{L}}{\partial \mathcal{N}}\right)_{b} \beta^{b}{ }_{a}-\frac{1}{\sqrt{\operatorname{det} \boldsymbol{G}}} \frac{D}{d t}\left[\sqrt{\operatorname{det} \boldsymbol{G}} \frac{\partial \mathcal{L}}{\partial \dot{\varphi}^{a}}\right]-2\left[\frac{\partial \mathcal{L}}{\partial C_{A B}} F^{b}{ }_{A} g_{a b}\right]_{\mid B} \\
-\left[\frac{\partial \mathcal{L}}{\partial \Theta_{A B}} F^{b}{ }_{A} \beta_{a b}\right]_{\mid B}-\left[\frac{\partial \mathcal{L}}{\partial \Theta_{A B}} F^{b}{ }_{A}\right]_{\mid B} \beta_{a b}=0 \\
\frac{\partial \mathcal{L}}{\partial \varphi^{n}}+\left(\left(\frac{\partial \mathcal{L}}{\partial \mathcal{N}}\right)_{b} g^{a b} F^{-A}{ }_{a}\right)_{\mid A}-\frac{1}{\sqrt{\operatorname{det} \boldsymbol{G}}} \frac{D}{d t}\left[\sqrt{\operatorname{det} \boldsymbol{G}} \frac{\partial \mathcal{L}}{\partial \dot{\varphi}^{n}}\right]-2 \frac{\partial \mathcal{L}}{\partial C_{A B}} F^{b}{ }_{A} F^{a}{ }_{B} \beta_{a b} \\
-\frac{\partial \mathcal{L}}{\partial \Theta_{A B}} F^{b}{ }_{A} F^{a}{ }_{B} \beta_{a c} \beta_{b d} g^{c d}+\left[\left(\frac{\partial \mathcal{L}}{\partial \Theta_{A B}} F^{b}{ }_{A}\right)_{\mid B} F^{-D_{b}}\right]_{\mid D}=0
\end{gathered}
$$

along with the following equations prescribing a vanishing initial velocity vector field and vanishing boundary conditions for the loading on the boundary $\partial \mathcal{H}$

$$
\begin{aligned}
& \left.\frac{\partial \mathcal{L}}{\partial \dot{\varphi}}\right|_{t=t_{0}}=\mathbf{0} \\
& \left(2 \frac{\partial \mathcal{L}}{\partial C_{A B}} F^{c}{ }_{A} g_{a c}+2 \frac{\partial \mathcal{L}}{\partial \Theta_{A B}} F^{c}{ }_{A} \beta_{a c}\right) \mathrm{T}_{B}=0, \\
& {\left[\left(\frac{\partial \mathcal{L}}{\partial \Theta_{A C}} F_{A}^{b}\right)_{\mid C} F^{-B}{ }_{b}+\left(\frac{\partial \mathcal{L}}{\partial \mathcal{N}}\right)_{b} g^{a b} F^{-B}{ }_{a}\right] \mathrm{T}_{B}=0,} \\
& \frac{\partial \mathcal{L}}{\partial \Theta_{A B}} F^{a}{ }_{A} \top_{B}=0,
\end{aligned}
$$

where $\mathbf{T}$ is the outward in-plane vector field normal to the boundary $\partial \mathcal{H}$. 


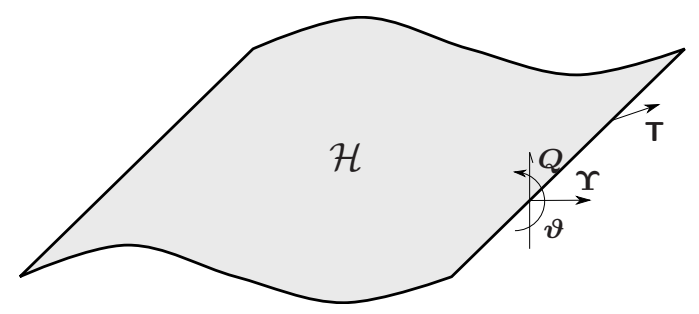

Figure 5: Shell boundary loads: $\boldsymbol{V}, \boldsymbol{\vartheta}$, and $\boldsymbol{Q}$ are the surface traction, the moment, and the shear, respectively. $\mathbf{T}$ denotes the outward in-plane normal.

Remark 4.2. Note that we can modify the Lagrange-d'Alembert's principle in order to prescribe non-vanishing initial and boundary conditions on $\partial \mathcal{H}$. Let $\boldsymbol{V}$ be the boundary surface traction, $\boldsymbol{\vartheta}$ be the boundary moment, $\boldsymbol{Q}$ be the boundary shear, and $\boldsymbol{V}_{t_{0}}$ be the initial velocity vector field (see Figure 5). We write the Lagranged'Alembert's principle as

$$
\begin{array}{r}
\delta S(\varphi, \boldsymbol{G}, \boldsymbol{B})+\int_{t_{0}}^{t_{1}} \int_{\mathcal{H}}\left(\boldsymbol{F}_{\boldsymbol{G}}: \delta \boldsymbol{G}+\boldsymbol{F}_{\boldsymbol{B}}: \delta \boldsymbol{B}\right) d S d t+\int_{t_{0}}^{t_{1}} \int_{\partial \mathcal{H}}\left(\mathrm{J} V^{a} g_{a b} \delta \varphi^{b}+\mathrm{J} \vartheta^{a} \delta \varphi^{n}{ }_{A} F^{-A}{ }_{a}+\mathrm{J} Q \delta \varphi^{n}\right) d L d t \\
+\int_{\mathcal{H}} \rho \boldsymbol{V}_{t_{0}} . \delta \varphi_{t_{0}} d S=0,
\end{array}
$$

and from (4.14) we have

$$
\begin{aligned}
\left.\frac{\partial \mathcal{L}}{\partial \dot{\varphi}}\right|_{t=t_{0}} & =\rho \boldsymbol{V}_{t_{0}}, \\
\left(2 \frac{\partial \mathcal{L}}{\partial C_{A B}} F^{c}{ }_{A} g_{a c}+2 \frac{\partial \mathcal{L}}{\partial \Theta_{A B}} F^{c}{ }_{A} \beta_{a c}\right) \mathrm{T}_{B} & =\mathrm{J} g_{a b} V^{b}, \\
{\left[\left(\frac{\partial \mathcal{L}}{\partial \Theta_{A C}} F^{b}{ }_{A}\right)_{\mid C} F^{-B}{ }_{b}+\left(\frac{\partial \mathcal{L}}{\partial \mathcal{N}}\right)_{b} g^{a b} F^{-B}{ }_{a}\right] \mathrm{T}_{B} } & =\mathrm{J} Q, \\
\frac{\partial \mathcal{L}}{\partial \Theta_{A B}} F^{a}{ }_{A} \mathrm{~T}_{B} & =\mathrm{J} \vartheta^{a} .
\end{aligned}
$$

We introduce the following surface tensors:

Second Piola-Kirchhoff stress tensor: $S=2 \frac{\partial \mathcal{W}}{\partial \boldsymbol{C}}$, in components, $S^{A B}=2 \frac{\partial \mathcal{W}}{\partial C_{A B}}$;

First Piola-Kirchhoff stress tensor: $\boldsymbol{P}=2 \boldsymbol{F} \frac{\partial \mathcal{W}}{\partial \boldsymbol{C}}$, in components, $P^{b B}=2 \frac{\partial \mathcal{W}}{\partial C_{A B}} F_{A}^{b}$;

Cauchy stress tensor: $\boldsymbol{\sigma}=\frac{2}{\mathrm{~J}} \boldsymbol{F} \frac{\partial \mathcal{W}}{\partial \boldsymbol{C}} \boldsymbol{F}^{\top}$, in components, $\sigma^{a b}=\frac{2}{\mathrm{~J}} \frac{\partial \mathcal{W}}{\partial C_{A B}} F^{a}{ }_{A} F^{b}{ }_{B}$;

Material couple stress tensor: $\boldsymbol{M}=\frac{\partial \mathcal{W}}{\partial \Theta}$, in components, $M^{A B}=\frac{\partial \mathcal{W}}{\partial \Theta_{A B}}$;

Two-point couple stress tensor: $\mathcal{M}=\boldsymbol{F} \frac{\partial \mathcal{W}}{\partial \boldsymbol{\Theta}}$, in components, $\mathcal{M}^{b B}=\frac{\partial \mathcal{W}}{\partial \Theta_{A B}} F^{b}{ }_{A}$;

Spatial couple stress tensor: $\boldsymbol{\mu}=\frac{1}{\mathrm{~J}} \boldsymbol{F} \frac{\partial \mathcal{W}}{\partial \boldsymbol{\Theta}} \boldsymbol{F}^{\boldsymbol{\top}}$, in components, $\mu^{a b}=\frac{1}{\mathrm{~J}} \frac{\partial \mathcal{W}}{\partial \Theta_{A B}} F^{a}{ }_{A} F^{b}{ }_{B}$.

We further introduce the following notations for the external loads

$$
\begin{aligned}
& \text { External body forces : } \mathcal{B}=-\frac{1}{\rho} \frac{\partial \mathcal{V}}{\partial \varphi}, \\
& \text { External body moments : } \mathcal{L}=-\frac{1}{\rho} \frac{\partial \mathcal{V}}{\partial \mathcal{N}} .
\end{aligned}
$$

\footnotetext{
${ }^{8}$ We denote by $F^{-A_{a}}$ the components of $\boldsymbol{F}^{-1}$, the inverse of $\boldsymbol{F}$. See Appendix 6 for the details of the derivation of (4.13) and (4.14) following (4.7).
} 
Recalling the balance of mass (4.3), we have for the Lagrangian density (4.6)

$$
\frac{1}{\sqrt{\operatorname{det} \boldsymbol{G}}} \frac{D}{d t}\left[\sqrt{\operatorname{det} \boldsymbol{G}} \frac{\partial \mathcal{L}}{\partial \dot{\varphi}}\right]=\rho \tilde{\boldsymbol{g}} \boldsymbol{A}+S_{m} \tilde{\boldsymbol{g}} \boldsymbol{V}
$$

Therefore, the Euler-Lagrange equations (4.13) read

$$
\begin{aligned}
\left(P^{a B}+\beta^{a}{ }_{b} \mathcal{M}^{b B}\right)_{\mid B}+\beta^{a}{ }_{b}\left(\mathcal{M}^{b B}{ }_{\mid B}\right)+\rho \mathcal{B}^{a}-\rho \beta^{a}{ }_{b} \mathcal{L}^{b}-S_{m} \dot{\varphi}^{a} & =\rho A^{a}, \\
\left(P^{a B}+\beta^{a}{ }_{b} \mathcal{M}^{b B}\right) F^{c}{ }_{B} \beta_{a c}-\left(\mathcal{M}^{b B}{ }_{\mid B} F^{-D}{ }_{b}\right)_{\mid D}+\rho \mathcal{B}^{n}+\left(\rho \mathcal{L}^{a} F^{-A}{ }_{a}\right)_{\mid A}-S_{m} \dot{\varphi}^{n} & =\rho A^{n},
\end{aligned}
$$

and the vanishing initial and boundary conditions

$$
\begin{aligned}
\left.\boldsymbol{V}\right|_{t=t_{0}} & =\mathbf{0} \\
\left(g_{a b} P^{b B}+2 \beta_{a b} \mathcal{M}^{b B}\right) \mathrm{T}_{B} & =0 \\
{\left[\mathcal{M}^{b C}{ }_{\mid C} F^{-B}{ }_{b}+\rho \mathcal{L}^{a} F^{-B}{ }_{a}\right] \mathrm{T}_{B} } & =0 \\
\mathcal{M}^{a B} \mathrm{~T}_{B} & =0 .
\end{aligned}
$$

We apply the Piola transform ${ }^{9}$ to (4.17) and (4.18) and obtain the spatial version of the balance of linear momenta as

$$
\begin{aligned}
& \left(\sigma^{a c}+\beta^{a}{ }_{b} \mu^{b c}\right)_{\mid c}+\beta^{a}{ }_{b} \mu^{b c}{ }_{\mid c}+\varrho \mathcal{B}^{a}-\varrho \beta^{a}{ }_{b} \mathcal{L}^{b}-s_{m} \dot{\varphi}^{a}=\varrho A^{a}, \\
& \left(\sigma^{a c}+\beta^{a}{ }_{b} \mu^{b c}\right) \beta_{a c}-\mu^{a b}{ }_{\mid a b}+\varrho \mathcal{B}^{n}+\left(\varrho \mathcal{L}^{a}\right)_{\mid a}-s_{m} \dot{\varphi}^{n}=\varrho A^{n},
\end{aligned}
$$

and the vanishing initial and boundary conditions

$$
\begin{aligned}
\left.\boldsymbol{V}\right|_{t=t_{0}} & =\mathbf{0}, \\
\left(\sigma^{a c}+2 \beta^{a}{ }_{b} \mu^{b c}\right) \mathbf{t}_{c} & =0, \\
\left(\mu^{a b}{ }_{\mid b}+\varrho \mathcal{L}^{a}\right) \mathbf{t}_{a} & =0, \\
\mu^{a b} \mathrm{t}_{b} & =0,
\end{aligned}
$$

where $\mathbf{t}$ is the outward in-plane vector field normal to the boundary $\partial \varphi_{t}(\mathcal{H})$. By pulling back the system of equations (4.19) with the mapping $\varphi$, we obtain the Euler-Lagrange equations in the convected manifold $(\mathcal{H}, \boldsymbol{C})^{10}$ in terms of the convected stress tensor $\boldsymbol{\Sigma}=\varphi^{*} \boldsymbol{\sigma}=\boldsymbol{S} / \mathrm{J}$ and the convected couple stress tensor $\boldsymbol{\Lambda}=\varphi^{*} \boldsymbol{\mu}=\boldsymbol{M} / \mathrm{J}$. If we denote by a double stroke $\|$ the covariant derivative corresponding to the Levi-Civita connection of $(\mathcal{H}, \boldsymbol{C})$, the convected Euler-Lagrange equation read ${ }^{11}$

$$
\begin{gathered}
\left(\Sigma^{A B}+C^{-A C} \Theta_{C D} \Lambda^{D B}\right)_{\| B}+C^{-A C} \Theta_{C D} \Lambda^{D B} \| B_{\| B}+\varrho F^{-A}{ }_{a} \mathcal{B}^{a} \\
-\varrho C^{-A C} \Theta_{C D} F^{-D}{ }_{a} \mathcal{L}^{a}-s_{m} F^{-A}{ }_{a} \dot{\varphi}^{a}=\varrho F^{-A}{ }_{a} A^{a}, \\
\left(\Sigma^{A B}+C^{-A C} \Theta_{C D} \Lambda^{D B}\right) \Theta_{A B}-\Lambda^{A B}{ }_{\| A B}+\varrho \mathcal{B}^{n}+\left(\varrho \mathcal{L}^{a} F^{-A}{ }_{a}\right)_{\| A}-s_{m} \dot{\varphi}^{n}=\varrho A^{n},
\end{gathered}
$$

and the vanishing initial and boundary conditions

$$
\begin{aligned}
\left.\boldsymbol{V}\right|_{t=t_{0}} & =\mathbf{0}, \\
\left(\Sigma^{A B}+2 C^{-A C} \Theta_{C D} \Lambda^{D B}\right) \mathrm{T}_{B} & =0, \\
\left(\Lambda^{A B}{ }_{\| B}+\varrho F^{-A}{ }_{a} \mathcal{L}^{a}\right) \mathrm{T}_{A} & =0, \\
\Lambda^{A B} \mathrm{~T}_{B} & =0 .
\end{aligned}
$$

\footnotetext{
${ }^{9}$ Recall the Piola identity $\left(J F^{-A_{a}}\right)_{\mid A}=0$.

${ }^{10}$ We define the convected manifold to be the material manifold $\mathcal{H}$ equipped with the right Cauchy-Green deformation tensor $\boldsymbol{C}$.

${ }^{11}$ The components of $\boldsymbol{C}^{-1}$, the inverse of $\boldsymbol{C}$, are denoted by $C^{-A B}$.
} 
Recall that in terms of the deformation mapping $\varphi: \mathcal{H} \rightarrow \mathbb{R}^{3}$, we can write the components of $\boldsymbol{C}$ and $\boldsymbol{\Theta}$ in a local chart $\{X, Y\}$ of $\mathcal{H}$ as follow

$$
\begin{aligned}
& C_{A B}=\varphi, A \cdot \varphi, B, \\
& \Theta_{A B}=\varphi, A B \cdot \frac{\varphi, X \times \varphi, Y}{\left\|\varphi, X \times \varphi_{, Y}\right\|} .
\end{aligned}
$$

where $\cdot, \times$, and $\|\cdot\|$, respectively, denote the dot product, the cross product, and the standard norm in $\mathbb{R}^{3}$.

Given a constitutive relation, the stress and the couple stress tensors can be written in terms of the first and the second fundamental forms of the deformed surface. On the other hand, the first and the second fundamental forms of the deformed surface can be written in terms of the motion $\varphi$ such that the compatibility equations (3.3) are trivially satisfied. Therefore, the system of equations (4.17) (or (4.19), or (4.21)) is a set of three equations for three unknowns (the three components of the motion), and together with the initial and boundary conditions (4.18) (or (4.20), or (4.22)), they form the complete set of governing equations for the morphoelastic shell problem.

Remark 4.3. Note that both systems of equations (4.17) and (4.19) reduce to the elastic shell equilibrium equations for a zero-acceleration motion in the absence of growth $\left(s_{m}=0\right)$ and dissipation $(\mathcal{R}=0)$. See $[$ Chien, 1943] (Equations (2.8)), [Sanders Jr, 1961] (Equations (55) and (56)), [Naghdi, 1963] (Equations (5.36)), and [Koiter, 1966] (Equations (6.3) and (6.4)) where the shell problem is described by a system of three equations involving six stress and couple stress components. Note that an alternative description is provided by a system of six equations involving ten stress and couple stress components, see [Green and Zerna, 1950] (Equations (3.6) and (3.11)) and [Ericksen and Truesdell, 1958] (Equations (26.6), (26.7) and (26.10)).

Remark 4.4. Following the definitions of the surface tensors and based on the symmetry of the right CauchyGreen tensor and the extrinsic deformation tensor, we have the following symmetries for the stress tensors, which are the local forms of the balance of angular momenta

$$
\begin{array}{rlrlrl}
\boldsymbol{S}^{\top} & =\boldsymbol{S}, & \boldsymbol{\Sigma}^{\top}=\boldsymbol{\Sigma}, & \boldsymbol{P} \boldsymbol{F}^{\top}=\boldsymbol{P}^{\top} \boldsymbol{F}, & \boldsymbol{\sigma}^{\top}=\boldsymbol{\sigma} \\
\boldsymbol{M}^{\top}=\boldsymbol{M}, & \boldsymbol{\Lambda}^{\top}=\boldsymbol{\Lambda}, & \mathcal{M} \boldsymbol{F}^{\top}=\mathcal{M}^{\top} \boldsymbol{F}, & \boldsymbol{\mu}^{\top}=\boldsymbol{\mu} .
\end{array}
$$

\subsection{Kinetic equations of growth}

To obtain the kinetic equation governing the evolution of growth, we fix the motion, i.e. $\delta \varphi=0$, and vary the first and the second fundamental forms. From the arbitrariness of $\delta \boldsymbol{G}$ and $\delta \boldsymbol{B}$, we obtain from (4.7) the following kinetic equations for the evolution of the first and the second fundamental forms of $\mathcal{H}$ :

$$
\begin{aligned}
& \frac{1}{\sqrt{\operatorname{det} \boldsymbol{G}}} \frac{\partial(\sqrt{\operatorname{det} \boldsymbol{G}} \mathcal{L})}{\partial \boldsymbol{G}}=\frac{\partial \mathcal{R}}{\partial \dot{\boldsymbol{G}}}, \\
& \frac{\partial \mathcal{L}}{\partial \boldsymbol{B}}=\frac{\partial \mathcal{R}}{\partial \dot{\boldsymbol{B}}} .
\end{aligned}
$$

Therefore, we find the following for the Lagrangian density (4.6)

$$
\begin{aligned}
& \frac{\partial \mathcal{R}}{\partial \dot{\boldsymbol{G}}}=\frac{1}{2}\left(\frac{1}{2} \rho \tilde{\boldsymbol{g}}(\dot{\varphi}, \dot{\varphi})-\mathcal{W}-\mathcal{V}\right) \boldsymbol{G}^{\sharp}-\frac{\partial \mathcal{W}}{\partial \boldsymbol{G}}, \\
& \frac{\partial \mathcal{R}}{\partial \dot{\boldsymbol{B}}}=-\frac{\partial \mathcal{W}}{\partial \boldsymbol{B}} .
\end{aligned}
$$

Assuming the existence of a Rayleigh potential $\mathcal{R}=\mathcal{R}(\dot{\boldsymbol{G}}, \dot{\boldsymbol{B}}, \boldsymbol{G})$, we introduce a variational characterization for the variation of energy in the shell due to growth. The system of equations (4.25) provide a coupling of the rate of change of the first and the second fundamental forms of $\mathcal{H}$ with its current state of deformation through the elastic energy density $\mathcal{W}$ of the material. Therefore, the evolution of the geometry of the shell, i.e., the growth of the morphoelastic shell, is governed by (4.25). 
Remark 4.5. Yavari [2010] discussed the kinetic equation for the evolving metric in the case of bulk growth for three-dimensional nonlinear elasticity. Note, however, that there was a missing term in equation (2.179), which should be corrected to read

$$
\delta \int_{t_{0}}^{t_{1}} \int_{\mathcal{B}} \mathcal{L} d V d t=\int_{t_{0}}^{t_{1}} \int_{\mathcal{B}}\left[\delta \mathcal{L}+\frac{1}{2} \mathcal{L} \operatorname{tr}(\delta \boldsymbol{G})\right] d V d t
$$

The kinetic equation (2.181) in [Yavari, 2010] should also be corrected to read

$$
\frac{\partial \mathcal{R}}{\partial \dot{\boldsymbol{G}}}=\frac{\partial \mathcal{L}}{\partial \boldsymbol{G}}+\frac{1}{2} \mathcal{L} \boldsymbol{G}^{\sharp} .
$$

Ignoring inertial forces and in the absence of body forces, (4.26) reads

$$
\frac{\partial \mathcal{R}}{\partial \dot{\boldsymbol{G}}}=-\frac{\partial \mathcal{W}}{\partial \boldsymbol{G}}-\frac{1}{2} \mathcal{W} \boldsymbol{G}^{\sharp}
$$

Following the material covariance of nonlinear elasticity, Lu and Papadopoulos [2000]; Yavari et al. [2006] proved the following relation:

$$
\frac{1}{2} \boldsymbol{S}_{o} \boldsymbol{G}=\frac{\partial \mathcal{W}}{\partial \boldsymbol{G}} \boldsymbol{G}+\frac{\partial \mathcal{W}}{\partial \boldsymbol{C}} \boldsymbol{C},
$$

where $\boldsymbol{S}_{o}$ is a stress-like tensor conjugate to $\dot{\boldsymbol{G}} . \boldsymbol{S}_{o}$ is associated with the material evolution and is a measure of anisotropy of the medium: $\boldsymbol{S}_{o}=\mathbf{0}$ for an isotropic material. Therefore, (4.26) can be rewritten as

$$
\frac{\partial \mathcal{R}}{\partial \dot{\boldsymbol{G}}} \boldsymbol{G}=-\frac{1}{2} \mathcal{W} \boldsymbol{G}^{\sharp} \boldsymbol{G}+\frac{1}{2} \boldsymbol{S} \boldsymbol{C}-\frac{1}{2} \boldsymbol{S}_{o} \boldsymbol{G} .
$$

In the context of the multiplicative decomposition of the deformation gradient $\boldsymbol{F}=\boldsymbol{F}_{e} \boldsymbol{F}_{g}$, the kinetic equation coupling the evolution of growth and stress is written in terms of the growth tensor $\boldsymbol{F}_{g}$. Fusi et al. [2006] derived it using the so-called principle of maximum entropy production rate, and Ambrosi and Guana [2007]; Olsson and Klarbring [2008] used the Clausius-Duhem inequality. Note that these equations are both equivalent and similar in form to (4.27).

Example 4.1. As an example, we consider the following Rayleigh potential

$$
\mathcal{R}(\dot{\boldsymbol{G}}, \dot{\boldsymbol{B}}, \boldsymbol{G})=\alpha_{1} \operatorname{tr}(\dot{\boldsymbol{G}})+\alpha_{2} \operatorname{tr}\left(\dot{\boldsymbol{G}}^{2}\right)+\beta_{1} \operatorname{tr}(\dot{\boldsymbol{B}})+\beta_{2} \operatorname{tr}\left(\dot{\boldsymbol{B}}^{2}\right) .
$$

In components

$$
\mathcal{R}(\dot{\boldsymbol{G}}, \dot{\boldsymbol{B}}, \boldsymbol{G})=\alpha_{1} \dot{G}_{A B} G^{A B}+\alpha_{2} \dot{G}_{A B} \dot{G}_{C D} G^{C A} G^{D B}+\beta_{1} \dot{B}_{A B} G^{A B}+\beta_{2} \dot{B}_{A B} \dot{B}_{C D} G^{C A} G^{D B} .
$$

Therefore, if we assume a static shell in the absence of body forces and moments, the kinetic equations (4.25) read

$$
\begin{aligned}
\dot{\boldsymbol{G}} & =-\frac{\alpha_{1}}{2 \alpha_{2}} \boldsymbol{G}-\frac{1}{4 \alpha_{2}} \mathcal{W} \boldsymbol{G}-\frac{1}{2 \alpha_{2}} \boldsymbol{G} \frac{\partial \mathcal{W}}{\partial \boldsymbol{G}} \boldsymbol{G}, \\
\dot{\boldsymbol{B}} & =-\frac{\beta_{1}}{2 \beta_{2}} \boldsymbol{G}-\frac{1}{2 \beta_{2}} \boldsymbol{G} \frac{\partial \mathcal{W}}{\partial \boldsymbol{B}} \boldsymbol{G} .
\end{aligned}
$$

In components

$$
\begin{aligned}
\dot{G}_{A B} & =-\frac{\alpha_{1}}{2 \alpha_{2}} G_{A B}-\frac{1}{4 \alpha_{2}} \mathcal{W} G_{A B}-\frac{1}{2 \alpha_{2}} G_{A C} \frac{\partial \mathcal{W}}{\partial G_{D C}} G_{D B}, \\
\dot{B}_{A B} & =-\frac{\beta_{1}}{2 \beta_{2}} G_{A B}-\frac{1}{2 \beta_{2}} G_{A C} \frac{\partial \mathcal{W}}{\partial B_{D C}} G_{D B} .
\end{aligned}
$$


We assume a Saint Venant-Kirchhoff constitutive model, for which the strain energy density $\mathcal{W}$ is given by ${ }^{12}$

$$
\begin{aligned}
\mathcal{W} & =\frac{h}{4}\left\{\mu \operatorname{tr}\left[(\boldsymbol{C}-\boldsymbol{G})^{2}\right]+\frac{\mu \lambda}{2 \mu+\lambda}[\operatorname{tr}(\boldsymbol{C}-\boldsymbol{G})]^{2}\right\}+\frac{h^{3}}{12}\left\{\mu \operatorname{tr}\left[(\boldsymbol{\Theta}-\boldsymbol{B})^{2}\right]+\frac{\mu \lambda}{2 \mu+\lambda}[\operatorname{tr}(\boldsymbol{\Theta}-\boldsymbol{B})]^{2}\right\} \\
& =\frac{E h}{8(1+\nu)}\left\{\operatorname{tr}\left[(\boldsymbol{C}-\boldsymbol{G})^{2}\right]+\frac{\nu}{1-\nu}[\operatorname{tr}(\boldsymbol{C}-\boldsymbol{G})]^{2}\right\}+\frac{E h^{3}}{24(1+\nu)}\left\{\operatorname{tr}\left[(\boldsymbol{\Theta}-\boldsymbol{B})^{2}\right]+\frac{\nu}{1-\nu}[\operatorname{tr}(\boldsymbol{\Theta}-\boldsymbol{B})]^{2}\right\},
\end{aligned}
$$

where $\lambda$ and $\mu$ are respectively Lamé's first and second parameters, $E$ is the Young's modulus, and $\nu$ is Poisson's ratio. Therefore, for a Saint Venant-Kirchhoff constitutive model, the kinetic equations (4.29) read

$$
\begin{aligned}
\dot{G}_{A B}= & -\frac{\alpha_{1}}{2 \alpha_{2}} G_{A B}-\frac{h}{16 \alpha_{2}}\left[\mu\left(C_{K L}-G_{K L}\right)\left(C^{K L}-G^{K L}\right)+\frac{\mu \lambda}{2 \mu+\lambda}\left(C^{K}{ }_{K}-2\right)^{2}\right] G_{A B} \\
& -\frac{h^{3}}{48 \alpha_{2}}\left[\mu\left(\Theta_{K L}-B_{K L}\right)\left(\Theta^{K L}-B^{K L}\right)+\frac{\mu \lambda}{2 \mu+\lambda}\left(\Theta^{K}{ }_{K}-B^{K}{ }_{K}\right)^{2}\right] G_{A B} \\
& +\frac{h}{4 \alpha_{2}}\left[\mu C_{A K}\left(C_{L B}-G_{L B}\right) G^{K L}+\frac{\mu \lambda}{2 \mu+\lambda}\left(C^{K}{ }_{K}-2\right) C_{A B}\right] \\
& +\frac{h^{3}}{12 \alpha_{2}}\left[\mu\left(\Theta_{A K}-B_{A K}\right)\left(\Theta_{L B}-B_{L B}\right) G^{K L}+\frac{\mu \lambda}{2 \mu+\lambda}\left(\Theta^{K}{ }_{K}-B^{K}{ }_{K}\right)\left(\Theta_{A B}-B_{A B}\right)\right], \\
\dot{B}_{A B}= & -\frac{\beta_{1}}{2 \beta_{2}} G_{A B}+\frac{h^{3}}{12 \beta_{2}}\left[\mu\left(\Theta_{A B}-B_{A B}\right)+\frac{\mu \lambda}{2 \mu+\lambda}\left(\Theta^{K}{ }_{K}-B^{K}{ }_{K}\right) G_{A B}\right] .
\end{aligned}
$$

\section{$5 \quad$ Examples}

As applications of the proposed geometric theory of morphoelastic shells, we study in this section some examples of growth and the induced residual stress fields. We look at the stress-free growth of an initially planar sheet, and study the residual stress and geometry evolution of a morphoelastic infinitely long circular cylindrical shell subject to an internal pressure and a morphoelastic initially planar circular disk.

\subsection{Stress-free growth fields for an initially flat simply-connected shell}

We consider an initially flat thin shell $\mathcal{B}$ such that its mid-surface $\mathcal{H}$ is simply-connected. Let $(X, Y, Z)$ be the standard coordinate chart for $\mathbb{R}^{3}$ such that the hyperplane $X^{3}=0$ contains $\mathcal{H}$. We assume that the morphoelastic shell is undergoing a growth field that is modeled by the following evolving metric for $\mathcal{B}$ :

$$
\overline{\boldsymbol{G}}=\left(\begin{array}{ccc}
e^{2 \bar{\omega}_{X}(X, Y, Z, t)} & 0 & 0 \\
0 & e^{2 \bar{\omega}_{Y}(X, Y, Z, t)} & 0 \\
0 & 0 & 1
\end{array}\right),
$$

which corresponds to the following evolving first and second fundamental forms for the mid-surface $\mathcal{H}$ :

$$
\begin{gathered}
\boldsymbol{G}=\left(\begin{array}{cc}
e^{2 \omega_{X}(X, Y, t)} & 0 \\
0 & e^{2 \omega_{Y}(X, Y, t)}
\end{array}\right) \\
\boldsymbol{B}=\left(\begin{array}{cc}
-K_{X}(X, Y, t) e^{2 \omega_{X}(X, Y, t)} & 0 \\
0 & -K_{Y}(X, Y, t) e^{2 \omega_{Y}(X, Y, t)}
\end{array}\right),
\end{gathered}
$$

where $\omega_{A}(X, Y, t)=\bar{\omega}_{A}(X, Y, 0, t)$ and $K_{A}(X, Y, t)=\frac{\partial \bar{\omega}_{A}}{\partial Z}(X, Y, 0, t)$ for $A=X, Y$. Following $\S 3.3$, the growth of a simply-connected shell is stress-free if and only if the relations (3.5) hold. In the case of an initially flat

\footnotetext{
${ }^{12}$ For details on the derivation of the Saint Venant-Kirchhoff shell model, see [Fox et al., 1993; Le Dret and Raoult, 1993; Lods and Miara, 1995; Miara, 1998; Lods and Miara, 1998; Friesecke et al., 2002a,b, 2003].
} 


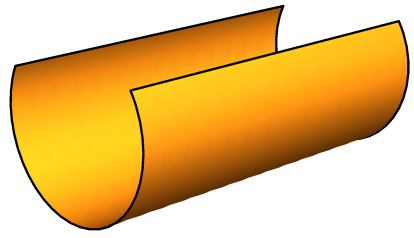

(a)

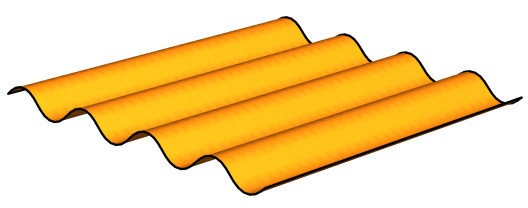

(b)

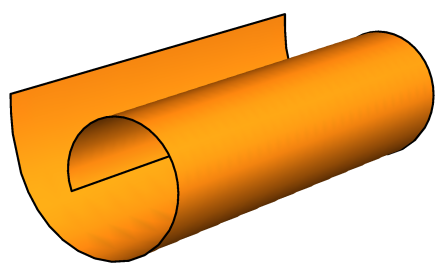

(c)

Figure 6: Visualization of a few stress-free material evolutions of an initially planar sheet with the prescribed evolving fundamental forms such that the in-plane growth is uniform, i.e., $\omega_{A}=\omega_{A}(t)$ for $A=X, Y$, the Gaussian curvature is vanishing, i.e., $K_{X} K_{Y}=0$, and the non-zero principal curvature is such that $K_{X}=K_{X}(X, t)$ or $K_{Y}=K_{Y}(Y, t)$. We assume for these figures that $K_{Y}=0$ and (a) $K_{X}=K_{X}(t)$ to grow to a cylindrical portion, (b) $K_{X}(X, t)=k_{1}(t) \sin \left(k_{2}(t) X\right)$, where $k_{1}=k_{1}(t)$ and $k_{2}=k_{2}(t)$ are some arbitrary functions of time resulting in a sheet with sinusoidal rippling, and $(c) K_{X}(X, t)=k(t) \sqrt{X}$, for $X>0$, where $k=k(t)$ is some arbitrary function of time.

morphoelastic simply-connected shell, the growth is stress-free if and only if

$$
\begin{aligned}
e^{2 \omega_{X}}\left[\left(\frac{\partial \omega_{Y}}{\partial Y}-\frac{\partial \omega_{X}}{\partial Y}\right) \frac{\partial \omega_{X}}{\partial Y}-\frac{\partial^{2} \omega_{X}}{\partial Y^{2}}\right]+e^{2 \omega_{Y}}\left[\left(\frac{\partial \omega_{X}}{\partial X}-\frac{\partial \omega_{Y}}{\partial X}\right) \frac{\partial \omega_{Y}}{\partial X}-\frac{\partial^{2} \omega_{Y}}{\partial X^{2}}\right]=K_{X} K_{Y} e^{2 \omega_{X}} e^{2 \omega_{Y}}, \\
\frac{\partial K_{X}}{\partial Y}=\left(K_{Y}-K_{X}\right) \frac{\partial \omega_{X}}{\partial Y} \\
\frac{\partial K_{Y}}{\partial X}=\left(K_{X}-K_{Y}\right) \frac{\partial \omega_{Y}}{\partial X}
\end{aligned}
$$

Now we consider the following simplifying assumptions:

- If we assume that the in-plane growth is uniform, i.e., $\omega_{A}=\omega_{A}(t)$ for $A=X, Y$, we find that the growth is stress-free if and only if $K_{X}=K_{X}(X, t), K_{Y}=K_{Y}(Y, t)$, and $K_{X} K_{Y}=0$. This case includes the stress-free growth of a planar sheet into a cylindrical portion. See Figure 6 for examples of evolutions of planar sheets into flat surfaces with stress-free growth.

- If we assume that the evolving curvatures $K_{X}$ and $K_{Y}$ are uniform, i.e., $K_{A}=K_{A}(t)$ for $A=X$, $Y$, we distinguish the following cases:

- If $K_{X} \neq K_{Y}$, then the growth is stress-free if and only if $\omega_{X}$ and $\omega_{Y}$ are uniform and $K_{X} K_{Y}=0$. This is precisely the case of a planar sheet evolving to a cylindrical portion with a stress-free growth (see Figure 6a).

- If $K=K_{X}=K_{Y}$, then the growth is stress-free if and only if

$$
e^{2 \omega_{X}}\left[\left(\frac{\partial \omega_{Y}}{\partial Y}-\frac{\partial \omega_{X}}{\partial Y}\right) \frac{\partial \omega_{X}}{\partial Y}-\frac{\partial^{2} \omega_{X}}{\partial Y^{2}}\right]+e^{2 \omega_{Y}}\left[\left(\frac{\partial \omega_{X}}{\partial X}-\frac{\partial \omega_{Y}}{\partial X}\right) \frac{\partial \omega_{Y}}{\partial X}-\frac{\partial^{2} \omega_{Y}}{\partial X^{2}}\right]=K^{2} e^{2 \omega_{X}} e^{2 \omega_{Y}}
$$

- If we assume that the in-plane growth is isotropic, i.e., $\omega=\omega_{X}=\omega_{Y}$, we distinguish the following cases:

- If $K=K_{X}=K_{Y}$, then the growth is stress-free if and only if $K$ is uniform and $\frac{\partial^{2} \omega}{\partial Y^{2}}+\frac{\partial^{2} \omega}{\partial X^{2}}=-K^{2} e^{2 \omega}$. In particular, if $K_{X}=K_{Y}=0$, then the growth is stress-free if and only if $\omega$ is harmonic. See Example 5.1 and Figure 7 for examples of such a stress-free growth.

- If $K_{X} \neq K_{Y}$, then the growth is stress-free if and only if

$$
\begin{gathered}
\frac{\partial^{2} \omega}{\partial Y^{2}}+\frac{\partial^{2} \omega}{\partial X^{2}}=-K_{X} K_{Y} e^{2 \omega} \\
\frac{\partial K_{X}}{\partial Y}=\left(K_{Y}-K_{X}\right) \frac{\partial \omega}{\partial Y} \\
\frac{\partial K_{Y}}{\partial X}=\left(K_{X}-K_{Y}\right) \frac{\partial \omega}{\partial X}
\end{gathered}
$$

See Example 5.2 and Figure 8 for examples of such a stress-free growth assuming that $K_{X}=-K_{Y}$. 
a)
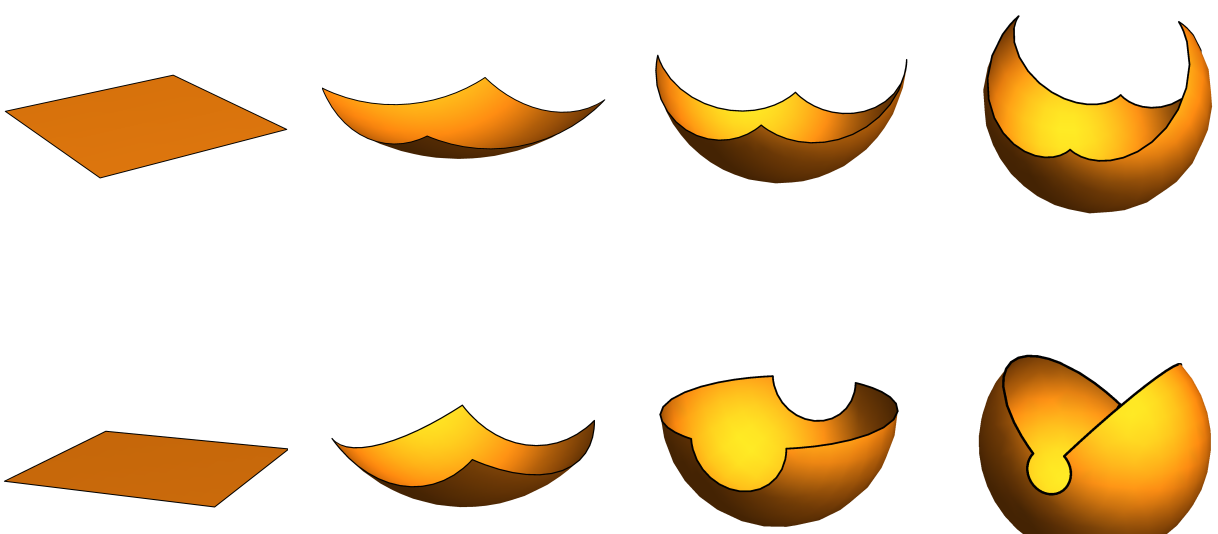

b)

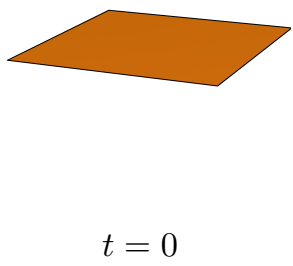

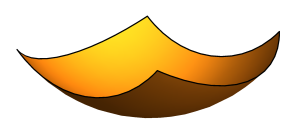

$$
t=\frac{\pi}{4} \tau
$$

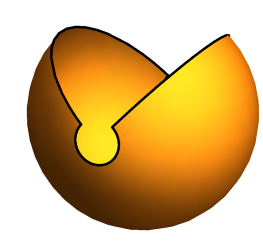

$t=\frac{3 \pi}{4} \tau$
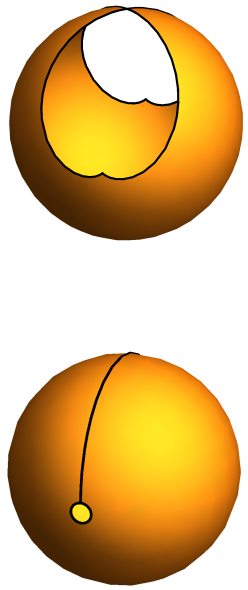

$t=\pi \tau$

Figure 7: Example 5.1: Visualization of the stress-free material evolution of an initially planar sheet with the prescribed evolving fundamental forms (5.6) and (5.7), shown, respectively, in a) and b), at different times. Note that the change of shape of the shell is due to growth and not stretch; such an evolution is stress-free.

Example 5.1. In this example, we consider a morphoelastic initially planar square sheet in the $X Y$-plane such that center of the shell coincides with the origin of the coordinate system and the sides of the shell are parallel to the $X$ and $Y$ axes. We assume that both the in-plane and the out-of-plane growths are isotropic, i.e., $\omega=\omega_{X}=\omega_{Y}$, and $K=K_{X}=K_{Y}$. Therefore, the growth is stress-free if and only if $K=K(t)$ is a uniform arbitrary function of time and $\omega$ is such that

$$
\frac{\partial^{2} \omega}{\partial Y^{2}}+\frac{\partial^{2} \omega}{\partial X^{2}}=-K^{2} e^{2 \omega}
$$

Following Polyanin and Zaitsev [2004], a solution of (5.4) is given by

$$
\omega(X, Y, t)=\frac{1}{2} \ln \left(\frac{A^{2}(t)+B^{2}(t)}{K^{2}(t) \cosh ^{2}[C(t)+A(t) X+B(t) Y]}\right),
$$

for some arbitrary functions of time $A=A(t), B=B(t), C=C(t)$, and $K=K(t)$. Therefore, the first and the second fundamental forms read

$$
\begin{aligned}
& \boldsymbol{G}=\frac{A^{2}(t)+B^{2}(t)}{K^{2}(t) \cosh ^{2}[C(t)+A(t) X+B(t) Y]}\left(\begin{array}{ll}
1 & 0 \\
0 & 1
\end{array}\right), \\
& \boldsymbol{B}=-\frac{A^{2}(t)+B^{2}(t)}{K(t) \cosh ^{2}[C(t)+A(t) X+B(t) Y]}\left(\begin{array}{ll}
1 & 0 \\
0 & 1
\end{array}\right) .
\end{aligned}
$$

It is readily seen that every point of the surface is an umbilical point (the principal curvatures are equal to $K(t)$ ). Therefore, at a given time $t$, we have a surface of constant non-negative curvature $K^{2}(t)$, and hence it is either a planar $(K=0)$ or a spherical $(K>0)$ surface of radius $1 / K(t)$ (see Figure 7 ).

The functions $A=A(t), B=B(t), C=C(t)$, and $K=K(t)$ define the time evolution of the first and the second fundamental forms. Given a constitutive equation for the material, their evolution can subsequently be obtained from the kinetic equations (4.25) governing the evolution of growth. As an example, and for the purpose of illustrating the non-trivial evolution of the initially planar shell as a result of a stress-free growth, we consider the following cases:

- We assume that $A(t)=t / \tau, B(t)=t / \tau, C(t)=0$ and $K_{0}(t)=\sqrt{2} t / \tau$, where $\tau$ is some growth characteristic time. It follows that $\omega(X, Y, t)=-\ln \{\cosh [(X+Y) t / \tau]\}$, such that at $t=0$ they satisfy 
$\omega(X, Y, 0)=0$ and $K(0)=0$. Therefore, we have the following evolving first and second fundamental forms:

$$
\boldsymbol{G}=\frac{1}{\cosh ^{2}[(X+Y) t / \tau]}\left(\begin{array}{ll}
1 & 0 \\
0 & 1
\end{array}\right), \quad \boldsymbol{B}=-\frac{\sqrt{2} t / \tau}{\cosh ^{2}[(X+Y) t / \tau]}\left(\begin{array}{ll}
1 & 0 \\
0 & 1
\end{array}\right) .
$$

- We assume that $A(t)=2 t / \tau, B(t)=0, C(t)=0$ and $K_{0}(t)=2 t / \tau$. It follows that $\omega(X, Y, t)=$ $-\ln \{\cosh [2 X t / \tau]\}$, such that at $t=0$ they satisfy $\omega(X, Y, 0)=0$ and $K(X, Y, 0)=0$. Therefore, we have the following evolving first and second fundamental forms:

$$
\boldsymbol{G}=\frac{1}{\cosh ^{2}[2 X t / \tau]}\left(\begin{array}{ll}
1 & 0 \\
0 & 1
\end{array}\right), \quad \boldsymbol{B}=-\frac{2 t / \tau}{\cosh ^{2}[2 X t / \tau]}\left(\begin{array}{ll}
1 & 0 \\
0 & 1
\end{array}\right) .
$$

We visualize in Figure 7, the evolution of the initially planar sheet with the prescribed fundamental forms (5.6) and (5.7).

Example 5.2. In this example, we consider a morphoelastic initially flat square sheet in the $X Y$-plane such that the center of the shell coincides with the origin of the coordinate system and the sides of the shell are parallel to the $X$ and $Y$ axes. We assume that the in-plane growth is isotropic, i.e., $\omega=\omega_{X}=\omega_{Y}$, and assume that $K=K_{X}=-K_{Y} \neq 0$. We look for $\omega$ and $K$ such that the growth is stress-free, i.e., such that

$$
\begin{gathered}
\frac{\partial^{2} \omega}{\partial Y^{2}}+\frac{\partial^{2} \omega}{\partial X^{2}}=K^{2} e^{2 \omega}, \\
\frac{\partial K}{\partial Y}=-2 K \frac{\partial \omega}{\partial Y}, \\
\frac{\partial K}{\partial X}=-2 K \frac{\partial \omega}{\partial X} .
\end{gathered}
$$

It follows from (5.8b) and (5.8c) that $K(X, Y, t)=K_{o}(t) e^{-2 \omega(X, Y, t)}$ for some arbitrary function of time $K_{o}=$ $K_{o}(t)$. Therefore, (5.8a) now reads

$$
\frac{\partial^{2} \omega}{\partial Y^{2}}+\frac{\partial^{2} \omega}{\partial X^{2}}=K_{o}^{2} e^{-2 \omega}
$$

Following Polyanin and Zaitsev [2004], a solution for (5.9) is given by

$$
\omega(X, Y, t)=-\frac{1}{2} \ln \left(\frac{A^{2}(t)+B^{2}(t)}{K_{o}^{2}(t) \cosh ^{2}(C(t)+A(t) X+B(t) Y)}\right),
$$

for some arbitrary functions of time $A=A(t), B=B(t), C=C(t)$, and $K_{o}(t)$. As an example, and for the purpose of illustrating the non-trivial form the initially flat shell could adopt as a result of a stress-free growth, we consider the following cases:

- We assume that $A(t)=t / \tau, B(t)=t / \tau, C(t)=0$ and $K_{0}(t)=\sqrt{2} t / \tau$. It follows that

$$
\omega(X, Y, t)=\ln \{\cosh [(X+Y) t / \tau]\}, \quad K(X, Y, t)=\frac{\sqrt{2} t / \tau}{\cosh ^{2}[(X+Y) t / \tau]},
$$

such that at $t=0$ they satisfy $\omega(X, Y, 0)=0$ and $K(X, Y, 0)=0$. Therefore, we have the following evolving first and second fundamental forms:

$$
\boldsymbol{G}=\cosh ^{2}[(X+Y) t / \tau]\left(\begin{array}{ll}
1 & 0 \\
0 & 1
\end{array}\right), \quad \boldsymbol{B}=\sqrt{2} t / \tau\left(\begin{array}{cc}
-1 & 0 \\
0 & 1
\end{array}\right) .
$$

- We assume that $A(t)=2 t / \tau, B(t)=0, C(t)=0$ and $K_{0}(t)=2 t / \tau$. It follows that

$$
\omega(X, Y, t)=\ln \{\cosh [2 X t / \tau]\}, \quad K(X, Y, t)=\frac{2 t / \tau}{\cosh ^{2}[2 X t / \tau]},
$$

such that at $t=0$ they satisfy $\omega(X, Y, 0)=0$ and $K(X, Y, 0)=0$. Therefore, we have the following evolving first and second fundamental forms:

$$
\boldsymbol{G}=\cosh ^{2}[2 X t / \tau]\left(\begin{array}{ll}
1 & 0 \\
0 & 1
\end{array}\right), \quad \boldsymbol{B}=2 t / \tau\left(\begin{array}{cc}
-1 & 0 \\
0 & 1
\end{array}\right) .
$$


a)
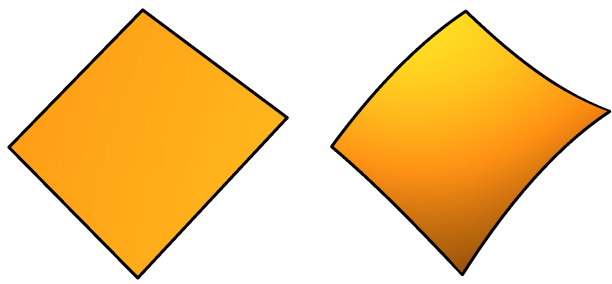

b)
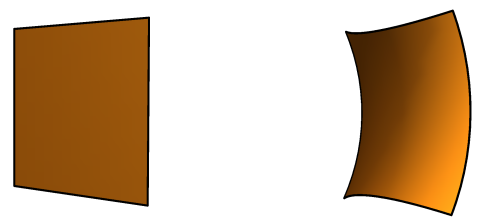

$t=0$
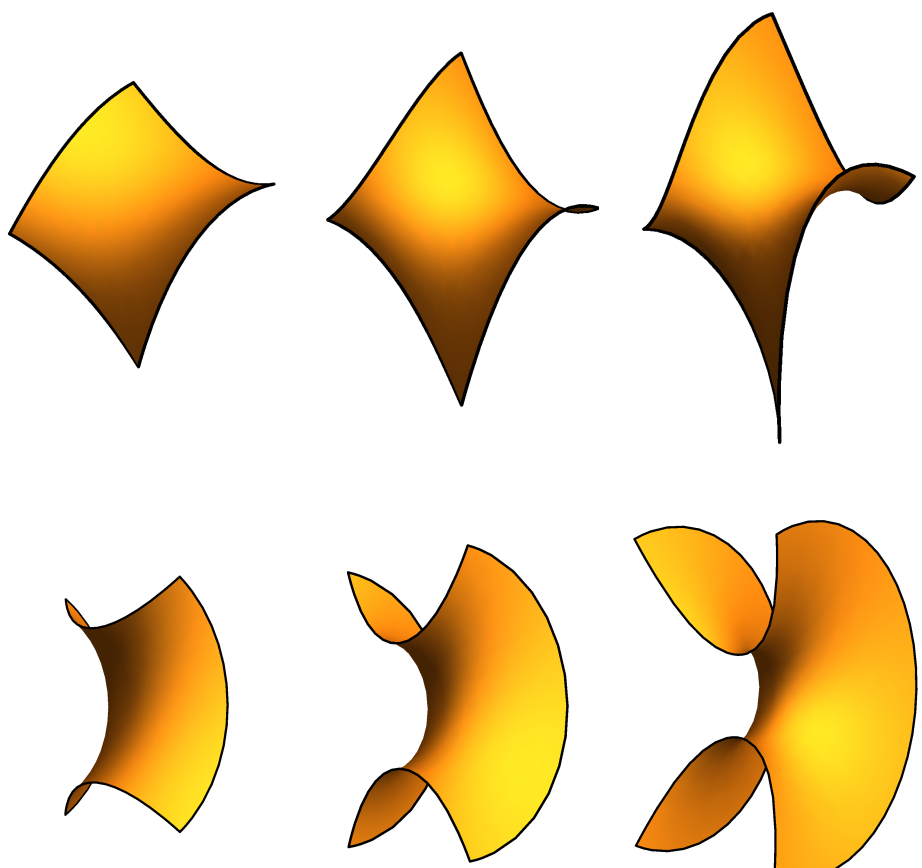

$t=\tau$

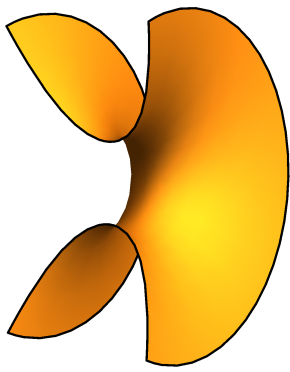

$t=2 \tau$

Figure 8: Example 5.2: Visualization of the stress-free material evolution of an initially planar sheet with the prescribed evolving fundamental forms (5.10) and (5.11), shown, respectively, in a) and b), at different times. Note that the change of shape of the shell is due to growth and not stretch; such an evolution is stress-free.

We visualize in Figure 8, the evolution of the initially planar sheet with the prescribed fundamental forms (5.10) and (5.11).

Remark 5.1. In the previous examples we obtained the first and the second fundamental forms for stress-free growth fields. Recall that a growth field leaves the surface stress-free if and only if it is embeddable in $\mathbb{R}^{3}$. Therefore, given a surface $(\mathcal{H}, \boldsymbol{G}, \boldsymbol{B})$ with a stress-free growth field, we can find an isometric embedding of it in $\mathbb{R}^{3}$ by integrating for the $\mathbb{R}^{3}$-valued function $\boldsymbol{f}$, the following system of partial differential equations written in a local chart $\{X, Y\}$ of $\mathcal{H}$ :

$$
\boldsymbol{f}_{, A B}=\Gamma_{A B}^{C} \boldsymbol{f}_{, C}+\boldsymbol{B}_{A B} \boldsymbol{N},
$$

where $\boldsymbol{N}=\frac{\boldsymbol{f}_{, X} \times \boldsymbol{f}_{, Y}}{\left\|\boldsymbol{f}_{, X} \times \boldsymbol{f}, Y\right\|}, \times$ and $\|\cdot\|$, respectively, denote the cross product and the standard norm in $\mathbb{R}^{3}, \Gamma_{A B}^{C}{ }_{A B}$ is the Christoffel symbol of the Levi-Civita connection $\nabla^{\mathcal{H}}$ in the local chart $\left\{X^{1}, X^{2}\right\}$. The integrability conditions for (5.12) is the equality of the mixed partial for $\boldsymbol{f}$, i.e., $\boldsymbol{f}_{, X Y}=\boldsymbol{f}_{, Y X}$, which is equivalent to the stress-free growth compatibility conditions (or the embeddability conditions) (3.5). Figures 6 , 7 , and 8 are obtained by plotting $\boldsymbol{f}$ in $\mathbb{R}^{3}$ following the numerical integration of (5.12). We fix the rigid body motion of the surface by assuming $\boldsymbol{f}(0,0)=\mathbf{0}, \boldsymbol{f}_{, X}(0,0)=\left(\sqrt{G_{11}(0,0)}, 0,0\right)^{\top}$, and $\boldsymbol{f}_{, Y}(0,0)=\left(0, \sqrt{G_{22}(0,0)}, 0\right)^{\top}$, where T denotes transpose of a vector in $\mathbb{R}^{3}$.

\subsection{An infinitely long morphoelastic circular cylindrical shell}

We consider an infinitely long morphoelastic thin hollow circular cylinder $\mathcal{B}$ under uniform internal pressure $p_{i}=p_{i}(t)$, with thickness $h$ and mid-radius $R_{o}$, made of a homogeneous isotropic material. Let $\mathcal{B}$ undergo a circumferential radially-symmetric but non-uniform growth through its thickness. In the cylindrical coordinates $(R, \Phi, Z)$, such that $R \geq 0,0 \leq \Phi \leq 2 \pi$, and $Z \in \mathbb{R}$, we represent growth by the following evolving material 
metric

$$
\overline{\boldsymbol{G}}=\left(\begin{array}{ccc}
1 & 0 & 0 \\
0 & R^{2} e^{2 \bar{\omega}(R, t)} & 0 \\
0 & 0 & 1
\end{array}\right),
$$

which corresponds to the following evolving first and second fundamental forms for the mid-cylinder $\mathcal{H}$ of radius $R_{o}$ in the coordinate system $(\Phi, Z)$ :

$$
\boldsymbol{G}=\left(\begin{array}{cc}
R_{o}^{2} e^{2 \omega(t)} & 0 \\
0 & 1
\end{array}\right), \quad \boldsymbol{B}=\left(\begin{array}{cc}
-\left[1+R_{0} K(t)\right] R_{0} e^{2 \omega(t)} & 0 \\
0 & 0
\end{array}\right)
$$

where $\omega(t)=\bar{\omega}\left(R_{o}, t\right)$ and $K(t)=\frac{\partial \bar{\omega}}{\partial R}\left(R_{o}, t\right)$.

Let $(r, \phi, z)$ be the cylindrical coordinate system for the Euclidean ambient space. Based on the symmetry of the problem, and in order to find the growth-induced residual stress field, we embed the material manifold into the Euclidean ambient space to form a circular cylindrical shell of radius $r=r(t)$ such that $(\phi, z)=(\Phi, Z)$. The spatial first and second fundamental forms for the cylindrical shell in the cylindrical coordinate system $(\phi, \xi)$ can be written as

$$
\boldsymbol{g}=\left(\begin{array}{cc}
r^{2}(t) & 0 \\
0 & 1
\end{array}\right), \quad \boldsymbol{\beta}=\left(\begin{array}{cc}
-r(t) & 0 \\
0 & 0
\end{array}\right)
$$

Therefore, the deformation tensors read

$$
\boldsymbol{C}=\left(\begin{array}{cc}
r^{2}(t) & 0 \\
0 & 1
\end{array}\right), \quad \boldsymbol{\Theta}=\left(\begin{array}{cc}
-r(t) & 0 \\
0 & 0
\end{array}\right) .
$$

Note that since the cylinder is made of a homogeneous and isotropic material, and because of the radial symmetry of the problem, we have

$$
\begin{array}{lll}
\sigma^{\phi \phi}(t)=\sigma^{\phi \phi}(r(t), \omega(t), K(t)), & \sigma^{z \phi}(t)=0, & \sigma^{z z}(t)=\sigma^{z z}(r(t), \omega(t), K(t)), \\
\mu^{\phi \phi}(t)=\mu^{\phi \phi}(r(t), \omega(t), K(t)), & \mu^{z \phi}(t)=0, & \mu^{z z}(t)=\mu^{z z}(r(t), \omega(t), K(t)) .
\end{array}
$$

It follows that the only non-trivial equilibrium equation is $(4.19 \mathrm{~b})$, which is simplified to read ${ }^{13}$

$$
\left(\sigma^{\phi \phi}-\frac{1}{r} \mu^{\phi \phi}\right) r-p_{i}=0
$$

We assume a Saint Venant-Kirchhoff constitutive model, for which the strain energy density $\mathcal{W}$ is given by (4.31). Therefore, the non-zero components of the material stress and couple stress tensors read

$$
\begin{aligned}
\sigma^{\phi \phi} & =h \frac{E}{2\left(1-\nu^{2}\right)}\left(\frac{r^{2}(t)}{R_{o}^{2}} e^{-2 \omega(t)}-1\right) \frac{1}{r^{2}(t)}, \\
\sigma^{z z} & =h \frac{E \nu}{2\left(1-\nu^{2}\right)}\left(\frac{r^{2}(t)}{R_{o}^{2}} e^{-2 \omega(t)}-1\right) \frac{1}{r^{2}(t)}, \\
\mu^{\phi \phi} & =\frac{h^{3}}{6} \frac{E}{2\left(1-\nu^{2}\right)}\left[\left(1+R_{0} K(t)\right) R_{0} e^{2 \omega(t)}-r(t)\right] \frac{e^{-2 \omega(t)}}{R_{o}^{2} r^{2}(t)}, \\
\mu^{z z} & =\frac{h^{3}}{6} \frac{E \nu}{2\left(1-\nu^{2}\right)}\left[\left(1+R_{0} K(t)\right) R_{0} e^{2 \omega(t)}-r(t)\right] \frac{e^{-2 \omega(t)}}{R_{o}^{2} r^{2}(t)} .
\end{aligned}
$$

\footnotetext{
${ }^{13}$ Following (4.19b), there are three equilibrium equations

$$
\begin{gathered}
\left(\sigma^{a c}+\beta_{b}^{a} \mu^{b c}\right)_{\mid c}+\beta_{b}^{a} \mu^{b c}{ }_{\mid c}=0, \quad \text { for } a=r, \phi \\
\left(\sigma^{a c}+\beta^{a}{ }_{b} \mu^{b c}\right) \beta_{a c}-\mu^{a b}{ }_{\mid a b}+p_{i}=0 .
\end{gathered}
$$

Because of the symmetry of the problem and the isotropy of the material, the stresses take the form (5.13). This implies that equations (5.14a) are trivially satisfied and the terms containing derivatives in (5.14b) vanish. Therefore, we are left with equation (5.15) as the only non-trivial equilibrium equation.
} 
Then, it follows from (5.15) that

$$
\frac{r^{3}(t)}{R_{o}^{3} e^{3 \omega(t)}}-\frac{p_{i}(t) R_{o} e^{\omega(t)}}{Y_{c} h} \frac{r^{2}(t)}{R_{o}^{2} e^{2 \omega(t)}}+\left(\frac{1}{6} \frac{h^{2}}{R_{o}^{2} e^{2 \omega(t)}}-1\right) \frac{r(t)}{R_{o} e^{\omega(t)}}-\frac{1}{6} \frac{h^{2}}{R_{o}^{2} e^{\omega(t)}}\left(1+R_{0} K(t)\right)=0,
$$

where $Y_{c}=\frac{E}{2\left(1-\nu^{2}\right)}=\mu+\frac{\mu \lambda}{2 \mu+\lambda}$. We introduce the following dimensionless quantities:

$$
\hat{h}=\frac{h}{R_{o}}, \quad \hat{K}=R_{o} K, \quad \hat{r}=\frac{r}{R_{o}}, \quad \hat{p}_{i}=\frac{p_{i}}{Y_{c}},
$$

and rewrite (5.17) as

$$
\frac{\hat{r}^{3}(t)}{e^{3 \omega(t)}}-\frac{\hat{p}_{i}(t) e^{\omega(t)}}{\hat{h}} \frac{\hat{r}^{2}(t)}{e^{2 \omega(t)}}+\left(\frac{\hat{h}^{2}}{6 e^{2 \omega(t)}}-1\right) \frac{\hat{r}(t)}{e^{\omega(t)}}-\frac{\hat{h}^{2}}{6 e^{\omega(t)}}(1+\hat{K}(t))=0 .
$$

Equation (5.18) is a cubic equation in $\hat{r}(t) e^{-\omega(t)}$ that has at least one real solution, which is calculated in closed form using the method of Cardano-Tartaglia (See [Cox, 2012] for more details on the method). To obtain closed form solutions for (5.18), we use the following change of variable

$$
\zeta=\frac{\hat{r}(t)}{e^{\omega(t)}}-\frac{\hat{p}_{i}(t) e^{\omega(t)}}{3 h} .
$$

In terms of $\zeta,(5.18)$ reads

$$
\zeta^{3}+m \zeta+n=0
$$

where

$$
m=\frac{\hat{h}^{2}}{6 e^{2 \omega(t)}}-1-\frac{\hat{p}_{i}^{2}(t) e^{2 \omega(t)}}{3 \hat{h}^{2}} \text { and } n=-\frac{\hat{p}_{i}(t) e^{\omega(t)}}{27 \hat{h}}\left(\frac{2 \hat{p}_{i}(t)^{2} e^{2 \omega(t)}}{\hat{h}^{2}}-\frac{3 \hat{h}^{2}}{2 e^{2 \omega(t)}}+9\right)-\frac{\hat{h}^{2}}{6 e^{\omega(t)}}(1+\hat{K}(t)) .
$$

The discriminant of (5.19) reads $\Delta=-4 m^{3}-27 n^{2}$. We distinguish three cases:

i) When $\Delta>0,(5.17)$ has three real solutions, of which we pick the positive one (the physically meaningful one). For $i \in\{0,1,2\}$, these three solutions are

$$
\hat{r}(t)=\frac{\hat{p}_{i}(t) e^{2 \omega(t)}}{3 \hat{h}}+2 e^{\omega(t)} \sqrt{\frac{-m}{3}} \cos \left[\frac{1}{3} \cos ^{-1}\left(\frac{-n}{2} \sqrt{\frac{27}{-m^{3}}}\right)+\frac{2 i \pi}{3}\right] .
$$

ii) When $\Delta=0,(5.17)$ has two real solutions, of which we pick the positive one. These two solutions are

$$
\hat{r}(t)=\frac{\hat{p}_{i}(t) e^{2 \omega(t)}}{3 \hat{h}}+\frac{3 n e^{\omega(t)}}{m}, \quad \hat{r}(t)=\frac{\hat{p}_{i}(t) e^{2 \omega(t)}}{3 \hat{h}}-\frac{3 n e^{\omega(t)}}{2 m} .
$$

iii) When $\Delta<0,(5.17)$ has a unique real solution, which reads

$$
\hat{r}(t)=\frac{\hat{p}_{i}(t) e^{2 \omega(t)}}{3 \hat{h}}+e^{\omega(t)}\left(\frac{-n+\sqrt{\frac{-\Delta}{27}}}{2}\right)^{\frac{1}{3}}+e^{\omega(t)}\left(\frac{-n-\sqrt{\frac{-\Delta}{27}}}{2}\right)^{\frac{1}{3}} .
$$

Now we only need to find the evolution of the first and the second fundamental forms of the material manifold to characterize the growth of the cylindrical shell. We assume the following Rayleigh potential

$$
\mathcal{R}(\dot{\boldsymbol{G}}, \dot{\boldsymbol{B}}, \boldsymbol{G})=\alpha_{1} \operatorname{tr}(\dot{\boldsymbol{G}})+\alpha_{2} \operatorname{tr}\left(\dot{\boldsymbol{G}}^{2}\right)+\beta_{1} \operatorname{tr}(\dot{\boldsymbol{B}})+\beta_{2} \operatorname{tr}\left(\dot{\boldsymbol{B}}^{2}\right) .
$$


Therefore, equations (4.25) yield the following kinetic equations for $\omega$ and $K$

$$
\begin{aligned}
\dot{\omega} & =-\frac{\alpha_{1}}{4 \alpha_{2}}-\frac{Y_{c} h}{32 \alpha_{2}}\left(\frac{r^{2}}{R_{o}^{2}} e^{-2 \omega}-1\right)^{2}+\frac{Y_{c} h}{8 \alpha_{2}}\left(\frac{r^{2}}{R_{o}^{2}} e^{-2 \omega}-1\right) \frac{r^{2}}{R_{o}^{2}} e^{-2 \omega}+\frac{Y_{c} h^{3}}{32 \alpha_{2}}\left(K+\frac{1}{R_{o}}-\frac{r}{R_{o}^{2}} e^{-2 \omega}\right)^{2}, \\
\dot{K} & =-2 \dot{\omega}\left(K+\frac{1}{R_{o}}\right)+\frac{\beta_{1}}{2 \beta_{2}}-\frac{Y_{c} h^{3}}{12 \beta_{2}}\left(K+\frac{1}{R_{o}}-\frac{r}{R_{o}^{2}} e^{-2 \omega}\right) .
\end{aligned}
$$

The system of ordinary differential equations (5.24) along with the initial conditions $\omega(0)=0$ and $K(0)=0$ (see $(2.11)$ ), is an initial-value problem. Following the existence and uniqueness theorems of the initial-value problems [Coddington and Levinson, 1955], $\exists T>0$ such that $(5.24)$ has a unique solution $(\omega, K)$ for $t \in[0, T]$ with $\omega(0)=0$ and $K(0)=0$.

Example 5.3. In this example, we assume that the growth through the thickness of the hollow cylinder $\mathcal{B}$ is nonuniform and symmetric with respect to the mid-cylinder $\mathcal{H}$. In particular, we assume that $K(t)=\frac{\partial \bar{\omega}}{\partial R}\left(R_{o}, t\right) \neq 0$ and $\omega(t)=\bar{\omega}\left(R_{o}, t\right)=0$. Therefore, the kinetic equations reduce to $(5.24 \mathrm{~b}),{ }^{14}$ giving the evolution of $K$, which further simplifies to read ${ }^{15}$

$$
\dot{K}=\frac{\beta_{1}}{2 \beta_{2}}+\frac{Y_{c} h^{3}}{12 \beta_{2}}\left(\frac{r}{R_{o}^{2}}-K-\frac{1}{R_{o}}\right)
$$

We define the following characteristic time

$$
\tau=\frac{12 \beta_{2}}{Y_{c} h^{3}} .
$$

We differentiate with respect to $\hat{t}=t / \tau$ and rewrite (5.25) using dimensionless quantities $\hat{K}$ and $\hat{r}$

$$
\dot{\hat{K}}(\hat{t})=\frac{6 \beta_{1} R_{o}}{Y_{c} h^{3}}+(\hat{r}(\hat{t})-\hat{K}(\hat{t})-1),
$$

where $\hat{r}$ is given by (5.20), (5.21), or (5.22) depending on the sign of the discriminant $\Delta$. Following (5.16), the physical components of the residual stresses read

$$
\begin{aligned}
\hat{\sigma}^{\phi \phi}=h Y_{c}\left(\hat{r}^{2}(t)-1\right), \quad \hat{\sigma}^{z z} & =h Y_{s}\left(\hat{r}^{2}(t)-1\right), \\
\hat{\mu}^{\phi \phi}=h^{2} Y_{c} \frac{\hat{h}}{6}[1+\hat{K}(t)-\hat{r}(t)], & \hat{\mu}^{z z}=h^{2} Y_{s} \frac{\hat{h}}{6}[1+\hat{K}(t)-\hat{r}(t)],
\end{aligned}
$$

where $Y_{s}=\frac{\mu \lambda}{2 \mu+\lambda}$ and recall that $Y_{c}=\mu+\frac{\mu \lambda}{2 \mu+\lambda}$.

In the linear theory of elasticity, it can be shown that the circumferential stress in a thin-walled cylinder of radius $R_{o}$ and thickness $h$, subject to an internal pressure $p_{i}$, is given by

$$
\hat{\sigma}_{\text {lin }}^{\phi \phi}=\frac{p R_{o}}{h} .
$$

Starting from our circumferential shell stress $\hat{\sigma}^{\phi \phi}$, we want to prove that for $p$ and $h$ small, $\hat{\sigma}^{\phi \phi}$ approaches $h \hat{\sigma}_{\text {lin }}^{\phi \phi}$. For $p$ and $h$ small enough, the discriminant $\Delta>0$ and the dimensionless radius is written as

$$
r(p, h, t)=\frac{p_{i} R_{o}^{2}}{3 Y_{c} h}+2 R_{o} \sqrt{\frac{-m}{3}} \cos \left[\frac{1}{3} \cos ^{-1}\left(\frac{-n}{2} \sqrt{\frac{27}{-m^{3}}}\right)\right],
$$

where $m=\frac{h^{2}}{6 R_{o}^{2}}-1-\frac{p_{i}^{2} R_{o}^{2}}{3 Y_{c}^{2} h^{2}}$ and $n=-\frac{p_{i} R_{o}}{27 Y_{c} h}\left(\frac{2 p_{i}^{2} R_{o}^{2}}{Y_{c}^{2} h^{2}}-\frac{3 h^{2}}{2 R_{o}^{2}}+9\right)-\frac{h^{2}}{6 R_{o}^{2}}\left(1+R_{o} K(t)\right)$. For small $p$ and $h$, we have the following asymptotic expansion

$$
r(p, h, t)=R_{o}+\left[\frac{R_{o}^{2}}{2 h}+o(h)\right] \frac{p}{Y_{c}}+o(h)+o\left(p^{2}\right) .
$$

\footnotetext{
${ }^{14}$ When $\omega=0$, the first fundamental form $\boldsymbol{G}$ is not a dynamical variable anymore, and hence, the kinetic equation (5.24a) should be discarded.

${ }^{15}$ Recall that $r$ depends on $K$ as can be seen in (5.20), (5.21), or (5.22) depending on the value of the discriminant $\Delta$.
} 


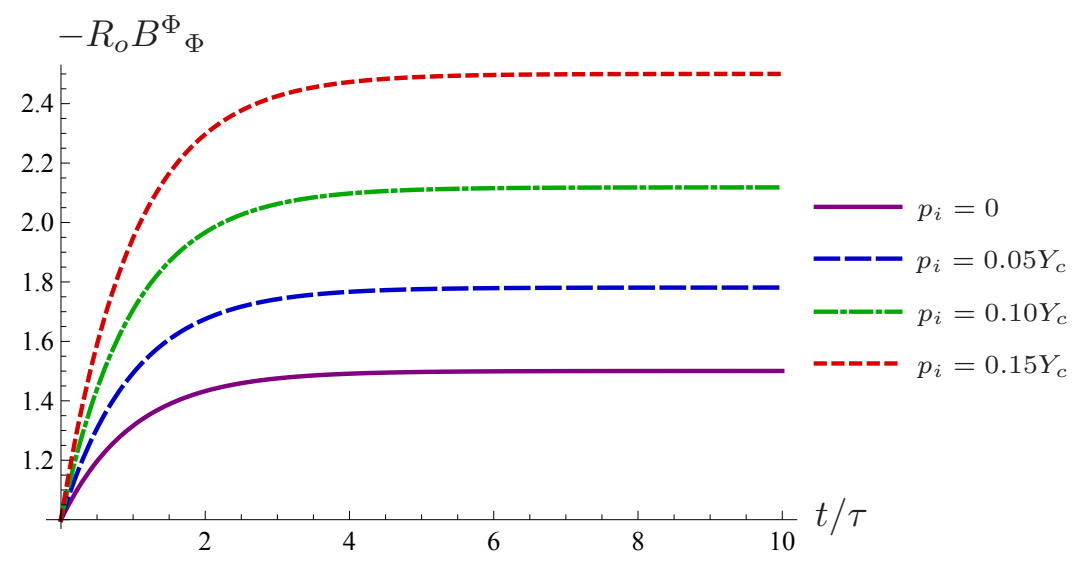

Figure 9: The evolution of the material curvature $B_{\Phi}^{\Phi}$ of a morphoelastic circular cylindrical shell with $h=0.1 R_{o}$ for different values of the constant internal pressure $p_{i}$.

It follows that

$$
\frac{\hat{\sigma}^{\phi \phi}}{h}=Y_{c}\left(\frac{r^{2}(t)}{R_{o}^{2}}-1\right)=\frac{p R_{o}}{h}+o(h) p+o\left(h^{2}\right)+o\left(p^{2}\right) .
$$

For a shell of thickness $h=0.1 R_{o}$, a growth such that $\beta_{1}=Y_{c} h^{3} /\left(12 R_{o}\right)$, and for different values of a constant internal pressure $p_{i}$, we numerically solve (5.27) for the evolution of $K$ (see Figure 9 for the evolution of the material curvature $B_{\Phi}^{\Phi}=-\frac{1}{R_{o}}-K(t)$ ). We consequently obtain the evolution of its radius and the corresponding circumferential stress and couple stress (see Figures 10, 11, and 12). We also consider a timedependent internal pressure and numerically solve (5.27) for the evolution of $K$. We assume in particular a time-dependent profile of internal pressure given in Figure 13. We show, in Figure 14 the evolution of the material curvature $B^{\Phi}$ and the resulting couple stress induced by the time-dependent internal pressure shown in Figure 13.

We note that the cylindrical shell expands and contracts following the internal pressure level, through the explicit dependence of the spatial radius $r$ on the internal pressure $p_{i}$. Also, the circumferential stress follows the profile of expansion and contraction of the cylinder and has a value close to that of the thin walled cylinders, i.e., $\frac{p_{i} r}{h}$. Note that we have omitted the plots of evolution of the radius and the circumferential stress as their profiles are similar to that of the internal pressure. We note that $\hat{\mu}^{\phi \phi}=\beta_{1}-h^{2} Y_{c} \frac{\hat{h}}{6} \dot{\hat{K}}(\hat{t})=\beta_{1}+h^{2} Y_{c} \frac{\hat{h}}{6} \dot{B}^{\Phi}{ }_{\Phi}(\hat{t})$, and we can hence interpret, in this case, the circumferential couple stress as the rate of change of the material curvature augmented by the growth-induced residual couple stress $\beta_{1}$. We observe that at large times, the rate of change of the curvature $\dot{\hat{K}}$ tends to zero (suggesting that the system (5.27) asymptotically tends to its attracting fixed point). Therefore, in the case of the constant internal pressure, the circumferential stress tends to the residual couple-stress $\beta_{1}$, and in the case of a time-dependent pressure, the mean value of the circumferential stress tends to the residual couple-stress $\beta_{1}$. Note that the plots of the longitudinal stress and couple stress are omitted because they are, respectively, proportional to the circumferential stress and couple stress by a factor of $Y_{s} / Y_{c}=\nu$.

\subsection{A morphoelastic circular shell}

In the absence of body forces, we consider an initially planar thin morphoelastic circular disk $\mathcal{B}$ with vanishing boundary loads. We assume that the disk is undergoing radially-symmetric but non-uniform growth through its thickness such that the radial and circumferential curvatures are evolving while the intrinsic metric of the shell remains unchanged. Let $(R, \Phi, Z)$ be the standard cylindrical coordinate system for $\mathbb{R}^{3}$ such that initially the mid-surface $\mathcal{H}$ of the shell lies in the hyperplane $Z=0$ and the origin of the coordinate system coincides with the center of the circular mid-surface. Let $R_{o}$ be the radius of the circular disk. For time $t \geq 0$, we represent 


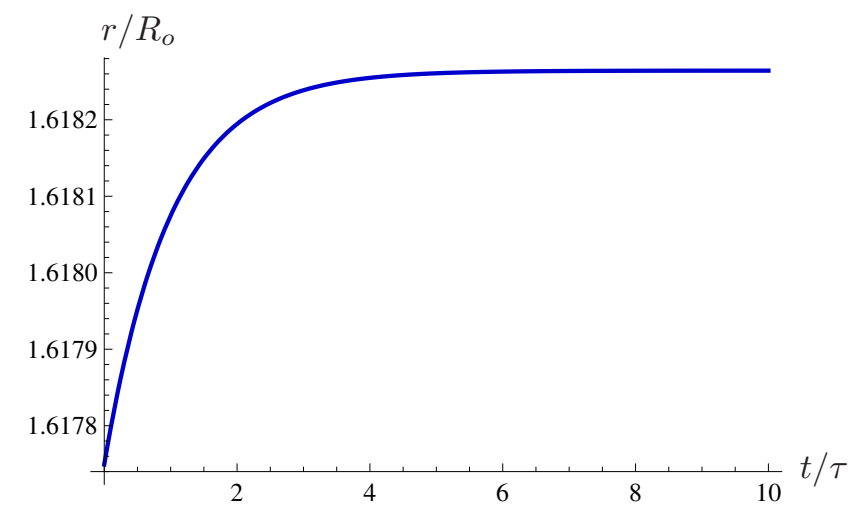

(i)

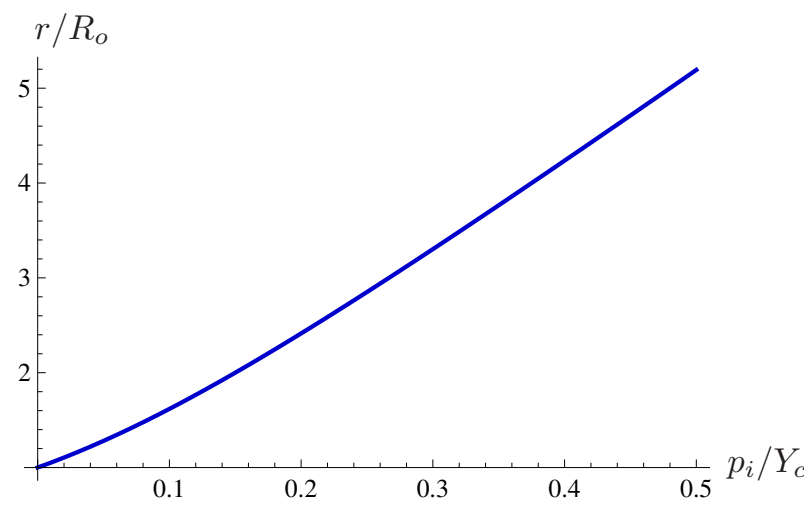

(ii)

Figure 10: The evolution of the radius $r$ of a morphoelastic circular cylindrical shell with $h=0.1 R_{o}$. (i) shows the time-evolution for a constant internal pressure $p_{i}=0.1 Y_{c}$, and (ii) shows the internal pressure dependence at time $t=10 \tau$.

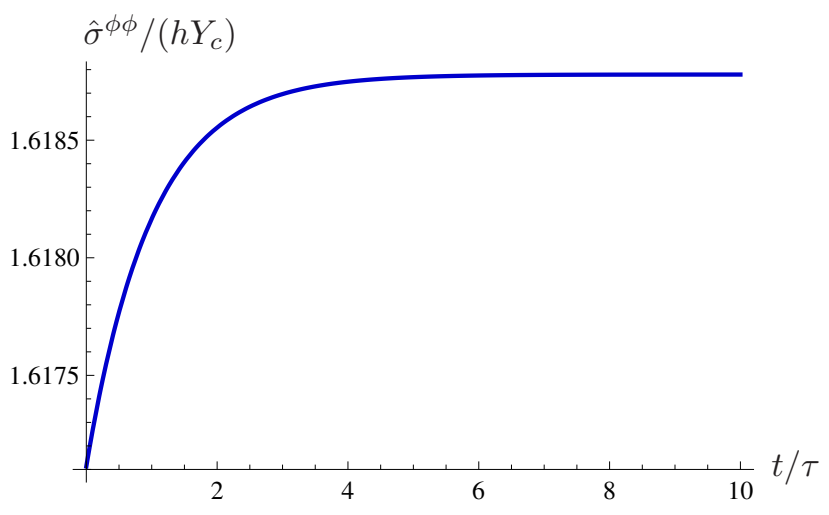

(i)

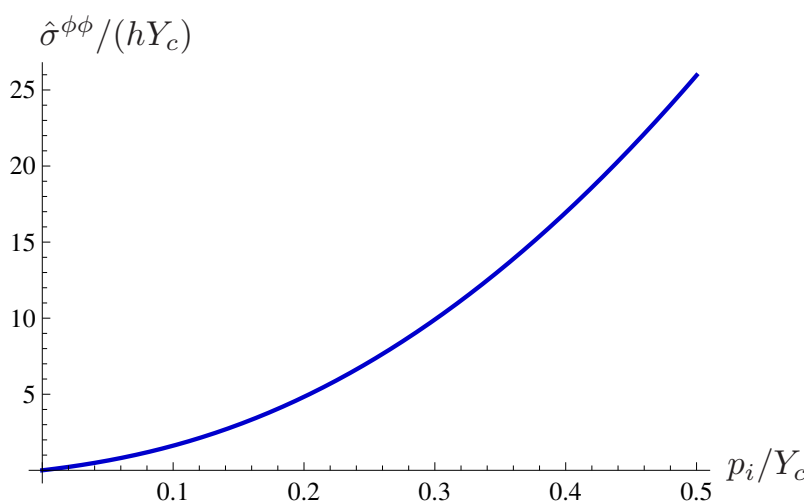

(ii)

Figure 11: The evolution of the circumferential stress of a morphoelastic circular cylindrical shell with $h=0.1 R_{o}$. (i) shows the time-evolution for a constant internal pressure $p_{i}=0.1 Y_{c}$, and (ii) shows the internal pressure dependence at time $t=10 \tau$.

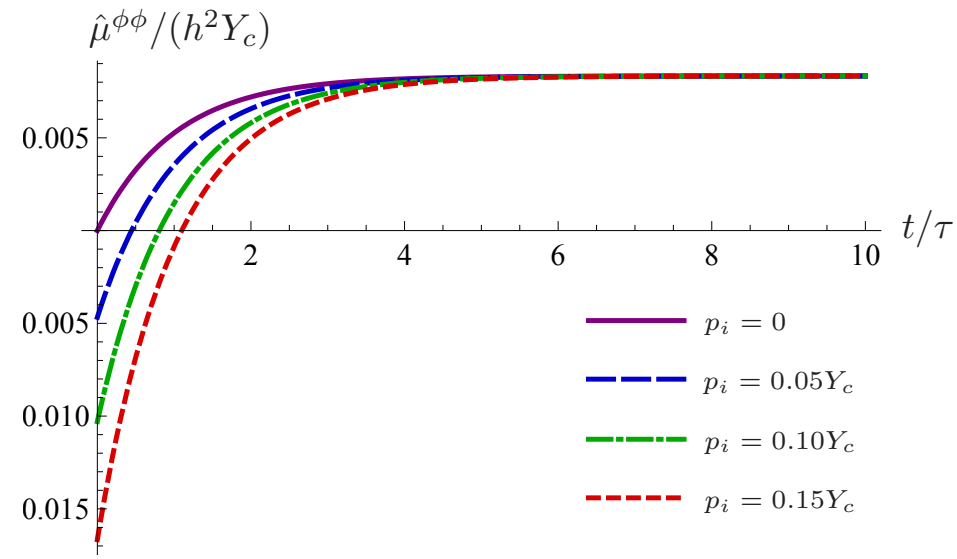

Figure 12: The evolution of the circumferential couple stress of a morphoelastic circular cylindrical shell with $h=0.1 R_{o}$ for different values of the constant internal pressure $p_{i}$. 


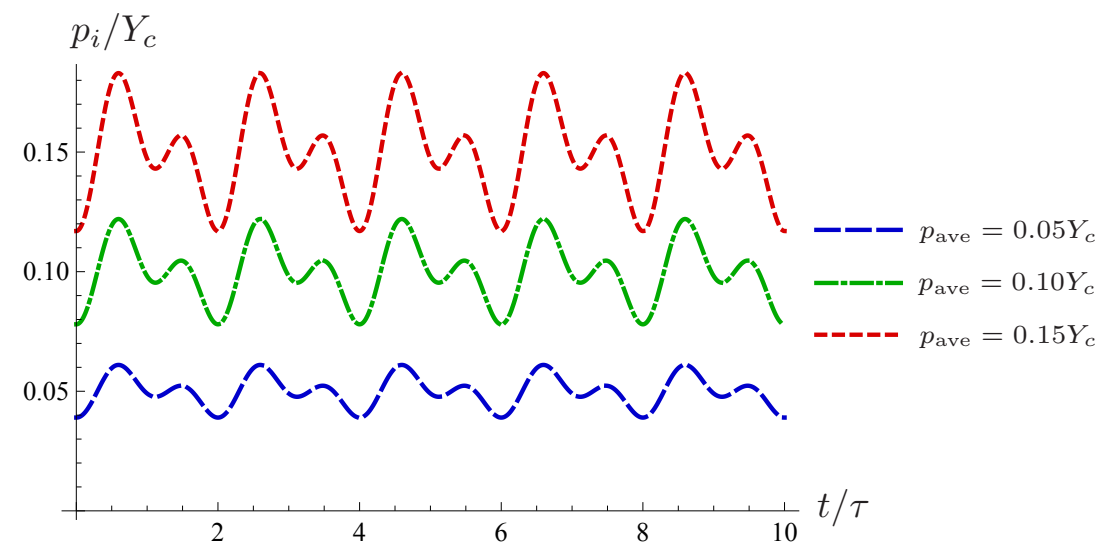

Figure 13: The time-dependent profiles for internal pressure $p_{i}$. Each profile corresponds to its average pressure value $p_{\text {ave }}$.

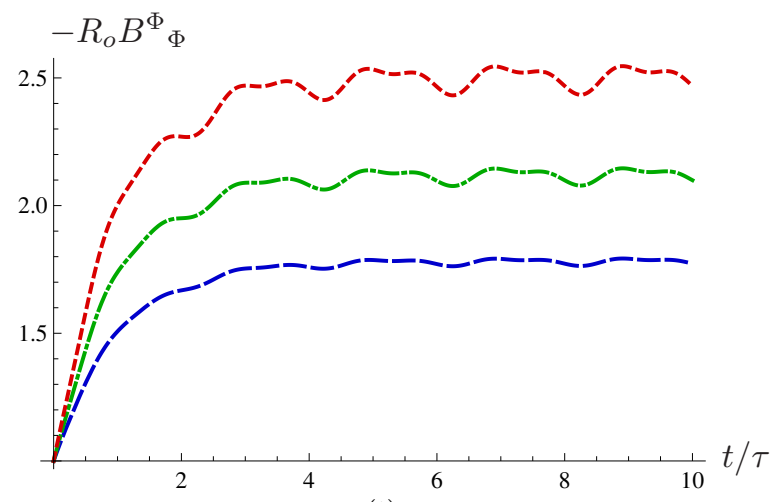

(i)

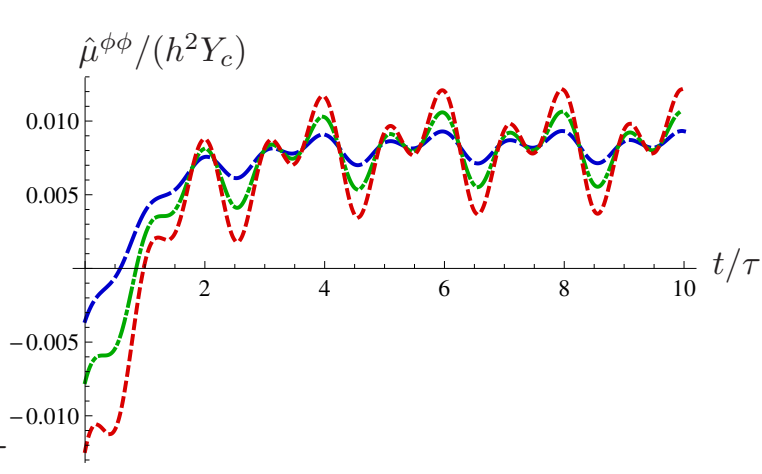

(ii)

$---p_{\text {ave }}=0.05 Y_{c} \quad----p_{\text {ave }}=0.10 Y_{c} \quad-----p_{\text {ave }}=0.15 Y_{c}$

Figure 14: The evolution of (i) the material curvature $B_{\Phi}^{\Phi_{\Phi}}$, and (ii) the circumferential couple stress of a morphoelastic circular cylindrical shell with $h=0.1 R_{o}$ for the time-dependent internal pressure plotted in Figure 13. 
the aforementioned growth by the following evolving material metric:

$$
\overline{\boldsymbol{G}}=\left(\begin{array}{ccc}
e^{2 \omega_{R}(R, Z, t)} & 0 & 0 \\
0 & e^{2 \omega_{\Theta}(R, Z, t)} R^{2} & 0 \\
0 & 0 & 1
\end{array}\right)
$$

such that $\omega_{A}$ for $A=R, \Theta$ are symmetric with respect to $Z$, i.e., $\omega_{A}(R, Z, t)=-\omega_{A}(R,-Z, t)$, which implies that $\omega_{A}(R, 0, t)=0 .{ }^{16}$ Therefore, the first and the second fundamental forms of $\mathcal{H}$ read

$$
\boldsymbol{G}=\left(\begin{array}{cc}
1 & 0 \\
0 & R^{2}
\end{array}\right), \quad \boldsymbol{B}=\left(\begin{array}{cc}
-K_{R}(R, t) & 0 \\
0 & -R^{2} K_{\Theta}(R, t)
\end{array}\right),
$$

where $K_{A}(R, t)=\frac{\partial \omega_{A}}{\partial Z}(R, 0, t)$ for $A=R, \Theta$.

We endow the ambient Euclidean space $\mathbb{R}^{3}$ with the standard cylindrical coordinate system $(r, \phi, z)$. In order to study the growth of the shell and obtain the growth-induced residual stresses, we embed the shell into the Euclidean ambient space and look for solutions of the form $(r, \phi, z)=(r(R, t), \Phi, z(R, t))$. We fix the rigid body motion of the embedded surface by assuming $r(0, t)=0, z(0, t)=0$, and $z^{\prime}(0, t)=0$. Therefore, the deformation tensors read

$$
\boldsymbol{C}=\left(\begin{array}{cc}
{r^{\prime}}^{2}+z^{\prime 2} & 0 \\
0 & r^{2}
\end{array}\right), \quad \boldsymbol{\Theta}=\frac{1}{\left({r^{\prime}}^{2}+z^{\prime 2}\right)^{1 / 2}}\left(\begin{array}{cc}
r^{\prime} z^{\prime \prime}-r^{\prime \prime} z^{\prime} & 0 \\
0 & r z^{\prime}
\end{array}\right)
$$

where $r^{\prime}=\frac{\partial r}{\partial R}$ and $r^{\prime \prime}=\frac{\partial^{2} r}{\partial R^{2}}$. We introduce the function $\chi=\chi(R, t)$ defined such that $z^{\prime}=\chi r^{\prime}$. Hence, the deformation tensors in terms of $r$ and $\chi$ read

$$
\boldsymbol{C}=\left(\begin{array}{cc}
{r^{\prime}}^{2}\left(1+\chi^{2}\right) & 0 \\
0 & r^{2}
\end{array}\right), \quad \boldsymbol{\Theta}=\frac{1}{\left(1+\chi^{2}\right)^{1 / 2}}\left(\begin{array}{cc}
\chi^{\prime} r^{\prime} & 0 \\
0 & \chi r
\end{array}\right)
$$

We assume that the shell is made of a homogeneous and isotropic material. Because of the symmetry of the problem, the second Piola-Kirchhoff stress and material couple stress components have the following forms

$$
\begin{array}{ccc}
S^{R R}=S^{R R}(R, t), & S^{R \Phi}=0, & S^{\Phi \Phi}=S^{\Phi \Phi}(R, t), \\
M^{R R}=M^{R R}(R, t), & M^{R \Phi}=0, & M^{\Phi \Phi}=M^{\Phi \Phi}(R, t) .
\end{array}
$$

In the convected manifold $(\mathcal{H}, \boldsymbol{C})$, the only non-zero Christoffel symbols of the Levi-Civita connection are

$$
\check{\Gamma}_{R R}^{R}=\frac{\chi \chi^{\prime}}{1+\chi^{2}}+\frac{r^{\prime \prime}}{r^{\prime}}, \quad \check{\Gamma}_{\Phi \Phi}^{R}=-\frac{r}{r^{\prime}\left(1+\chi^{2}\right)}, \quad \check{\Gamma}_{R \Phi}^{\Phi}=\frac{r^{\prime}}{r} .
$$

\footnotetext{
${ }^{16}$ We let for example $\omega_{A}=Z K_{A}(R, t)$ for $A=R, \Theta$ in (5.29).
} 
Therefore, the equilibrium equations follow from (4.21) and read ${ }^{17}$

$$
\begin{array}{r}
\frac{1}{R}\left[R\left(S^{R R}+2 \frac{\chi^{\prime}}{r^{\prime}\left(1+\chi^{2}\right)^{3 / 2}} M^{R R}\right)\right]_{, R}+2\left(\frac{\chi \chi^{\prime}}{1+\chi^{2}}+\frac{r^{\prime \prime}}{r^{\prime}}\right)\left(S^{R R}+2 \frac{\chi^{\prime}}{r^{\prime}\left(1+\chi^{2}\right)^{3 / 2}} M^{R R}\right) \\
-\frac{r}{r^{\prime}\left(1+\chi^{2}\right)}\left(S^{\Phi \Phi}+2 \frac{\chi}{r\left(1+\chi^{2}\right)^{1 / 2}} M^{\Phi \Phi}\right)-\left(\frac{\chi^{\prime}}{r^{\prime}\left(1+\chi^{2}\right)^{3 / 2}}\right)_{, R} M^{R R} \\
-\left(\frac{\chi^{\prime}}{r^{\prime}\left(1+\chi^{2}\right)^{3 / 2}}-\frac{\chi}{r\left(1+\chi^{2}\right)^{1 / 2}}\right) \frac{r}{r^{\prime}\left(1+\chi^{2}\right)} M^{\Phi \Phi}=0 \\
-\frac{\chi^{\prime} r^{\prime}}{\left(1+\chi^{2}\right)^{1 / 2}}\left(S^{R R}+\frac{\chi^{\prime}}{r^{\prime}\left(1+\chi^{2}\right)^{3 / 2}} M^{R R}\right)+\frac{\chi^{r}}{\left(1+\chi^{2}\right)^{1 / 2}}\left(S^{\Phi \Phi}+\frac{\chi}{r\left(1+\chi^{2}\right)^{1 / 2}} M^{\Phi \Phi}\right) \\
\left.-\frac{\chi \chi^{\prime}}{1+\chi^{2}}+\frac{r^{\prime \prime}}{r^{\prime}}\right)\left[\frac{1}{r}\left(R M^{R R}\right)_{, R}+2 \frac{R}{r}\left(\frac{\chi \chi^{\prime}}{1+\chi^{2}}+\frac{r^{\prime \prime}}{r^{\prime}}\right) M^{R R}-\frac{R}{r^{\prime}\left(1+\chi^{2}\right)} M^{\Phi \Phi}\right] \\
\left.{ }_{, R}+2 \frac{R}{r}\left(\frac{\chi \chi^{\prime}}{1+\chi^{2}}+\frac{r^{\prime \prime}}{r^{\prime}}\right) M^{R R}-\frac{R}{r^{\prime}\left(1+\chi^{2}\right)} M^{\Phi \Phi}\right]_{, R}=0 .
\end{array}
$$

The boundary conditions (4.22) for zero surface load, zero moment load, and zero shear load at $R=R_{o}$ read

$$
S^{R R}=0, \quad M^{R R}=0, \quad \frac{1}{R}\left(R M^{R R}\right)^{\prime}-r r^{\prime} M^{\Phi \Phi}=0 .
$$

We assume a Saint Venant-Kirchhoff constitutive model, for which the strain energy density $\mathcal{W}$ is given by (4.31). Therefore, the non-zero components of the material stress and couple stress tensors read

$$
\begin{aligned}
S^{R R} & =Y_{c} h\left\{\left[r^{\prime 2}\left(1+\chi^{2}\right)-1\right]+\nu\left(\frac{r^{2}}{R^{2}}-1\right)\right\}, \\
S^{\Phi \Phi} & =Y_{c} h\left\{\nu\left[r^{\prime 2}\left(1+\chi^{2}\right)-1\right]+\left(\frac{r^{2}}{R^{2}}-1\right)\right\} \frac{1}{R^{2}}, \\
M^{R R} & =Y_{c} h^{3}\left\{\left[\frac{\chi^{\prime} r^{\prime}}{\left(1+\chi^{2}\right)^{1 / 2}}+K_{R}\right]+\nu\left[\frac{\chi^{r}}{R^{2}\left(1+\chi^{2}\right)^{1 / 2}}+K_{\Theta}\right]\right\}, \\
M^{\Phi \Phi} & =Y_{c} h^{3}\left\{\nu\left[\frac{\chi^{\prime} r^{\prime}}{\left(1+\chi^{2}\right)^{1 / 2}}+K_{R}\right]+\left[\frac{\chi r}{R^{2}\left(1+\chi^{2}\right)^{1 / 2}}+K_{\Theta}\right]\right\} \frac{1}{R^{2}},
\end{aligned}
$$

where $Y_{c}=\frac{E}{2\left(1-\nu^{2}\right)}$. Therefore, we have the governing equations (5.31) and the boundary conditions (5.32) in terms of $r$ and $\chi$. However, we still need the kinetic equations for the evolution of growth in order to fully solve the problem. We consider the Rayleigh potential $\mathcal{R}(\dot{\boldsymbol{B}}, \boldsymbol{G})=\beta_{1} \operatorname{tr}(\dot{\boldsymbol{B}})+\beta_{2} \operatorname{tr}\left(\dot{\boldsymbol{B}}^{2}\right)$. Therefore, we find the following evolution equations for the principal curvatures:

$$
\begin{aligned}
& \dot{K}_{R}=\frac{\beta_{1}}{2 \beta_{2}}-\frac{Y_{c} h^{3}}{12 \beta_{2}}\left\{\left[\frac{\chi^{\prime} r^{\prime}}{\left(1+\chi^{2}\right)^{1 / 2}}+K_{R}\right]+\nu\left[\frac{\chi r}{R^{2}\left(1+\chi^{2}\right)^{1 / 2}}+K_{\Theta}\right]\right\}, \\
& \dot{K}_{\Theta}=\frac{\beta_{1}}{2 \beta_{2}}-\frac{Y_{c} h^{3}}{12 \beta_{2}}\left\{\nu\left[\frac{\chi^{\prime} r^{\prime}}{\left(1+\chi^{2}\right)^{1 / 2}}+K_{R}\right]+\left[\frac{\chi r}{R^{2}\left(1+\chi^{2}\right)^{1 / 2}}+K_{\Theta}\right]\right\} .
\end{aligned}
$$

Using the time differentiation with respect to $\hat{t}=t / \tau$ where $\tau$ is defined by (5.26) and the spatial differentiation

\footnotetext{
${ }^{17}$ Note that $\left(\Sigma^{A B}+C^{-A C} \Theta_{C D} \Lambda^{D B}\right)_{\| B}+C^{-A C} \Theta_{C D} \Lambda^{D B} \| B_{\| B}=\left(\Sigma^{A B}+2 C^{-A C} \Theta_{C D} \Lambda^{D B}\right)_{\| B}-\left(C^{-A C} \Theta_{C D}\right)_{\| B} \Lambda^{D B}$. Also, we have $\mathrm{J}=\frac{r}{R}$. Therefore, the convected stress and couple stress tensors read $\boldsymbol{\Sigma}=\frac{R}{r} \boldsymbol{S}$ and $\boldsymbol{\Lambda}=\frac{R}{r} \boldsymbol{M}$.
} 


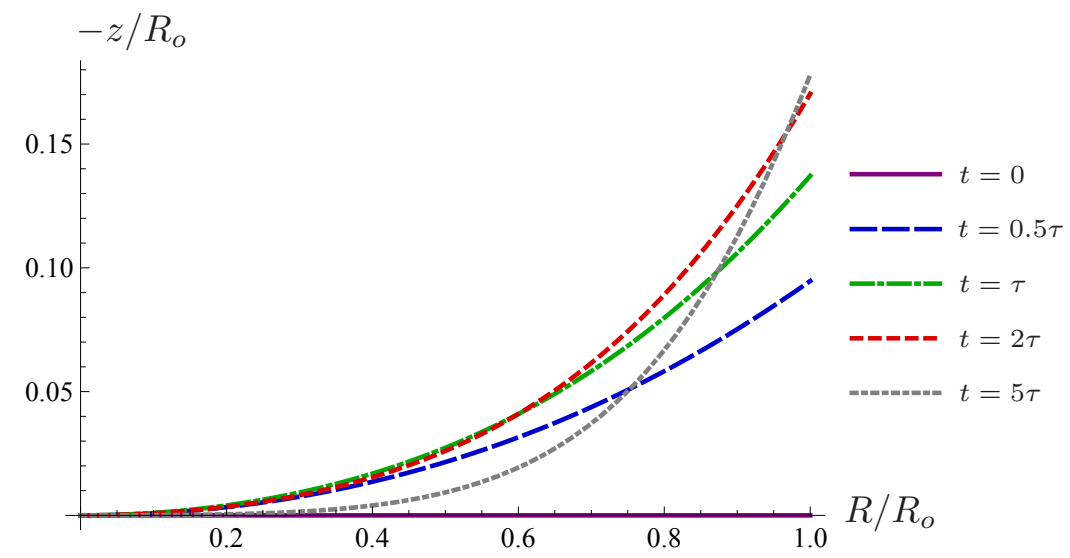

Figure 15: Evolution of (i) the spatial radius $r=r(R, t)$ and (ii) the out-of-plane spatial elevation profile $z=z(R, t)$ of a radial fiber of the initially planar growing disk.

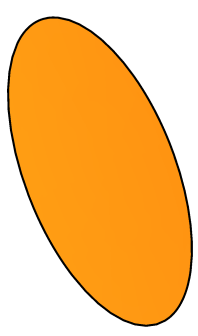

$t=0$

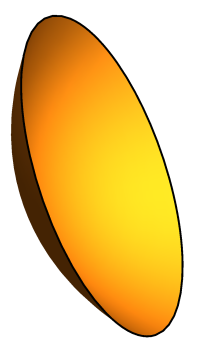

$t=0.5 \tau$

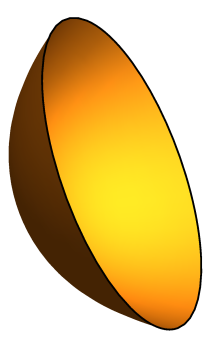

$t=\tau$

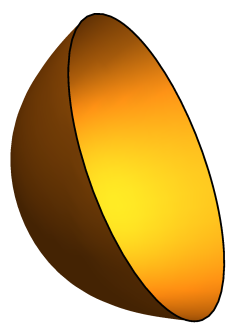

$t=2 \tau$

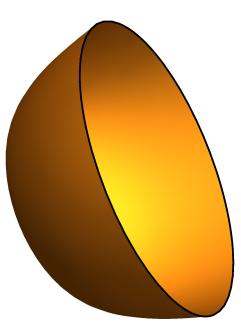

$t=5 \tau$

Figure 16: Visualization of the evolution of an initially planar growing disk. The out-of-plane elevation is scaled to a factor of 5.

with respect to $\hat{R}=R / R_{o},(5.34)$ reads

$$
\begin{aligned}
& \dot{\hat{K}}_{R}=\frac{6 \beta_{1} R_{o}}{Y_{c} h^{3}}-\left\{\left[\frac{\chi^{\prime} \hat{r}^{\prime}}{\left(1+\chi^{2}\right)^{1 / 2}}+\hat{K}_{R}\right]+\nu\left[\frac{\chi \hat{r}}{\hat{R}^{2}\left(1+\chi^{2}\right)^{1 / 2}}+\hat{K}_{\Theta}\right]\right\}, \\
& \dot{\hat{K}}_{\Theta}=\frac{6 \beta_{1} R_{o}}{Y_{c} h^{3}}-\left\{\nu\left[\frac{\chi^{\prime} \hat{r}^{\prime}}{\left(1+\chi^{2}\right)^{1 / 2}}+\hat{K}_{R}\right]+\left[\frac{\chi \hat{r}}{\hat{R}^{2}\left(1+\chi^{2}\right)^{1 / 2}}+\hat{K}_{\Theta}\right]\right\},
\end{aligned}
$$

where $\hat{K}_{A}=K_{A} R_{o}$ for $A=R, \Theta, \hat{r}=\frac{r}{R_{o}}, \hat{R}=\frac{R}{R_{o}}$, and $\hat{h}=\frac{h}{R_{o}}$.

We numerically solve the governing equations (5.31) and the boundary conditions (5.32) along with the kinetic equations (5.34) for a shell of thickness $h=0.1 R_{o}$ made of an isotropic and homogeneous material with $\nu=0.5$, and undergoing a growth such that $\beta_{1}=Y_{c} h^{3} /\left(12 R_{o}\right)$. In Figure 15 we show the evolution of a radial fiber by plotting its radius $r$ and elevation $z$ as a function of $R$. We observe that the radius $r$ remains almost unchanged from $R$ and is almost time-independent while the out-of-plane elevation changes the fiber from its original configuration on the plane $z=0$ to adopt a curved configuration. In Figure 16, we show the evolution of the spatial embedding of the disk from its initial planar configuration to a non-trivial curved disk. In Figure 17, we show the evolution of the radial and the circumferential curvatures. In Figure 18, we show the evolutions of the residual stresses and couple-stresses in the growing disk.

\section{Concluding Remarks}

In this paper, we presented within the framework of nonlinear elasticity, a geometric theory of morphoelastic shells to study the coupling of bulk growth with elasticity. We looked at how bulk growth affects the geometry 


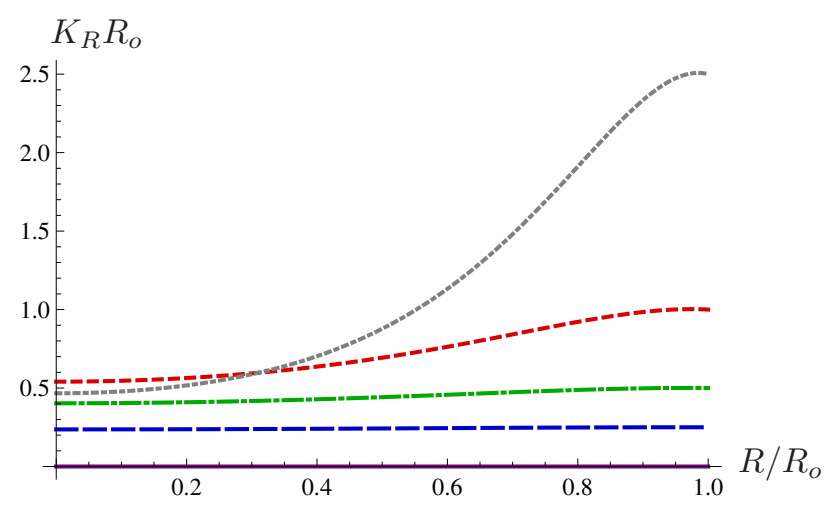

(i)

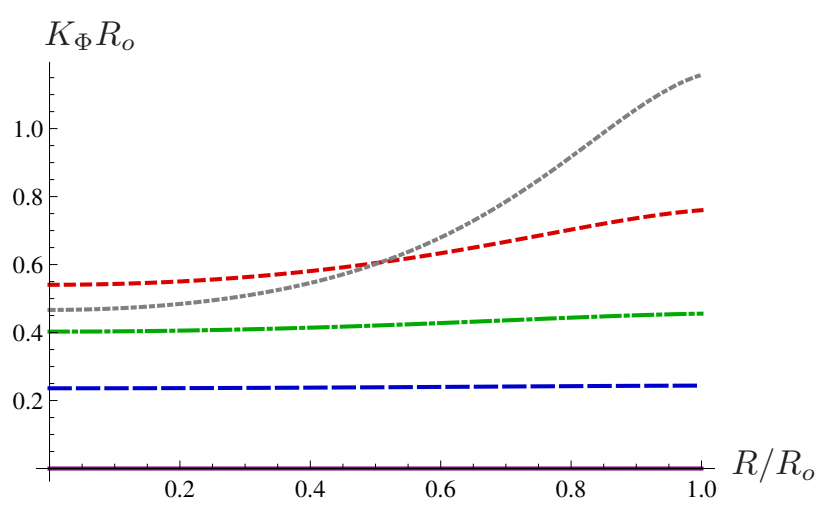

(ii)

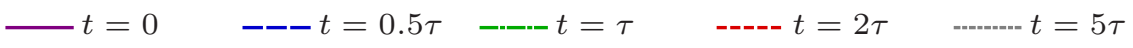

Figure 17: Evolution of the radial (i) and circumferential (ii) principal curvatures on a radial fiber of the initially planar growing disk.

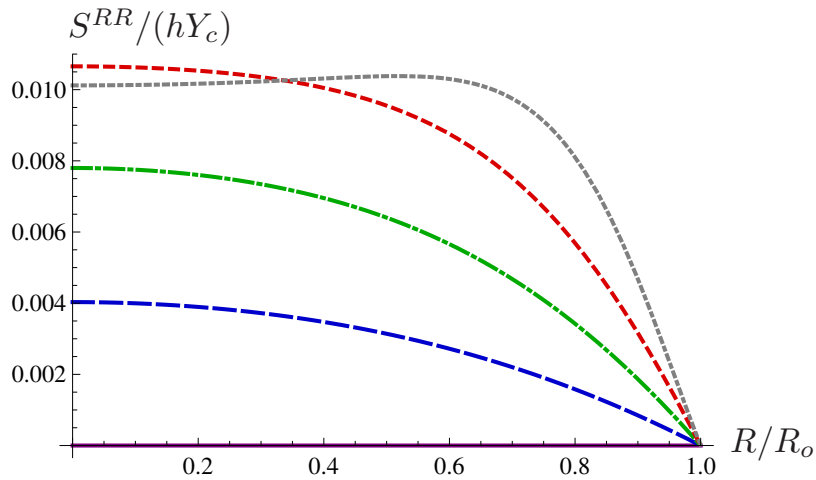

(i)

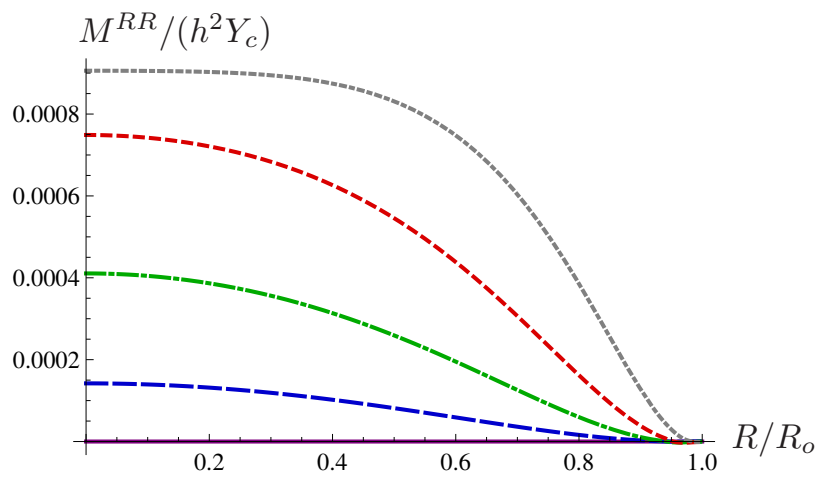

(ii)

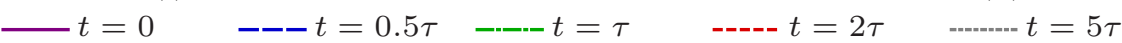

Figure 18: Evolution of (i) the radial stress and (ii) the radial couple-stresses on a radial fiber of the initially planar growing disk. 
of the shell and eventually leads to residual stresses and couple-stresses in the shell. We identified the thin body with a three-dimensional Riemannian manifold and modeled the shell by the mid-surface of the thin body as an orientable two-dimensional Riemannian submanifold. The shell geometry is hence characterized by its first and second fundamental forms that, respectively, represent the in-plane and out-of plane geometries. The nonlinear shell compatibility equations were related to the Gauss and Codazzi-Mainardi equations. We modeled the growth of a shell by considering a referential evolution such that the material points remain fixed while the fundamental forms evolve to account for growth. The evolution of the fundamental forms is such that the shell always remains stress-free in the material manifold, which is not necessarily Euclidean. We discussed a systematic method to obtain those growth fields that leave a simply-connected shell both stress-free and couple-stress-free. Using a Lagrangian field theory, we derived the governing equations of motion. We obtained the balance laws for morphoelatic shells and a kinetic equation governing the evolution of the growth through imposing a coupling between the state of stress of the shell and the time-evolution of the fundamental forms. As an example, we considered a planar sheet and found a family of stress-free growth fields. We observed that stress-free growth can evolve a planar shell into another flat shell, a positively curved, or a negatively curved one. We studied the growth of a morphoelastic infinitely long circular cylindrical shell subject to a time-dependent internal pressure. We also considered a growing circular shell that evolves to a curved cap. In both cases, we numerically obtained the evolution of the evolving curvatures and the induced residual stresses and couple-stresses.

Acknowledgments. SS was supported by a Fulbright Grant. AG is a Wolfson/Royal Society Merit Award Holder and acknowledges support from a Reintegration Grant under EC Framework VII. We thank M.F. Shojaei for his help with some of the numerical examples. This research was partially supported by AFOSR - Grant No. FA9550-12-1-0290 and NSF - Grant No. CMMI 1042559 and CMMI 1130856.

\section{References}

D. Ambrosi and F. Guana. Stress-modulated growth. Mathematics and mechanics of solids, 12(3):319-342, 2007.

D. Ambrosi, G. Ateshian, E. Arruda, S. Cowin, J. Dumais, A. Goriely, G. Holzapfel, J. Humphrey, R. Kemkemer, E. Kuhl, J. Olberding, L. Taber, and K. Garikipati. Perspectives on biological growth and remodeling. Journal of the Mechanics and Physics of Solids, 59(4):863-883, 2011.

A. Angoshtari and A. Yavari. Differential complexes in continuum mechanics. Archive for Rational Mechanics and Analysis, 216(1):193-220, 2015.

H. Aron. Das gleichgewicht und die bewegung einer unendlich dünnen, beliebig gekrümmten elastischen schale. Journal für die reine und angewandte Mathematik, 78:136-174, 1874.

M. Ben Amar and A. Goriely. Growth and instability in elastic tissues. Journal of the Mechanics and Physics of Solids, 53(10):2284-2319, 2005.

O. Bonnet. Mémoire sur la théorie des surfaces applicables sur une surface donnée. Journal de l'École Polytechnique, 24:209 - 230, 1865.

W.-Z. Chien. The intrinsic theory of thin shells and plates i. Quarterly of Applied Mathematics, $1,1943$.

E. F. F. Chladni. Die Akustik. Breitkopf \& Härtel, 1830.

C. Chuong and Y. Fung. Residual stress in arteries. In Frontiers in Biomechanics, pages 117-129. Springer, 1986.

A. Coddington and N. Levinson. Theory of Ordinary Differential Equations. International series in pure and applied mathematics. Tata McGraw-Hill, 1955. ISBN 9780070992566.

E. Cosserat and F. Cosserat. Théories des Corps Déformables. Hermann, Paris, 1909. 
D. Cox. Galois Theory. Pure and Applied Mathematics: A Wiley Series of Texts, Monographs and Tracts. John Wiley \& Sons, 2012. ISBN 9781118218426.

P. P. Delsanto, C. Guiot, P. G. Degiorgis, C. A. Condat, Y. Mansury, and T. S. Deisboeck. Growth model for multicellular tumor spheroids. Applied physics letters, 85(18):4225-4227, 2004.

J. Dervaux, P. Ciarletta, and M. Ben Amar. Morphogenesis of thin hyperelastic plates: a constitutive theory of biological growth in the föppl-von kármán limit. Journal of the Mechanics and Physics of Solids, 57(3): 458-471, 2009.

M. do Carmo. Differential Geometry of Curves and Surfaces. Prentice-Hall, New Jersey, 1976.

M. do Carmo. Riemannian Geometry [translated by F. Flahetry from the 1988 Portuguese edition]. Mathematics: Theory \& Applications. Birkhäuser Boston, 1992. ISBN 1584883553.

C. Eckart. The thermodynamics of irreversible processes. iv. the theory of elasticity and anelasticity. Physical Review, 73(4):373, 1948.

E. Efrati, E. Sharon, and R. Kupferman. Elastic theory of unconstrained non-euclidean plates. Journal of the Mechanics and Physics of Solids, 57(4):762-775, 2009.

J. L. Ericksen and C. Truesdell. Exact theory of stress and strain in rods and shells. Archive for Rational Mechanics and Analysis, 1(1):295-323, 1958.

D. Fox, A. Raoult, and J. Simo. A justification of nonlinear properly invariant plate theories. Archive for rational mechanics and analysis, 124(2):157-199, 1993.

G. Friesecke, R. D. James, and S. Müller. A theorem on geometric rigidity and the derivation of nonlinear plate theory from three-dimensional elasticity. Communications on Pure and Applied Mathematics, 55(11): 1461-1506, 2002a.

G. Friesecke, S. Müller, and R. D. James. Rigorous derivation of nonlinear plate theory and geometric rigidity. Comptes Rendus Mathematique, 334(2):173-178, 2002b.

G. Friesecke, R. D. James, M. G. Mora, and S. Müller. Derivation of nonlinear bending theory for shells from three-dimensional nonlinear elasticity by gamma-convergence. Comptes Rendus Mathematique, 336(8): 697-702, 2003.

Y. Fung. On the foundations of biomechanics. Journal of Applied Mechanics, 50(4b):1003-1009, 1983.

Y. Fung. What are the residual stresses doing in our blood vessels? Annals of biomedical engineering, 19(3): 237-249, 1991.

Y.-C. Fung. Stress, strain, growth, and remodeling of living organisms. In Theoretical, Experimental, and Numerical Contributions to the Mechanics of Fluids and Solids, pages 469-482. Springer, 1995.

L. Fusi, A. Farina, and D. Ambrosi. Mathematical modeling of a solid-liquid mixture with mass exchange between constituents. Mathematics and mechanics of solids, 11(6):575-595, 2006.

A. Geitmann and J. K. Ortega. Mechanics and modeling of plant cell growth. Trends in plant science, 14(9): 467-478, 2009.

S. Germain. Recherches sur la théorie des surfaces élastiques. Mme. Ve. Courcier, Paris, 1821.

A. Goriely and M. Ben Amar. Differential growth and instability in elastic shells. Physical review letters, 94 (19):198103, 2005.

A. Green and W. Zerna. The equilibrium of thin elastic shells. The Quarterly Journal of Mechanics and Applied Mathematics, 3(1):9-22, 1950.

H. Han and Y. Fung. Residual strains in porcine and canine trachea. Journal of biomechanics, 24(5):307-315, 1991. 
G. Helmlinger, P. A. Netti, H. C. Lichtenbeld, R. J. Melder, and R. K. Jain. Solid stress inhibits the growth of multicellular tumor spheroids. Nature biotechnology, 15(8):778-783, 1997.

N. J. Hicks. Notes on Differential Geometry. Van Nostrand mathematical studies, no.3. Van Nostrand Reinhold Co., 1965. ISBN 9780442034108.

K. Hori, M. Suzuki, I. Abe, and S. Saito. Increased tumor tissue pressure in association with the growth of rat tumors. Japanese journal of cancer research: Gann, 77(1):65-73, 1986.

F.-H. Hsu. The influences of mechanical loads on the form of a growing elastic body. Journal of biomechanics, 1(4):303-311, 1968.

J. Humphrey. Cardiovascular Solid Mechanics: Cells, Tissues, and Organs. Springer, 2002. ISBN 9780387951683.

T. A. Ivey and J. M. Landsberg. Cartan for Beginners: Differential Geometry via Moving Frames and Exterior Differential Systems. American Mathematical Society, Providence, RI, 2003.

N. Kadianakis and F. Travlopanos. Kinematics of hypersurfaces in riemannian manifolds. Journal of Elasticity, 111(2):223-243, 2013.

G. Kirchhoff. Über das gleichgewicht und die bewegung einer elastischen scheibe. Journal für die reine und angewandte Mathematik, 40:51-88, 1850.

W. T. Koiter. On the nonlinear theory of thin elastic shells. I- Introductory sections. II- Basic shell equations. III- Simplified shell equations. Koninklijke Nederlandse Akademie van Wetenschappen, Proceedings, Series $B, 69(1): 1-54,1966$.

V. Kondaurov and L. Nikitin. Finite strains of viscoelastic muscle tissue. Journal of Applied Mathematics and Mechanics, 51(3):346-353, 1987.

E. Kröner. Allgemeine kontinuumstheorie der versetzungen und eigenspannungen. Archive for Rational Mechanics and Analysis, 4(1):273-334, 1959.

H. Le Dret and A. Raoult. Le modèle de membrane non linéaire comme limite variationnelle de l'élasticité non linéaire tridimensionnelle. Comptes rendus de l'Académie des sciences. Série 1, Mathématique, 317(2): 221-226, 1993.

V. Lods and B. Miara. Analyse asymptotique des coques en flexion non linéairement élastiques. Comptes rendus de l'Académie des sciences. Série 1, Mathématique, 321(8):1097-1102, 1995.

V. Lods and B. Miara. Nonlinearly elastic shell models: A formal asymptotic approach ii. the flexural model. Archive for rational mechanics and analysis, 142(4):355-374, 1998.

A. E. H. Love. The small free vibrations and deformation of a thin elastic shell. Philosophical Transactions of the Royal Society of London. A, 179:491-546, 1888.

A. E. H. Love. A Treatise on the Mathematical Theory of Elasticity. Cambridge University Press, 1892.

J. Lu and P. Papadopoulos. A covariant constitutive description of anisotropic non-linear elasticity. 51(2): 204-217, 2000. ISSN 0044-2275.

V. Lubarda and A. Hoger. On the mechanics of solids with a growing mass. International journal of solids and structures, 39(18):4627-4664, 2002.

V. A. Lubarda. Constitutive theories based on the multiplicative decomposition of deformation gradient: Thermoelasticity, elastoplasticity, and biomechanics. Applied Mechanics Reviews, 57(2):95-108, 2004.

J. Marsden and T. Hughes. Mathematical Foundations of Elasticity. Dover Civil and Mechanical Engineering Series. Dover, 1983. ISBN 9780486678658. 
J. E. Marsden and T. Ratiu. Introduction to Mechanics and Symmetry. Springer-Verlag, New York, 1994.

E. Mathieu. Mémoire sur le mouvement vibratoire des cloches. 51:177-247, 1882.

J. McMahon, A. Goriely, and M. Tabor. Nonlinear morphoelastic plates I: Genesis of residual stress. Mathematics and Mechanics of Solids, 16(8):812-832, 2011a.

J. McMahon, A. Goriely, and M. Tabor. Nonlinear morphoelastic plates II: Exodus to buckled states. Mathematics and Mechanics of Solids, 16(8):833-871, 2011b.

B. Miara. Nonlinearly elastic shell models: A formal asymptotic approach i. the membrane model. Archive for rational mechanics and analysis, 142(4):331-353, 1998.

P. Naghdi. Foundations of elastic shell theory. In I. N. Sneddon and K. Hill, editors, Progress in Solid Mechanics, volume 4, pages 1-90. North Hollande Publishing Cy, 1963.

S. Nishikawa. Variational Problems in Geometry, volume 205 of Iwanami series in modern mathematics. American Mathematical Society, 2002. ISBN 9780821813560.

V. Novozhilov. The theory of thin shells [translated by P. G. Lowe from the 1951 Russian edition]. P. Noordhoff, 1964.

T. Olsson and A. Klarbring. Residual stresses in soft tissue as a consequence of growth and remodeling: application to an arterial geometry. European Journal of Mechanics-A/Solids, 27(6):959-974, 2008.

J. H. Omens and Y.-C. Fung. Residual strain in rat left ventricle. Circulation Research, 66(1):37-45, 1990.

A. Ozakin and A. Yavari. A geometric theory of thermal stresses. Journal of Mathematical Physics, 51:032902, 2010.

M. Pezzulla, S. A. Shillig, P. Nardinocchi, and D. P. Holmes. Morphing of geometric composites via residual swelling. Soft Matter, 11:5812-5820, 2015a.

M. Pezzulla, G. P. Smith, P. Nardinocchi, and D. P. Holmes. Geometry and mechanics of thin growing bilayers. arXiv:1509.05259v2, pages 1-5, 2015b.

J. B. Pollack, O. Hubickyj, P. Bodenheimer, J. J. Lissauer, M. Podolak, and Y. Greenzweig. Formation of the giant planets by concurrent accretion of solids and gas. Icarus, 124(1):62-85, 1996.

A. Polyanin and V. Zaitsev. Handbook of Nonlinear Partial Differential Equations. CRC Press, 2004. ISBN 9780203489659 .

E. K. Rodriguez, A. Hoger, and A. D. McCulloch. Stress-dependent finite growth in soft elastic tissues. Journal of Biomechanics, 27(4):455-467, 1994.

S. Sadik and A. Yavari. Geometric nonlinear thermoelasticity and the time evolution of thermal stresses. Mathematics and Mechanics of Solids, (DOI: 10.1177/1081286515599458), 2015.

S. Sadik and A. Yavari. On the origins of the idea of the multiplicative decomposition of the deformation gradient. Mathematics and Mechanics of Solids, page DOI:10.1177/1081286515612280, 2016.

J. L. Sanders Jr. Nonlinear theories for thin shells. Technical Report Technical Report No. 10, DTIC Document, 1961.

J. S. Silberberg, P. E. Barre, S. S. Prichard, and A. D. Sniderman. Impact of left ventricular hypertrophy on survival in end-stage renal disease. Kidney Int, 36(2):286-290, 1989.

R. Skalak. Growth as a finite displacement field. In Proceedings of the IUTAM Symposium on Finite Elasticity, pages 347-355. Springer, 1982.

R. Skalak, G. Dasgupta, M. Moss, E. Otten, P. Dullemeijer, and H. Vilmann. Analytical description of growth. Journal of Theoretical Biology, 94(3):555-577, 1982. 
R. Skalak, S. Zargaryan, R. K. Jain, P. A. Netti, and A. Hoger. Compatibility and the genesis of residual stress by volumetric growth. Journal of mathematical biology, 34(8):889-914, 1996.

R. Stojanović, S. Djurić, and L. Vujošević. On finite thermal deformations. Archiwum Mechaniki Stosowanej, 1(16):103-108, 1964.

J. L. Synge and W. Z. Chien. The intrinsic theory of elastic shells and plates. In von Kármán anniv. vol., pages 103-120. Cal. Inst. Tech., Pasadena, 1941.

L. A. Taber. Biomechanics of growth, remodeling, and morphogenesis. Applied mechanics reviews, 48(8): 487-545, 1995.

K. Takamizawa and T. Matsuda. Kinematics for bodies undergoing residual stress and its applications to the left ventricle. Journal of applied mechanics, 57(2):321-329, 1990.

S. Verpoort. The geometry of the second fundamental form: curvature properties and variational aspects. $\mathrm{PhD}$ thesis, Katholieke Universiteit Leuven, 2008.

A. Yavari. A geometric theory of growth mechanics. Journal of Nonlinear Science, 20(6):781-830, 2010.

A. Yavari and A. Goriely. Riemann-Cartan geometry of nonlinear dislocation mechanics. Archive for Rational Mechanics and Analysis, 205(1):59-118, 2012a.

A. Yavari and A. Goriely. Weyl geometry and the nonlinear mechanics of distributed point defects. Proceedings of the Royal Society A, 468:3902-3922, 2012b.

A. Yavari and A. Goriely. Nonlinear elastic inclusions in isotropic solids. Proceedings of the Royal Society A: Mathematical, Physical and Engineering Science, 469(2160):20130415, 2013a.

A. Yavari and A. Goriely. Riemann-Cartan geometry of nonlinear disclination mechanics. Mathematics and Mechanics of Solids, 18(1):91-102, 2013b.

A. Yavari and A. Goriely. The geometry of discombinations and its applications to semi-inverse problems in anelasticity. Proceedings of the Royal Society A, 470:20140403, 2014.

A. Yavari and A. Goriely. The twist-fit problem: Finite torsional and shear eigenstrains in nonlinear elastic solids. Proceedings of the Royal Society A, 471:20150596, 2015.

A. Yavari, J. E. Marsden, and M. Ortiz. On spatial and material covariant balance laws in elasticity. Journal of Mathematical Physics, 47:042903, 2006.

\section{Appendix. Derivation of the Euler-Lagrange equations}

In this appendix, we work out in detail the derivation of the Euler-Lagrange equations first assuming that $\delta \boldsymbol{G}=\delta \boldsymbol{B}=\mathbf{0}$. We substitute (4.8), (4.9), (4.10), (4.11), and (4.12) into (4.7) to obtain

$$
\begin{gathered}
\int_{t_{0}}^{t_{1}} \int_{\mathcal{H}}\left[\frac{\partial \mathcal{L}}{\partial \varphi^{a}} \delta \varphi^{a}+\frac{\partial \mathcal{L}}{\partial \varphi^{n}} \delta \varphi^{n}-\left(\frac{\partial \mathcal{L}}{\partial \mathcal{N}}\right)_{b}\left(\delta \varphi^{a} \beta^{b}{ }_{a}+\frac{\partial\left(\delta \varphi^{n}\right)}{\partial x^{a}} g^{a b}\right)+\frac{\partial \mathcal{L}}{\partial \dot{\varphi}} \cdot \frac{D \delta \varphi}{d t}\right. \\
+\frac{\partial \mathcal{L}}{\partial C_{A B}}\left(2 F^{b}{ }_{B} g_{b c} \delta \varphi^{c}{ }_{\mid A}-2 \delta \varphi^{n} F^{a}{ }_{A} F^{b}{ }_{B} \beta_{a b}\right)+\frac{\partial \mathcal{L}}{\partial \Theta_{A B}}\left(F^{a}{ }_{A} F^{b}{ }_{B} \beta_{a b \mid c} \delta \varphi^{c}+2 F^{a}{ }_{A} \beta_{a c} \delta \varphi^{c}{ }_{\mid B}\right. \\
\left.\left.-\delta \varphi^{n} F^{a}{ }_{A} F^{b}{ }_{B} \beta_{a c} \beta_{b d} g^{c d}+F^{b}{ }_{A}\left(\frac{\partial \delta \varphi^{n}}{\partial x^{b}}\right)_{\mid B}\right)\right] \sqrt{\operatorname{det} \boldsymbol{G}} d X^{I} d t=0 .
\end{gathered}
$$


(.1) yields

$$
\begin{gathered}
\int_{t_{0}}^{t_{1}} \int_{\mathcal{H}}\left\{\frac{\partial \mathcal{L}}{\partial \varphi^{a}} \delta \varphi^{a}+\frac{\partial \mathcal{L}}{\partial \varphi^{n}} \delta \varphi^{n}+\frac{1}{\sqrt{\operatorname{det} \boldsymbol{G}}} \frac{d}{d t}\left(\sqrt{\operatorname{det} \boldsymbol{G}} \frac{\partial \mathcal{L}}{\partial \dot{\varphi}} \cdot \delta \varphi\right)-\frac{1}{\sqrt{\operatorname{det} \boldsymbol{G}}} \frac{D}{d t}\left(\sqrt{\operatorname{det} \boldsymbol{G}} \frac{\partial \mathcal{L}}{\partial \dot{\varphi}}\right) . \delta \varphi\right. \\
-\left(\frac{\partial \mathcal{L}}{\partial \mathcal{N}}\right)_{b} \beta^{b}{ }_{a} \delta \varphi^{a}-\left(\left(\frac{\partial \mathcal{L}}{\partial \mathcal{N}}\right)_{b} \delta \varphi^{n} g^{a b} F^{-A}\right)_{a}+\left(\left(\frac{\partial \mathcal{L}}{\partial \mathcal{N}}\right)_{b} g^{a b} F^{-A}{ }_{a}\right)_{\mid A} \delta \varphi^{n} \\
+2\left(\frac{\partial \mathcal{L}}{\partial C_{A B}} F^{c}{ }_{A} g_{a c} \delta \varphi^{a}\right)_{\mid B}-2\left(\frac{\partial \mathcal{L}}{\partial C_{A B}} F^{c}{ }_{A} g_{a c}\right)_{\mid B} \delta \varphi^{a}-2 \frac{\partial \mathcal{L}}{\partial C_{A B}} F^{a}{ }_{A} F^{b}{ }_{B} \beta_{a b} \delta \varphi^{n} \\
+\frac{\partial \mathcal{L}}{\partial \Theta_{A B}} F^{c}{ }_{A} F^{b}{ }_{B} \beta_{b c \mid a} \delta \varphi^{a}+2\left(\frac{\partial \mathcal{L}}{\partial \Theta_{A B}} F^{c}{ }_{A} \beta_{a c} \delta \varphi^{a}\right)_{\mid B}-2\left(\frac{\partial \mathcal{L}}{\partial \Theta_{A B}} F^{c}{ }_{A} \beta_{a c}\right)_{\mid B} \delta \varphi^{a} \\
-\frac{\partial \mathcal{L}}{\partial \Theta_{A B}} F^{a}{ }_{A} F^{b}{ }_{B} \beta_{a c} \beta_{b d} g^{c d} \delta \varphi^{n}+\left(\frac{\partial \mathcal{L}}{\partial \Theta_{A B}} F^{b}{ }_{A} \frac{\partial \delta \varphi^{n}}{\partial x^{b}}\right)_{\mid B}-\left[\left(\frac{\partial \mathcal{L}}{\partial \Theta_{A B}} F^{b}{ }_{A}\right)_{\mid B} \delta \varphi^{n} F^{-D_{b}}\right]_{\mid D} \\
\left.+\left[\left(\frac{\partial \mathcal{L}}{\partial \Theta_{A B}} F^{b}{ }_{A}\right)_{\mid B} F^{-D}{ }_{b}\right]_{\mid D} \delta \varphi^{n}\right\}_{\sqrt{\operatorname{det} \boldsymbol{G}} d X^{I} d t=0 .}
\end{gathered}
$$

We can rewrite (.2) as

$$
\begin{aligned}
& \int_{t_{0}}^{t_{1}} \int_{\mathcal{H}}\left\{\frac{1}{\sqrt{\operatorname{det} \boldsymbol{G}}} \frac{d}{d t}\left(\sqrt{\operatorname{det} \boldsymbol{G}} \frac{\partial \mathcal{L}}{\partial \dot{\varphi}} \cdot \delta \varphi\right)+2\left(\frac{\partial \mathcal{L}}{\partial C_{A B}} F^{c}{ }_{A} g_{a c} \delta \varphi^{a}\right)_{\mid B}+2\left(\frac{\partial \mathcal{L}}{\partial \Theta_{A B}} F^{c}{ }_{A} \beta_{a c} \delta \varphi^{a}\right)_{\mid B}\right. \\
& -\left[\left(\frac{\partial \mathcal{L}}{\partial \Theta_{A B}} F_{A}^{b}\right)_{\mid B} \delta \varphi^{n} F^{-D}\right]_{\mid D}-\left(\left(\frac{\partial \mathcal{L}}{\partial \mathcal{N}}\right)_{b} \delta \varphi^{n} g^{a b} F^{-A}{ }_{a}\right)_{\mid A}+\left(\frac{\partial \mathcal{L}}{\partial \Theta_{A B}} \frac{\partial \delta \varphi^{n}}{\partial X^{A}}\right)_{\mid B} \\
& +\left[\frac{\partial \mathcal{L}}{\partial \varphi^{a}}-\left(\frac{\partial \mathcal{L}}{\partial \mathcal{N}}\right)_{b} \beta^{b}{ }_{a}-\frac{1}{\sqrt{\operatorname{det} \boldsymbol{G}}} \frac{D}{d t}\left(\sqrt{\operatorname{det} \boldsymbol{G}} \frac{\partial \mathcal{L}}{\partial \dot{\varphi}^{a}}\right)-2\left(\frac{\partial \mathcal{L}}{\partial C_{A B}} F^{c}{ }_{A} g_{a c}\right)_{\mid B}\right. \\
& \left.+\frac{\partial \mathcal{L}}{\partial \Theta_{A B}} F_{A}^{c} F^{b}{ }_{B} \beta_{b c \mid a}-2\left(\frac{\partial \mathcal{L}}{\partial \Theta_{A B}} F^{c}{ }_{A} \beta_{a c}\right)_{\mid B}\right] \delta \varphi^{a}+\left[\frac{\partial \mathcal{L}}{\partial \varphi^{n}}+\left(\left(\frac{\partial \mathcal{L}}{\partial \mathcal{N}}\right)_{b} g^{a b} F^{-A}{ }_{a}\right)_{\mid A}\right. \\
& -\frac{1}{\sqrt{\operatorname{det} \boldsymbol{G}}} \frac{D}{d t}\left(\sqrt{\operatorname{det} \boldsymbol{G}} \frac{\partial \mathcal{L}}{\partial \dot{\varphi}^{n}}\right)-2 \frac{\partial \mathcal{L}}{\partial C_{A B}} F^{a}{ }_{A} F^{b}{ }_{B} \beta_{a b}-\frac{\partial \mathcal{L}}{\partial \Theta_{A B}} F^{a}{ }_{A} F^{b}{ }_{B} \beta_{a c} \beta_{b d} g^{c d} \\
& \left.\left.+\left[\left(\frac{\partial \mathcal{L}}{\partial \Theta_{A B}} F_{A}^{b}\right)_{\mid B} F^{-D}\right]_{\mid D}\right]_{\delta}^{n}\right\} d S d t=0 .
\end{aligned}
$$

At $t=t_{1}$, we assume that $\varphi_{\epsilon, t_{1}}=\varphi_{t_{1}}$ so that $\delta \varphi_{t_{1}}=0$. Therefore, by integrating the first term in (.3) in the time domain, we obtain only one term at $t=t_{0}$ giving the initial condition on the velocity at $t=t_{0}$. By applying Stokes' theorem to the following five terms, if we denote by $\mathbf{T}$ the outward in-plane vector field normal to the boundary curve $\partial \mathcal{H}$, we obtain

$$
\begin{gathered}
-\left.\int_{\mathcal{H}} \frac{\partial \mathcal{L}}{\partial \dot{\varphi}} \cdot \delta \varphi d S\right|_{t=t_{0}}+\int_{t_{0}}^{t_{1}} \int_{\partial \mathcal{H}}\left\{\left(2 \frac{\partial \mathcal{L}}{\partial C_{A B}} F^{c}{ }_{A} g_{a c}+2 \frac{\partial \mathcal{L}}{\partial \Theta_{A B}} F^{c}{ }_{A} \beta_{a c}\right) \mathrm{T}_{B} \delta \varphi^{a}\right. \\
\left.-\left[\left(\frac{\partial \mathcal{L}}{\partial \Theta_{A C}} F^{b}{ }_{A}\right)_{\mid C} F^{-B}{ }_{b}+\left(\frac{\partial \mathcal{L}}{\partial \mathcal{N}}\right)_{b} g^{a b} F^{-B}{ }_{a}\right] \mathrm{T}_{B} \delta \varphi^{n}+\frac{\partial \mathcal{L}}{\partial \Theta_{A B}} \mathrm{~T}_{B} \frac{\partial \delta \varphi^{n}}{\partial X^{A}}\right\} d L d t \\
\int_{t_{0}}^{t_{1}} \int_{\mathcal{H}}\left\{\left[\frac{\partial \mathcal{L}}{\partial \varphi^{a}}-\left(\frac{\partial \mathcal{L}}{\partial \mathcal{N}}\right)_{b} \beta^{b}{ }_{a}-\frac{1}{\sqrt{\operatorname{det} \boldsymbol{G}}} \frac{D}{d t}\left(\sqrt{\operatorname{det} \boldsymbol{G}} \frac{\partial \mathcal{L}}{\partial \dot{\varphi}^{a}}\right)-2\left(\frac{\partial \mathcal{L}}{\partial C_{A B}} F^{c}{ }_{A} g_{a c}\right)_{\mid B}\right.\right. \\
\left.+\frac{\partial \mathcal{L}}{\partial \Theta_{A B}} F_{A}^{c} F_{B}^{b}{ }_{B} \beta_{b c \mid a}-2\left(\frac{\partial \mathcal{L}}{\partial \Theta_{A B}} F^{c}{ }_{A} \beta_{a c}\right)_{\mid B}\right] \delta \varphi^{a}+\left[\frac{\partial \mathcal{L}}{\partial \varphi^{n}}+\left(\left(\frac{\partial \mathcal{L}}{\partial \mathcal{N}}\right)_{b} g^{a b} F^{-A_{a}}\right)_{\mid A}\right. \\
-\frac{1}{\sqrt{\operatorname{det} \boldsymbol{G}} \frac{D}{d t}}\left(\sqrt{\operatorname{det} \boldsymbol{G}} \frac{\partial \mathcal{L}}{\partial \dot{\varphi}^{n}}\right)-2 \frac{\partial \mathcal{L}}{\partial C_{A B}} F^{c}{ }_{A} F^{b}{ }_{B} \beta_{b c}-\frac{\partial \mathcal{L}}{\partial \Theta_{A B}} F^{a}{ }_{A} F^{b}{ }_{B} \beta_{a c} \beta_{b d} g^{c d} \\
\left.\left.+\left[\left(\frac{\partial \mathcal{L}}{\partial \Theta_{A B}} F^{b}{ }_{A}\right)_{\mid B} F^{-D}{ }_{b}\right]_{\mid D}\right] \delta \varphi^{n}\right\} d S d t=0 .
\end{gathered}
$$


By arbitrariness of $\delta \varphi^{\|}, \delta \varphi^{n}$, and $d\left(\delta \varphi^{n}\right)$, the Euler-Lagrange equations for shells (4.13) together with the initial and boundary conditions (4.14) follow from (.4). Note that following Codazzi's equation (2.3), we have $\beta_{b c \mid a}=\beta_{a c \mid b}$. Therefore $\frac{\partial \mathcal{L}}{\partial \Theta_{A B}} F^{c}{ }_{A} F^{b}{ }_{B} \beta_{b c \mid a}=\frac{\partial \mathcal{L}}{\partial \Theta_{A B}} F^{c}{ }_{A} \beta_{a c \mid B}$. 